\title{
23rd annual SASRO meeting
}

Congress president: Prof. Jean Bourhis, MD, PhD

SwissTech Convention Center, Route Louis-Favre 2, 1024 Ecublens

\section{Scientific committees}

\author{
Scientific committee-medical physicists \\ Nathan Corradini \\ Michael Fix \\ Maud Jaccard \\ Raphael Möckli \\ Stephanie Tanadini-Lang
}
Scientific committee-technicians
Leonie Heym
Frederic Duclos
Helen Summers
Christoph Winter

\author{
Scientific committee-biologists \\ Martin Pruschy \\ Marie Catherine Vozenin \\ Scientific committee-clinical \\ Jean Bourhis \\ Matthias Guckenberger \\ Oscar Matzinger \\ Oliver Riesterer \\ Pelagia Tsoutsou \\ Frank Zimmermann
}




\section{Type: Physics}

\section{Correlation between 2D and 3D patient specific quality assurance in head and neck cancers \\ Type: Physics}

\section{Presenting Author:}

Suman Bhasker

\section{Institutions:}

All India institute of Medical Sciences, New Delhi

Department:

Radiation Oncology

Aims: To find correlation between 2D-Gamma passing rate and 3D-dose volume histogram (DVH) metrics for pre-treatment patient-specific quality-assurance (QA).

Methods and materials: 25 head and neck patients, treated with VMAT were selected and planned with Elekta Versa HD linear-accelerator using Monaco (5.11) treatment planning system. 2D-planar dose measurements were done with IBA-I'matriXX evolution detector-array using My-QA-Patients software. For 2D-Gamma index evaluation $3 \% / 3 \mathrm{~mm}$ and $2 \% / 2 \mathrm{~mm}$ criteria was used. 3D-dose measurements were done using IBA-COMPASS system. For 3D-measurement, Monaco and COMPASS doses were compared by means of Percentage (\%age) dose differences to PTV and organ at risks (OAR). For PTV $D_{95}, D_{2}$ and $D_{50}$ (dose received by $95 \%, 2 \%$ and $50 \%$ volume), similarly for OARs (spine and brainstem) $\mathrm{D}_{2}$ was noted. Correlation coefficient with corresponding two tailed $p$-value $(\leq 0.05$, for statistically-significant) was calculated for 2D-gamma passing rate and \%age dose differences of 3D-DVH based metrics (Monaco calculated versus COMPASS measured). Correlation will be considered weak or strong based on $r$ value $\leq 0.59$ and $\geq 0.60$ respectively.

Results: 2D-Gamma index (global) passing-rate was $98.2 \pm 1.6 \%$, $92.1 \pm 5.5 \%$ for $3 \% / 3 \mathrm{~mm}$ and $2 \% / 2 \mathrm{~mm}$ criteria. \%age dose-differences for PTV D95, D2, D50 were: $3.22 \pm 2.09 \%, 3.65 \pm 3.23 \%, 2.93 \pm 1.59$ respectively. For Spine and brainstem D2 were as: $-1.64 \pm 5.10 \%$, $1.77 \pm 2.45 \%$.

Correlation coefficient between PTV DVH based indices and 2D-gamma $(3 \% / 3 \mathrm{~mm})$ were: PTV-D95 $(\mathrm{r}=0.356, p=0.112)$, PTV-D2 $(\mathrm{r}=0.278, p=0.223)$ and PTV-D50 $(\mathrm{r}=0.305, p=0.178)$. For spine and brainstem D2 (\% dose difference) with 2D-gamma (3\%/3 mm) showed Correlation coefficient $(\mathrm{r}=0.469, p=0.077),(\mathrm{r}=0.373, p=0.154)$ respectively.

Correlation analysis for 3D DVH based metrics and 2D-gamma $(2 \% / 2 \mathrm{~mm})$ were as: PTV-D95( $\mathrm{r}=0.529, p=0.138)$, PTV-D2 $(\mathrm{r}=0.462, p=0.054)$ and PTV-D50 $(\mathrm{r}=0.468, p=0.351)$. For spine and brainstem D2 with $2 \mathrm{D}$-gamma $(2 \% / 2 \mathrm{~mm})$ showed Correlation coefficient $(\mathrm{r}=0.202, p=0.406),(\mathrm{r}=0.331, p=0.143)$ respectively.

Conclusions: Weak correlation (not statistically significant) was seen between 2D-Gamma-index passing rate and 3D-DVH based pre-treatment QA using COMPASS.

\section{Evaluation of patient specific quality assurance using COMPASS for head and neck cancer}

Type: Physics

\section{Presenting Author:}

Subhash Chander

\section{Institutions:}

All India institute of Medical Sciences, New Delhi

Department:

Radiation Oncology

Aim: To evaluate 3D dose-volume-histogram (DVH) based pre-treatment plan verification results using COMPASS, in addition to 2D-planar quality assurance.
Methods: $25 \mathrm{HNC}$ cases treated with volumetric modulated arc therapy were planned with 6MV X-rays on Elekta Versa HD linear-accelerator. Prescription dose ranged from 1.8 to $3.0 \mathrm{~Gy}$ per-fraction. Treatment planning was done using Elekta Monaco (5.11) system. 2D-planar dose measurements were done with IBA-I'matriXX evolution detector array using My-QA-Patients software. For 2D-Gamma index evaluation $3 \% / 2 \mathrm{~mm}, 10 \%$ threshold criteria was used. 3D-dose measurements were done using IBA-COMPASS software along with I'matriXX evolution detector. Monaco calculated and COMPASS (measured and calculated) doses were compared using DVH, in terms of doses to planning target volume (PTV) and organ at risks (OAR). For PTV $\mathrm{D}_{95}, \mathrm{D}_{2}$ and $\mathrm{D}_{50}$ (dose received by $95 \%, 2 \%$ and $50 \%$ volume), similarly for OARs $\mathrm{D}_{2}$ and $\mathrm{D}_{50}$ was noted.

Results: Monaco v/s COMPASS computed variations (cGy) were as: $\mathrm{PTVD}_{95}(-63.62 \pm 153.30 \mathrm{cGy}), \mathrm{PTVD}_{2}(63.24 \pm 105.68 \mathrm{cGy})$, $\mathrm{PTVD}_{50}(-42.13 \pm 85.97 \mathrm{cGy})$ respectively. Rt. $-\mathrm{EyeD}_{2}(-78.35 \pm 121.83)$, $\mathrm{D}_{50}(56.08 \pm 92.43 \mathrm{cGy})$; Lt. $-\mathrm{EyeD}_{2}(-72.70 \pm 111.72 \mathrm{cGy}), \mathrm{D}_{50}(-37.43 \pm$ 94.21cGy); Rt.-Optic-nerveD $(-143.44 \pm 147.83 \mathrm{cGy}), \mathrm{D}_{50}(-46.94 \pm$ $119.34 \mathrm{cGy})$; Lt.-Optic-nerveD $\mathrm{D}_{2}(-127.50 \pm 188.76 \mathrm{cGy}), \mathrm{D}_{50}(-54.60 \pm$ $136.45 \mathrm{cGy}) ; \quad$ BrainstemD $\mathrm{D}_{2}(-53.67 \pm 142.43 \mathrm{cGy}), \quad \mathrm{D}_{50}(-44.81 \pm$ $63.14 \mathrm{cGy})$; Spinal-cordD $2(28.97 \pm 55.56 \mathrm{cGy}), \mathrm{D}_{50}(12.36 \pm 37.74 \mathrm{cGy})$.

Monaco computed v/s COMPASS measured variations (cGy) were as: $\operatorname{PTVD}_{95}(239.44 \pm 128.32 \mathrm{cGy}), \quad \mathrm{PTVD}_{2}(225.04 \pm$ $119.43 \mathrm{cGy}), \quad \mathrm{PTVD}_{50}(216.17 \pm 99.87 \mathrm{cGy})$ respectively. Rt.-EyeD (112.78 \pm 249.47$), \quad \mathrm{D}_{50}(130.27 \pm 134.87 \mathrm{cGy}) ; \quad$ Lt.-EyeD $(175.46 \pm 244.38 \mathrm{cGy}), \quad \mathrm{D}_{50}(169.46 \pm 174.19 \mathrm{cGy}) ; \quad$ Rt.-Optic-nerveD $\mathrm{D}_{2}$ (147.81 $\pm 171.62 \mathrm{cGy}), \quad \mathrm{D}_{50}(96.90 \pm 272.54 \mathrm{cGy}) ; \quad$ Lt.-Optic-nerve $\mathrm{D}_{2}$ $(66.93 \pm 221.30 \mathrm{cGy}), \quad \mathrm{D}_{50}(114.72 \pm 274.08 \mathrm{cGy}) ;$ BrainstemD $_{2}(58.80 \pm$ $117.41 \mathrm{cGy}), \mathrm{D}_{50}(9.77 \pm 115.84 \mathrm{cGy})$; Spinal-cordD $2(33.45 \pm 123.46 \mathrm{cGy})$, $\mathrm{D}_{50}(-6.10 \pm 125.78 \mathrm{cGy})$.

Conclusions: $2 \mathrm{D}-$ Gamma pass-rate was $97.4 \pm 2.3 \%$ for $3 \% / 2 \mathrm{~mm}$ criteria. COMPASS calculated and measured doses showed good agreement $(<2.5 \mathrm{~Gy})$ with Monaco calculated dose, hence can be used for pre-treatment QA.

\section{Impact of electromagnetic stray radiation in the hyperthermia treatment room \\ Type: Physics}

Presenting Author:

Dietmar Marder

Institutions:

Kantonsspital Aarau, Kantonsspital Baden

Department:

Radio-Onkologie-Zentrum

Aims: Hyperthermia to temperatures of $41-43^{\circ} \mathrm{C}$ has been shown to be a valuable sensitizer for radiation- and chemo-therapy in cancer treatment. During hyperthermia treatment $(\mathrm{HT})$ the presence of health staff and accompanying persons in the treatment room is desirable for patient comfort. In this study the electromagnetic stray radiation in the treatment room with respect to limits for non-ionizing radiation was investigated.

Methods: The strength of the electromagnetic field inside the unoccupied HT room was measured with a calibrated power density meter (RAHAM Model 4C, General Microwave Corporation Amityville (NY), USA). Measurements were made on a 2-dimensional grid with measurement point $20 \mathrm{~cm}$ apart. During the measurements the HT machine (BSD2000-3D with SigmaEye applicator, Pyrexar Medical, Salt Lake City, USA) was running with a dummy load of saline water. Power at the generator output was $1000 \mathrm{~W}$ maximum, distributed through the feeding network to 24 antennas of the SigmaEye applicator. The results of the electrical field strength measurements were evaluated according to the ICNIRP guidelines [1].

Results: A clear linear relation between output power at the generator and measured power density at a single measurement point was found. 
Inside the HT room electrical field strength values between $17.4 \mathrm{~V} / \mathrm{m}$ and $137.3 \mathrm{~V} / \mathrm{m}$ were detected. According to the ICNIRP guidelines [1] areas with electrical field strength below and above the limit for occupational exposure of $61 \mathrm{~V} / \mathrm{m}$ were detected and marked on the floorplan of the HT room for a typical patient treatment generator output power of $800 \mathrm{~W}$.

Conclusion: In large areas of the empty HT room the limits for occupational exposure are exceeded. Specific areas around the treatment table were identified as safe. Since the presence of persons and objects inside the HT room strongly influences the measured field strength further measurements should investigate the actual field strengths during patient treatment and indicate measures to avoid high E-fields.

\section{References}

1. CNIRP (1998) Guidelines for Limiting Exposure to Time-Varying Electric, Magnetic, and Electromagnetic Fields (up to $300 \mathrm{GHz}$ ). Health Physics 74(4):494-522

\section{Initial clinical experience with the MR-Linac system-Patient perspective \\ Type: Physics}

Presenting Author:

Jonathan Day

Authors:

A. Moreira, J. Day, B. König-Nettelmann, N. Andratschke,

M. Guckenberger, H. I. Garcia Schüler, C. Witt, S. Tanadini-Lang

\section{Institutions:}

University Hospital Zurich

Department:

RadioOnkologie

Objectives: Linear accelerators with integrated MRI have many advantages such as enhanced soft tissue contrast, intrafractional imaging and the possibility to adapt the dose daily. However, disadvantages are lengthened treatment times and the closed environment. This study aimed at evaluating patient experience in this new machine.

Materials and methods: Patients were initially screened for MRI compatibility and claustrophobia by one of the clinicians trained on the MR-Linac. All patients who were referred to the MRIdian ${ }^{\mathrm{TM}}$ (Viewray) for treatment received an in-house developed questionnaire consisting of 15 open-ended questions. This contained questions regarding impressions of the machine/room, the experience of lying in the machine and tolerability of treatment duration and position. 11 patients treated with the MRIdian system were given questionnaires at the time of MR-Simulation, 1st and last treatment to measure patient-reported outcome over time. 21 questionnaires were evaluated.

Results: The length of treatment was acceptable ( $\geq 7$ out of 10) in $94 \%$ of responses but only $76 \%$ gave the same score to comfort of the treatment position. Patients were comfortable with machine noise as well as the feeling of being in a confined space (median 10/10). Of the 6 patients that received breathing instructions, breathing commands had a median score of $9 / 10$ with a range of $6-10$. The largest change in response from a single patient at MR-sim to last treatment was in anxiety (decrease of 3 points) but median change in score across all patients was $0.3 \%$. No large change was noted in other groups. All patients scored at least $9 / 10$ satisfaction with information provided to them before and during their radiation.

Conclusion: After careful patient selection, all simulated patients were able to undergo treatment on the MRIdian System. Treatment was well tolerated and patients scored positively despite the lengthy treatment process of daily adaption and the closed environment.
Dosimetric and geometric verification of elements multimetastases v.2.0, for $6 \mathrm{MV}$ and $6 \mathrm{FFF}$

Type: Physics

Presenting Author:

Sara Alonso Arrizabalaga

Authors:

Alonso S, Lomax N, Rogers S, Schürkens J, Beltz M, Lutters G

Institutions:

Kantonsspital Aarau, Kantonsspital Baden

Department:

Radio-Onkologie-Zentrum

Aims: Elements Multi Metastases (BrainLab Ltd) is a single isocentre multiple metastases stereotactic planning module that has been routinely used in Kantonsspital Aarau (KSA) for the past 2 years with $6 \mathrm{MV}$. Dosimetric and geometric verification of the recently released version 2.0, (which also optimises to allow Organs At Risk (OARs) sparing, offering an alternative to VMAT), is required for both 6MV and 6FFF with our Novalis Truebeam STX linac before clinical implementation. For FFF beams the verification of off axis targets is of particular relevance in single isocentre treatments.

Methods: The dosimetric verification was carried out in an anthropomorphic phantom (Max-HD(IMT)), using a microdiamond chamber to measure at 5 target positions in $6 \mathrm{MV}$ and $6 \mathrm{FFF}$ plans, (10 arcs, single isocenter). The plans were recalculated in Eclipse ${ }^{\mathrm{TM}}$ Treatment Planning System (AAA algorithm), as well as in Mobius (Collapsed Cone Convolution algorithm with manufacturer supplied True Beam Golden Beam Data). Eight hidden target points were verified geometrically in an End to End (E2E) test with films inside Max-HD, using a 10 arc, non-coplanar approach. Distances from the isocenter to the lesions ranged from 27 to $57 \mathrm{~mm}$. For both dosimetric and geometric testing, image guided setup of the phantom was carried out using ExacTrac prior to irradiating each beam.

Results: The measured values and those calculated by Elements v.2.0 show a mean difference of $1.7 \pm 0.7 \%$ for $6 \mathrm{MV}$ and $0.2 \pm 1.8 \%$ for 6 FFF. Eclipse calculations vs. Elements result in a mean difference of $1.0 \pm 1.1 \%$ and $1.1 \pm 1.0 \%$ for $6 \mathrm{MV}$ and $6 \mathrm{FFF}$ respectively, and of $-1.9 \pm 0.4 \%$ and $-1.7 \pm 0.7 \%$ for $6 \mathrm{MV}$ and $6 \mathrm{FFF}$ for Mobius. The E2E test shows a geometric accuracy of $\leq 0.5 \mathrm{~mm}$ for both energies.

Conclusion: Successful validation of Elements v.2.0 should allow both $6 \mathrm{MV}$ and 6FFF (with shorter delivery times) to be used clinically for targets up to $5 \mathrm{~cm}$ off axis, allowing more widespread use due to sparing of OARs.

\section{New approaches for detection of wide energy spectrum of neutrons by nuclear emulsion detectors for medical application}

Type: Physics

Presenting Author:

Dr. phil. nat. Alireza Ehtesham

Institutions:

Bern University

Department:

Albert Einstein Center for Fundamental Physics Laboratory for High Energy Physics

The poster presents a series of experimental achievements for new approaches in the detection and spectrometry of fast and high energy neutrons in the wide range from 5 to $600 \mathrm{MeV}$, by using nuclear emulsion detector films. The sensitivity and detection efficiency response of nuclear emulsion detectors are measured. The study is done to study of neutron detection via recoil proton production in a multi-layer detector structure, likewise the Emulsion Cloud Chamber (ECC). Reconstructing proton micro-tracks obtains the tracks with several centime- 
tres length, which is used to calculate and obtain high energy spectrum of detected neutrons. The emulsion film type is made by Fuji company for the OPERA (Oscillation Project with Emulsion tRacking Apparatus) experiment. For this work, a collaboration between facilities and laboratories has been planned. The cooperation was between the CERF facilities at CERN, one of biggest international facilities in neutron physics research and Laboratory for High Energy Physics (LHEP) in the University of Bern which is the biggest nuclear emulsion laboratory in Europe and the second in the world. The thesis presents achievements using the advantages of nuclear emulsion detector, such as the low price, no power supplies during exposure, directional detection, high resolution and wide angular acceptance, which make this type of detector an interesting choice for neutron detection and spectrometry. To be impervious to the magnetic field during exposure, makes nuclear emulsions suitable to use in accelerator and new combined facilities with applied magnetic fields, such as a new generation of therapeutic linear accelerators with magnetic resonance imaging (MRI) machine. Also these favourable circumstances with ability in directional detection help to detect and identify unreachable or hidden nuclear radiation sources, materials or damaged facilities behind thick shielding.

\section{Clinical evaluation of a novel optimization algorithm for cyberknife IRIS-based treatment plans \\ Type: Physics}

\section{Presenting Author:}

Michele Zeverino

Authors:

M. Zeverino ${ }^{1}$, M. Marguet ${ }^{1}$, C. Zulliger ${ }^{2}$, A. Durham ${ }^{2}$, R. Jumeau ${ }^{2}$, F. Herrera ${ }^{2}$, L. Schiappacasse ${ }^{2}$, J. Bourhis ${ }^{2}$, F. Bochud ${ }^{1}$, R. Moeckli

\section{Institutions:}

IRA, CHUV, Lausanne

Department:

${ }^{1}$ Institute of Radiation Physics, ${ }^{2}$ Department of Radiation Oncology

Purpose: To report on the clinical implementation of the VOLO ${ }^{\mathrm{TM}}$ optimization algorithm for SRS/SBRT treatments and its dosimetric comparison with the previous Sequential Optimization (SO) algorithm.

Materials and methods: Forty patients treated for brain, spine, prostate and lung tumors, and planned with the SO algorithm were re-planned with $\mathrm{VOLO}^{\mathrm{TM}}$. Comparison involved target coverage, conformity, gradient and homogeneity indexes (CN, GI and $\mathrm{HI}$ ) and specific indicators of dose to OARs. Plans were also compared in terms of number of nodes, beams, MU and delivery time. All dose distributions designed with VOLO $^{\mathrm{TM}}$ were validated via ionisation chamber measurements.

Results: The following statistically significant dosimetric differences were observed. VOLO ${ }^{\mathrm{TM}}$ was superior in terms of target coverage for prostate (99\% vs $96 \%$ ) and spine (92\% vs $81 \%$ ), GI for brain $(4.41$ vs 4.76$), \mathrm{CN}$ for brain (0.77 vs 0.72$)$, and for brain and urethra dose sparing. SO gave better results for GI for prostate (3.67 vs 4.05 ) and maximum dose to brain stem. VOLO $^{\mathrm{TM}}$ showed a steeper peripheral dose fall-off for brain and lung cases; SO was superior for prostate and spine cases. Overall, the number of nodes, beams and MU were reduced with $\mathrm{VOLO}^{\mathrm{TM}}$ up to $36 \%, 14 \%$ and $31 \%$, respectively. The average reduction of delivery time was $20 \%$ (ranging from $8 \%$ for brain to $30 \%$ for prostate). The mean 1D dose measurement deviation from calculation was $-0.2 \%$ (range $-1.3-1.7 \%$ ). $2 \mathrm{D}$ dose measurements returned a mean distance-to-agreement of $0.8 \pm 0.8 \mathrm{~mm}$ and a mean dose difference of $0.6 \pm 1.0 \%$.

Conclusions: VOLO ${ }^{\mathrm{TM}}$ optimization algorithm provided clinically acceptable dose distributions, most of the time better than SO. The treatment time was significantly reduced up to $30 \%$. The peripheral dose fall-off increased for large collimator sizes. The verification measurements proved its clinical implementation.
Combined proton-photon radiotherapy using a fixed proton beamline fully integrated into a conventional treatment room Type: Physics

Presenting Author:

Silvia Fabiano

Authors:

S. Fabiano, P. Balermpas, M. Guckenberger, J. Unkelbach

Institutions:

University Hospital Zurich

Department:

Department of Radiation Oncology

Aim: The physical advantages offered by protons over photons are nowadays established. However, Proton Therapy (PT) is still a limited resource, which is not available to all the patients who may benefit from it. We explore the potential of a new cost-effective design for PT, which may facilitate proton treatments in conventional treatment rooms and allow the widespread use of protons.

Methods: We consider the following design. The treatment room consists of a standard Linac for X-ray therapy (IMRT/VMAT), a motorized couch to treat the patient in lying position, and a horizontal proton beamline equipped with pencil beam scanning. With this setup, proton plans may be sub-optimal as beam angles are limited to a coronal plane. However, high-quality treatment plans may be realized by delivering protons and photons in the same fraction. Treatment planning is performed by simultaneously optimizing IMRT and IMPT plans based on their cumulative physical dose. We demonstrate this concept for a nasopharyngeal cancer case. The combined treatment uses 35 fractions to deliver 70/60/54 Gy to GTV/PTV-T/PTV-N and is obtained by combining a 19-beams IMRT plan with a 4-fields IMPT plan.

Results: In the optimal combination, photons are used to improve dose conformity while protons allow reducing the integral dose to normal tissues. In fact, the combined treatment improves on both single-modality IMRT and IMPT plans for the main organs at risk and achieves $79 \%$ of integral dose reduction in the remaining healthy tissues that the IMPT plan yields. The lower doses that can be obtained with combined proton-photon treatments translate into a $10 \%$ and $9 \%$ lower risk for xerostomia and oral mucositis compared to the pure IMRT plan.

Conclusions: Cost-effective PT systems will likely include a fixed beamline rather than a gantry. Proton-photon combinations substantially improve on IMRT/VMAT plans and reduce the risk of side effects while making protons available to more patients.

\section{Quality assurance of a randomized phase II trial on urethra- sparing prostate SBRT \\ Type: Physics}

Presenting Author:

Maud Jaccard

Authors:

M.Jaccard ${ }^{1}$, T. Zilli ${ }^{1}$, A. Dubouloz ${ }^{1}$, L. Tsvang ${ }^{2}$, N. Linthout ${ }^{3}$, W. Verbakel ${ }^{4}$, M. Björkqvist ${ }^{5}$, L. Escude ${ }^{6}$, J. Lencart $^{7}$, Z. Ozen ${ }^{8}$, J. Pérez-Moreno ${ }^{9}$, M. Rouzaud ${ }^{1}$, R. Miralbell ${ }^{1,2}$

\section{Institutions:}

${ }^{1}$ Geneva University Hospital, ${ }^{2}$ Ramat Gan, ${ }^{3}$ Aalst., ${ }^{4}$ Amsterdam, ${ }^{5}$ Turku, ${ }^{6}$ Barcelona, ${ }^{7}$ Porto, ${ }^{8}$ Istanbul, ${ }^{9}$ Madrid

Department:

${ }^{1}$ Radiation Oncology

Aims: To present the radiotherapy quality assurance (RTQA) results from a prospective multicenter phase II randomized trial of short $v s$. protracted urethra-sparing stereotactic body radiotherapy (SBRT) for localized prostate cancer $(\mathrm{PCa})$.

Methods: Between 2012 and 2015, 165 patients (pts) from 9 centers with localized PCa were randomized and treated either in 9 days $(82$ 
pts), or in 28 days ( 83 pts). 36.25 Gy in 5 fractions were prescribed to the prostate PTV, while the urethra planning risk volume (uPRV) received 32.5 Gy. Pts were treated either with VMAT $(n=112)$ or IMRT $(n=53)$. We retrospectively analyzed deviations from protocol dose constraints, PTV homogeneity index (HI), Dice similarity coefficient (DSC) and the number of monitor units (MU) per plan, and compared dosimetric results of VMAT $v s$. IMRT.

Results: There was at least one major protocol deviation for $52 \mathrm{pts}$ in the study (32\%), mainly for uPRV (12\%) and PTV (5\%) coverage, and for the rectal and bladder wall (BW) $\mathrm{V}_{90 \%}(7 \%$ each). Violations were more frequent in the IMRT group for PTV and UPRV coverage ( $p=0.001$ and $p<0.0001$, resp.). Furthermore, the use of a VMAT technique yielded better uPRV coverage $\left(\mathrm{D}_{98 \%}=31.1 \mathrm{vs.} 30.6\right.$ Gy, $p<0.0001)$, less MU (2335 vs. 3454, $p<0.0001)$, and better HI ( 0.09 vs. $0.11, p<0.0001)$ and DSC $(0.85$ vs. $0.75, p<0.0001)$ values compared to IMRT. VMAT also resulted in better dosimetric parameters for the $\mathrm{BW} \mathrm{V}_{50 \%}(26.1 \% v s .33 .3 \%, p=0.0001)$ and the penile bulb $\mathrm{D}_{\text {mean }}(30.7$ vs. $31.1 \mathrm{~Gy}, p=0.008$ ).

Conclusions: Up to a $32 \%$ rate of protocol deviations with potential impact on tumour control and/or toxicity occurred in the considered clinical trial. Treatment planning with IMRT was the main cause of protocol deviations. Prospective RTQA protocols should be encouraged for future SBRT trials to prevent, if possible, protocol deviations before start of treatment.

\section{Lung stereotactic body radiotherapy with electromagnetic transponders for real-time tumor tracking \\ Type: Physics}

\section{Presenting Author:}

Maud Jaccard

Authors:

M. Jaccard ${ }^{1}$, A. Champion ${ }^{1}$, A. Dubouloz ${ }^{1}$, J. Plojoux ${ }^{2}$, P. Soccal ${ }^{2}$, R. Miralbell ${ }^{1}$, G. Dipasquale ${ }^{1}$, F. Caparrotti ${ }^{1}$

\section{Institutions:}

Geneva University Hospital

Department:

${ }^{1}$ Department of Radiation Oncology, ${ }^{2}$ Department of Pneumology

Aims: We report on the lung stereotactic radiotherapy treatments (SBRT) of 6 patients using the Calypso system for real-time tumour tracking.

Methods: Six patients were implanted with lung-specific electromagnetic transponders (EMT) near the tumour during diagnostic bronchoscopy using the SuperDimension Navigation System. Simulation for SBRT was performed in free breathing (FB), and also in deep inspiration breath hold (DIBH) for patients with suitable breathing capabilities. $60 \mathrm{~Gy}$ in 3,5 or 8 fractions were prescribed to the PTV, and delivered with two $6 \mathrm{MV}$ flattening filter free beams. Three patients were treated in DIBH, which allowed a better dose sparing of the lung and adjacent organs at risk compared to FB planning. Patients' setup in DIBH (resp. FB) at linac was performed using EMT position (resp. mean position) and CBCT imaging. We analysed patients positioning and fractions delivery using DIBH or FB and real-time tracking with EMT.

Results: The implant procedures were performed without side effects and EMT position in lungs remained stable over the entire course of treatment. Median setup time, including imaging at linac, was $10 \mathrm{~min}$ (range: 5-34 $\mathrm{min}$ ) and median session time was 13 min (range: 7-39 min). During treatments, EMT centroid positions were monitored real-time $(24 \mathrm{~Hz})$ by Calypso. SBRT was gated when EMT position exceeded predefined tolerance thresholds ( $3 \mathrm{~mm}$ in DIBH and up to $8 \mathrm{~mm}$ in FB), ensuring thereby the correct delivery of the dose to the tumour. Beams holds due to patient coughing or release of DIBH occurred with a median of 4 times per fractions.
Conclusion: Six patients were successfully implanted and treated with lung SBRT using tumour motion tracking via implanted EMT. Calypso showed full reliability to deliver SBRT to small moving target with confidence.

\section{Automated multi-metastases planning: the prescription isodose level re-visited}

Type: Physics

Presenting Author:

Nicoletta Lomax

Authors:

Lomax N, Alonso S, Leick M, Rogers S, Lutters G

Institutions:

Kantonsspital Aarau, Kantonsspital Baden

Department:

Radio-Onkologie Zentrum

Aims: In linac-based radiosurgery for multiple brain metastases, automatic single isocentre planning techniques are desirable for improved efficiency. The dosimetric advantages of different automated planning options within Elements 2.0 Multiple Metastases software, (SRS prescription optimization versus Normal Tissue Sparing (NTS) optimization) are evaluated and also compared to Elements 1.5. Further, we assess the optimal maximum dose relation (MDR, ratio of prescription

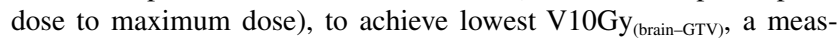
ure of normal brain sparing, (constraint V10Gy (brain-GTV) $_{\text {( }} \leq 10 \mathrm{~cm}^{3}$ per lesion).

Methods: 20 cases (total of 85 lesions) were planned using single isocentre dynamic conformal arcs with a single fraction, D99\%=20 Gy. For each case, 9 automatic plans were created with different optimizations: 4 plans with NTS settings $1-7$ and 5 plans with SRS prescription isodose level (IDL) settings from $65-85 \%$. Plan quality metrics (con-

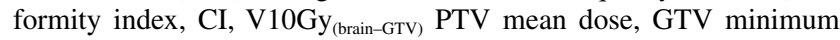
dose) are analysed.

Results: Increasing NTS, or selecting a lower IDL in SRS prescription mode both have the effect of increasing the PTV mean dose, whilst reducing the normal tissue volume receiving a significant dose,

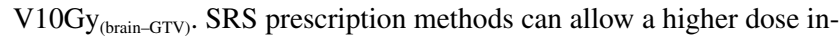
homogeneity within the target volume (lower MDR), however, setting IDL values below $70 \%$, or using the highest NTS setting, fails to further reduce the $\mathrm{V} 10 \mathrm{~Gy}_{\text {(brain-GTV). }}$. CI varied insignificantly with different optimizations and PTV mean dose was typically representative of GTV minimum dose. V10Gy is consistently reduced for Elements 2.0 compared with Elements 1.5 by a mean of $20 \%$.

Conclusion: V10Gy $y_{\text {(brain-GTV) }}$ can be reduced by appropriate selection of the MDR, accomplished by both NTS and SRS optimization methods, which may allow the safe treatment of larger tumors using a single fraction. The best normal tissue sparing was achieved for plans with a MDR of $70 \%$.

Evaluation and comparison of two commercial autosegmentation solutions for OARs in the male pelvis. Type: Physics

Presenting Author:

Sofia Celi

Authors:

S. Celi, S. Gomez, L. Braun, O. Riesterer, G. Lutters

Institutions:

Kantonsspital Aar Kantonsspital Aarau, Kantonsspital Baden

Department:

Radio-Onkologie Zentrum

Automation is one of the main foci in the modernization of the planning process. Two programs used in clinical routine are at our disposal 
for auto-segmentation: Velocity v.4.0 (Varian, atlas/model based approaches) and Eclipse v.15.5 (Varian, combined atlas/model based approach). Their performance is being evaluated for future clinical use. This first analysis concerns the segmentation of organs at risk of the male pelvis for the irradiation of the prostate.

The evaluation was conducted retrospectively on ten cases randomly picked from an RTOG study patient cohort. These patients underwent radiotherapy of the prostate and seminal vesicles and were contoured manually according to RTOG guidelines. Five representative cases were selected to complete the prostate atlas set of Eclipse and create a prostate atlas set in Velocity. Both atlas sets as well as the model-based segmentation by Velocity were tested on the patient cohort. The five organs-at-risk (OARs-bladder, femoral heads, penile bulb, and rectum) were then graded from 1 (unacceptable) to 5 (excellent) by two MDs. The auto-segmentation and the correction of the auto-segmented contours were timed. This time was compared to the average time necessary for contouring the same five OARs manually.

The Smart Segmentation module of Eclipse achieved a higher score rating for the five OARs of this treatment site. It was also assessed to be more flexible and transparent in clinical use. In average the resulting contours were graded partially acceptable to acceptable. Though generating auto-contours takes little time, correcting the contours takes longer than contouring the OARs of the male pelvis manually.

The disappointing times and partially acceptable contours are in line with the performance of auto-contouring modules in the male pelvis in other centers. However, an Eclipse ATLAS set including OARs conforming to the most crucial criteria to reduce uncertainties is still being worked on. Evaluations of both modules for other treatment sites, where different manual and automatic segmentation times are expected are also being conducted.

\section{Impact of skin: to-target distance on treatment plan quality for mixed beam radiotherapy}

Type: Physics

\section{Presenting Author:}

Torelli Nathan

Authors:

N. Torelli ${ }^{1}$, S. Mueller ${ }^{1}$, M. K. Fix ${ }^{1}$, M.F. M. Stampanoni ${ }^{2}$, P. Manser ${ }^{1}$

\section{Institutions:}

${ }^{1}$ Inselspital, Bern University Hospital, and University of Bern, ${ }^{2} \mathrm{ETH}$

Zürich and PSI, Villigen

Departments:

${ }^{1}$ Division of Medical Radiation Physics and Department of Radiation

Oncology, ${ }^{2}$ Institute for Biomedical Engineering

Aims: Mixed beam radiotherapy (MBRT) can outperform photon-only IMRT for targets which have a superficial part with a skin-to-target distance (STTD) smaller than $1 \mathrm{~cm}$. The aim of this work is to investigate for which range of STTD MBRT provides potential dosimetric benefits and to quantify whether or not the dose delivered to the skin is increased for MBRT compared to IMRT.

Methods: An academic situation consisting of a cubic water phantom which includes contours of several PTVs varying in the STTD from 0.5 up to $7.5 \mathrm{~cm}$ and two organs at risk (OARs) was defined. For each PTV, IMRT and MBRT plans were created without special attention of reducing the dose to the skin. An additional MBRT plan was created for each PTV aiming in achieving a similar dose to the most superficial $5 \mathrm{~mm}$ of the skin-by means of dose constraint in the optimization-as achieved with the corresponding IMRT plan. To estimate the skin dose, gafchromic film measurements were performed in a $140 \mu \mathrm{m}$-deep layer for the IMRT and MBRT plans with an STTD of $0.5 \mathrm{~cm}$.

Results: MBRT plans outperform IMRT plans in terms of PTV dose coverage, OARs sparing and volume of low dose bath for STTDs up to $3.5 \mathrm{~cm}$. However, the maximal dose delivered to a $0.5 \mathrm{~cm}$-thick skin is on average $30 \%$ higher for MBRT if no special constraint is given in the optimization. By setting an appropriate skin sparing constraint, the advantages of MBRT over IMRT diminish, but for STTDs up to $1.5 \mathrm{~cm}$ MBRT still gives dosimetric benefits compared to IMRT. Measurements show that the dose delivered to the $140 \mu \mathrm{m}$-deep layer is $300 \%$ higher for MBRT than for IMRT.

Conclusions: The results indicate dosimetric advantages for MBRT over IMRT for targets with a short STTD. The range of STTDs for which this is the case strongly depends on the maximal tolerated dose to specific skin layers. This work was supported by Varian Medical Systems.

\section{InSightive-a business intelligence solution for a paperless clinic \\ Type: Physics}

Presenting Author:

Samuel Peters

Authors:

S. Peters, M. Bovet, L. Plasswilm

\section{Institutions:}

Kantonsspital St. Gallen

Department:

Klinik für Radio-Onkologie

Aim: An essential part of managing a paperless clinic is how to maintain an overview of all tasks and processes without paper files or running sheets; and at the same time ensure that all necessary data is available in the required quality.

Within ARIA: (v.15.5) the tool InSightive (v.1.6) offers a platform that will allow such questions to be answered dynamically and in real time via dashboards by accessing all data stored in the ARIA database. Methods: In order to maintain an overview of the paperless processes the use of InSightive was limited for this project to the following questions:

How efficient are the electronic workflows?

What does data quality look like?

What does the patient volume look like?

In a first phase:, the available and ready-to-use dashboards were tested with the aim of finding the right dashboards for the questions mentioned. In a second step, these dashboards were analysed whether the displayed data are correct. Thirdly, these dashboards were adapted or new ones created from scratch.

Results: It quickly became apparent that the existing dashboards were unable to answer our questions-at least without making extensive adjustments. The analysis of the original and adapted dashboards showed that the data was often not precise and sometimes even wrong. This is because certain processes in ARIA are not exactly defined (e.g. when is the start of a task?) or because the dashboards are incorrectly programmed and therefore output incorrect data. This led to us having to limit ourselves to a very few dashboards which we had reprogrammed from scratch. The effort and training required to do this demands a deepened database and IT knowledge, as well as a lot of time.

Conclusion: InSightive is an analysis tool that offers the possibility to overview clinical data and processes easily and quickly. The tool itself and the provided dashboards fulfil this requirement in a very simple basis. However, if specific and detailed queries are needed, this is only possible with enormous effort and precise testing of the outcome. 
Visualizing the progression patterns of lymphatic metastasis in head and neck squamous cell carcinoma (HNSCC) to improve clinical target volume (CTV) definition

Type: Physics

\section{Presenting Author:}

Bertrand Pouymayou

Authors:

Pouymayou B, Koechli C, Balermpas P, Guckenberger M, Unkelbach J

\section{Institutions:}

University Hospital Zurich

Department:

Radiation Oncology

Aim: The CTV in HNSCC patients contains lymph node levels (LNL) that are at risk of harboring microscopic tumor even if they are not radiologically confirmed. The risk of microscopic involvement of LNL depends on the primary tumor (PT) characteristics, but also on the patient's individual location of macroscopic lymph node (LN) metastases. This latter aspect is insufficiently investigated in the literature.

Methods: We retrospectively analyzed $132 \mathrm{HNSCC}$ patients treated with definitive radio- or radiochemotherapy at our institution. For each patient, we recorded the ipsi- and contralateral involved LNLs (I to VII) on the planning CT and when available on FDG-PET, FNA cytology and MRI reports. This study includes PT (stage T1-T4) of the oropharynx (84), hypopharynx (28), oral cavity (10) and larynx (10). HPV status was reported when available. A graphical visualization linked to a SQL database was developed to display the frequency of involved LNLs combinations depending on risk factors. In addition, Bayesian networks (BN) were applied to model lymphatic progression and infer the probability of microscopic involvement of LNL, incorporating sensitivity and specificity of imaging.

Results: Among 76 lateralized oropharyngeal patients, levels II, III and IV were diagnosed positive in 67,25 , and 2 patients ipsilaterally, and 36,8 , and 0 patients contralaterally. However, in only 2 patients, the ipsilateral level III was involved while level II was not. Microscopic tumor progression follows the same patterns as the location of macroscopic metastases. While the frequency of ipsilateral level III involvement is $33 \%$, the BN analysis limits the risk of occult metastasis in level III to below $10 \%$ when level II is not involved.

Conclusions: The analysis of lymphatic progression patterns in $\mathrm{HN}$ SCC may allow for personalized and automated elective CTV definition. The risk of microscopic involvement of downstream levels is very low in patients without macroscopic metastases in upstream levels, possibly allowing CTV reduction for selected patients.

\section{CT radiomics differentiates levels of radiosensitivity in tumor subvolumes in head and neck cancer \\ Type: Physics}

\section{Presenting Author:}

Marta Bogowicz

Authors:

Bogowicz $\mathrm{M}^{1}$, Pavic $\mathrm{M}^{1}$, Riesterer $\mathrm{O}^{1,2}$, Finazzi $\mathrm{T}^{1}$, Garcia Schüler $\mathrm{H}^{1}$, Holz-Sapra $E^{1}$, Rudofsky L ${ }^{1}$, Glatz $\mathrm{S}^{1}$, Basler $\mathrm{L}^{1}$, Spaniol $\mathrm{M}^{1}$,

Hüllner $\mathbf{M}^{3}$, Guckenberger $\mathrm{M}^{1}$, Tanadini-Lang $\mathrm{S}^{1}$

\section{Institutions:}

${ }^{1,2}$ University Hospital Zurich, ${ }^{3}$ Kantonsspital Aarau

Department:

${ }^{1}$ Department of Radiation Oncology, ${ }^{2}$ Department of Nuclear Medicine, ${ }^{3}$ Center for Radiation Oncology

Aims: Radiomics was proposed as prognostic biomarker in head and neck cancer (HNC); however, most radiomics studies did not analyze potential intra-tumor heterogeneity. Here we investigated if radiomics can distinguish between tumor subvolumes with different levels of radiosensitivity.
Methods: We have retrospectively collected data from $31 \mathrm{HNC}$ patients treated with definitive radiochemotherapy, with the inclusion criteria: contrast-enhanced CT imaging prior to treatment, local tumor recurrence confirmed by biopsy and FDG PET imaging at the time of recurrence. Recurrence (g_rec) was contoured based on the PET image and the contours were transferred to the initial planning CT. Two regions were analyzed with radiomics: recurrence (overlap between primary tumor PT and g_rec) and control (PT minus g_rec). 162 intensity and texture features were extracted from planning CT. Principal component analysis and multivariate logistic regression with backward selection were used to distinguish between control and recurrence regions. The final model was tested in the cohort of 14 patients from phase II study selected based on the same inclusion criteria.

Results: The final model for subvolumes differentiation comprised two features, indicating that recurrence regions are more heterogeneous than controlled regions prior to any treatment. The model showed good performance in the validation cohort AUC $=0.88$ (95\%CI: $0.72-$ 1.00 ), sensitivity $=0.75$, specificity $=0.83$.

Conclusion: We have shown for the first time that pretreatment CT radiomics can differentiate levels of radiosensitivity in HNC. It is a first step towards radiomics-based dose painting.

\section{D dose reconstruction for prostate SBRT}

Type: Physics

\section{Presenting Author:}

Stefanie Ehrbar

Authors:

Ehrbar $\mathrm{S}^{1}$, Hagen $\mathrm{T}^{1}$, Lang $\mathrm{S}^{1}$, Poulsen $\mathrm{P}^{2}$, Guckenberger $\mathrm{M}^{1}$

\section{Institutions:}

${ }^{1}$ University Hospital Zurich, ${ }^{2}$ Aarhus University Hospita

Department:

${ }^{1}$ Department of Radiation Oncology, ${ }^{2}$ Department of Oncology and Danish Center for Particle Therapy

Purpose: To reconstruct the delivered dose distribution of prostate SBRT treatments accounting for patient-individual intra-fractional organ motion.

Methods: Five prostate cancer patients were treated with SBRT of $5 \times 7$ Gy to the prostate and with integrated boost of $5 \times 8$ Gy to the index lesion for 4 patients. Margins of 5 and $3 \mathrm{~mm}$ were used for the prostate and index lesion, respectively. During all fractions, the prostate motion was tracked with implanted radiofrequency beacons (Calypso, Varian). Gating with a 3-mm action threshold was applied with manual repositioning if necessary. The motion trajectories were used to create 4D dose reconstructions for each fraction: the treatment plan was split in partial arcs, which were recalculated with isocenter shifts according to the actual target offset, before they were summed up again. This workflow allowed to estimate the actually delivered 4D dose of each patient with gating and additionally the 4D dose if no gating would have been applied. Furthermore, all plans were re-optimized and simulated with zero safety margins.

Results: Reconstructed 4D doses without gating showed that patient movement overall would lead to a spread in dose delivery, with deviations ranging $+-16 \%$ in bladder Dmean and $+-12 \%$ in rectum Dmax. Gating eliminated outliers and reduced this spread to $+-5 \%$ and $+-6 \%$, respectively. For the prostate dose coverage (D95), reductions of 0-9\% without gating were observed, but no reduction $(0-1 \%)$ with gating. Removing the safety margin would on average reduce the bladder dose by $39 \%$, but make the prostate D95 more susceptible (no gating: $1-13 \%$, gating: $0-2 \%$ ).

Conclusion: The effect of intra-fractional prostate motion was modelled with 4D dose reconstructions. All patients had a dosimetric benefit from gating, which allowed for an accurate dose delivery during prostate SBRT. Motion mitigation is especially important for hypofractionated treatments and small safety margins. 
CT based lymph nodes radiomics to predict 12-months eventfree survival in stage IIIA NSCLC; SAKK 16/00

Type: Physics

Presenting Author:

Diem Vuong

Authors:

D. Vuong ${ }^{1}$, M. Bogowicz ${ }^{1}$, S. Denzler ${ }^{1}$, C. Oliveira ${ }^{1,2}$, R. Foerster $^{1}$,

F. Amstutz ${ }^{1}$, H. S. Gabryś1, J. Unkelbach ${ }^{1}$, S. Hillinger ${ }^{3}$,

S. Thierstein ${ }^{4}$, L. Basler ${ }^{1}$, E. Eboulet ${ }^{4}$, S. Peters ${ }^{5}$, M. Pless 6 ,

M. Guckenberger ${ }^{1}$, S. Tanadini-Lang ${ }^{1}$

\section{Institutions:}

${ }^{1,3}$ University Hospital Zurich, ${ }^{2}$ Queen's University, Kingston, Ontario, ${ }^{4}$ Swiss Group for Clinical Cancer Research (SAKK) Coordinating Center, Bern, ${ }^{5}$ Centre Hospitalier Universitaire Vaudois, Lausanne,

${ }^{6}$ Kantonsspital Winterthur

Department:

${ }^{1}$ Department of Radiation Oncology, ${ }^{2}$ Department of Oncology, Cancer Center of Southeastern Ontario, ${ }^{3}$ Department of Thoracic Surgery, ${ }^{5}$ Department of Oncology, ${ }^{6}$ Department of Medical Oncology

Aims: Radiomics is a growing research field but is often limited to single institution imaging data. The sensitivity of radiomics features to e.g. scanning settings present in multi-centric imaging data is disadvanteous for robust radiomic models. We built a robust CT lymph nodes radiomics model to predict 12-months event-free survival (EFS) trained on a multi-centric imaging dataset and validated it on an external homogeneous dataset.

Methods: 74 NSCLC patients were selected from a Swiss multi-centric prospective randomized phase 3 trial (IIIA/N2, SAKK-16/00, neoadj. radio/chemoradiotherapy prior surgery, 14 sites). Patients received CT scans with similar imaging protocol (non-contrast, FBP,standard kernel). ROI was a combined lymph nodes structure. 1404 radiomic features were fed into a principal component analysis. Surrogate features representing $95 \%$ data variance were included in a 12-months logistic regression using LASSO. 5-fold cross validation was used to study the performance. The final model was validated on a separate internal dataset ( $n=32$, IIIA/N2/IIIB). Performance measure was area under the roc curve (AUC).

Results: The final model ( 3 features) had a performance of $\mathrm{AUC}=0.722(95 \% \mathrm{CI}=0.60-0.84)$ and $0.78(0.59-0.97)$ for training and validation, resp.

Conclusion: A 12-months EFS CT radiomics model from combined lymph nodes structure was built for NSCLC patients. This model could be validated on homogeneous single-institution data with good performance.

\section{Efficiency and safety improvements for proton treatment plan QA by automation \\ Type: Physics}

\section{Presenting Author:}

Till Böhlen

Authors:

Böhlen $\mathrm{TT}^{1}$, Rosas $\mathrm{S}^{1}$, Lowe $\mathrm{M}^{2}$, Bizzocchi $\mathrm{N}^{1}$, Lomax $\mathrm{AJ}^{1}$,

Weber DC ${ }^{1}$, Hrbacek J

\section{Institutions:}

${ }^{1}$ Paul Scherrer Institut, Villigen, ${ }^{2}$ University of Manchester

Department:

${ }^{1}$ Centre for Protontherapy

Background: Meticulous treatment planning QA is essential to deliver safe and high-quality proton treatments. Repetitive and extensive plan QA binds significant amounts of resources in clinical routine and is prone to human error. This work presents and evaluates tools developed at our clinic to extend, automatize and standardize the physicists' and clinicians' plan review.

Method \& materials: Using the scripting capabilities of the commercial TPS Eclipse ${ }^{\mathrm{TM}}$ (Varian), two plugins for plan QA were developed. A Plan Checker plugin verifies parameters and properties of plans. The comprehensive checks cover the planning CT, the structure set, and the plan itself and generate more than 150 different colour-coded messages which are classified according to their severity (INFO, PASSED, TO CHECK, WARNING, ERROR). Checks encompass amongst others: correct labelling (e.g. switching of left and right of organs), plan approval by authorized personnel, correct patient orientation, correct field tolerance tables, reasonable air gaps, HU overrides, plan and beam model parameters, the degree of field modulation, normalization, plan and field maxima and their positions, etc. A second tool evaluates clinical objectives for a given plan or plan sum and indicates by colour-coding if they are met. It allows for easy template generation and can obtain data from the clinicians' prescription. Plan uncertainty can be additionally evaluated.

Results: Both tools extend and standardize the clinically performed plan checks while leading to a considerable time-reduction when performing the physicists' and clinicians' plan review. At the same time they lead to a reduction of repetitive, human error-prone work in clinical routine.

Conclusions: TPS plugins which automatize parts of the plan QA allow to create flexible plan checks and can be tailored to institutional needs and practices. They can contribute to increase safety, standardization and efficiency for proton therapy treatment planning.

\section{Development of an in-house VMAT treatment planning} Type: Physics

\section{Presenting Author:}

Alisha Dütschler

Authors:

A. Duetschler ${ }^{1}$, S. Mueller ${ }^{1}$, P. Manser ${ }^{1}$, D. M. Aebersold ${ }^{1}$, M.F.M. Stampanoni ${ }^{2}$, M. K. Fix ${ }^{1}$

\section{Institutions:}

${ }^{1}$ Division of Medical Radiation Physics and Department of Radiation Oncology, Inselspital, Bern University Hospital, and University of Bern, ${ }^{2}$ Institute for Biomedical Engineering, ETH Zürich and PSI, Villigen

Department:

Division of Medical Radiation Physics

Aims: Volumetric modulated arc therapy (VMAT) is the current stateof-the-art treatment technique for radiotherapy. Commercial optimizers are used to generate clinical VMAT plans, however, their implementation provides a limited flexibility for the user. In order to overcome this limitation, a new treatment planning process (TPP) was developed. Methods: The in-house developed TPP is based on Monte Carlo calculated beamlet dose distributions. A hybrid column generation and simulated annealing based direct aperture algorithm (HDAO) directly optimizes the MLC leaf positions and monitor units of discrete control points describing the arcs for VMAT. Deliverable VMAT plans for an academic situation and a clinically motivated bladder, head and neck and prostate case were generated with the new TPP and compared to plans optimized with the commercial Eclipse photon optimizer (version 13.6).

Results: The HDAO plan for the academic situation showed a decrease in mean dose by $19.1 \%$ for the single OAR and a decrease in the PTV homogeneity of $3.0 \%$ when compared to the Eclipse plan. The PTV coverage, the maximal dose to serial OARs and the dose to normal tissue are similar for the HDAO and Eclipse plans for the clinically motivated cases. However, the mean dose in parallel OARs is on average $8.6 \%$ smaller for HDAO plans compared to Eclipse plans. The delivery time for HDAO and Eclipse optimized plans is equal. 
Conclusion: A TPP was successfully developed for deliverable VMAT plans. Comparisons with Eclipse optimized plans suggest similar plan quality with some benefits for parallel OARs for the investigated cases. The flexible and new TPP allows the implementation of further functionalities in future projects.

This work was supported by Varian Medical Systems.

\section{End to end dosimetric and geometric accuracy of a magnetic resonance guided linear accelerator \\ Type: Physics}

\section{Presenting Author:}

Luisa Sabrina Stark

Authors:

L. S. Stark, M. Baumgartel, S. Ehrbar, J. Krayenbühl, M. Zamburlini, M. Chamberlain, N. Andratschke, M. Guckenberger, L. Wilke and S. Tanadini-Lang

Institutions:

University Hospital Zurich

Department:

Radio oncology

Purpose: Magnetic resonance guided linear accelerators (MR-Linac) allow for online imaging during the treatment and enable gating on the tumor position. To assess the overall dosimetric and geometric accuracy of the MRIdian MR-Linac (Viewray), end-to-end tests with three different phantoms were performed for static and moving targets.

Methods and materials: Six different treatment plans were measured in the static inhomogeneous thorax phantom (CIRS) using a Farmer chamber. Radio chromic film measurements were performed in the Lucy 3D QA phantom (Standard Imaging) for an IMRT plan with 11 fields. The gating performance of the MR-Linac was evaluated in the longitudinal direction in the dynamic thorax phantom (CIRS) using a 10 -field IMRT plan. Stripes of radio chromic films were inserted into a target rod moving longitudinally with an amplitude of $15 \mathrm{~mm}$ within a body-like support structure. The gross tumor volume (GTV) was enlarged by $3 \mathrm{~mm}$ to define the gating window.

Results: Mean dose deviation in the CIRS phantom was $0.7 \% \pm 0.4 \%$ compared to the planning system. The accuracy was equally good in water, lung and bone tissue surrogate. For static treatments the gamma evaluation of films in the Lucy phantom was repeated showing passing rates of $97 \%$ and $98.5 \%$ for a $5 \% / 1 \mathrm{~mm}$ criteria and a passing rate of $100 \%$ for a $5 \% / 1.5 \mathrm{~mm}$ criteria. The gated dose distribution showed a longitudinal offset of $1 \mathrm{~mm}$ relative to the static treatment and an offset of $1.5 \mathrm{~mm}$ compared to the planned dose distribution. The measured dose maximum was $2 \%$ lower in the gated treatment compared to the static treatment and $4 \%$ lower than the planned dose.

Conclusion: The MRIdian showed a high geometric and dosimetric accuracy which allows for precise stereotactic body treatments and accurate gating for moving targets.

\section{Modeling and measuring clinical electron beams in magnetic fields: investigating the potential of future MR-guided electron therapy}

Type: Physics

\section{Presenting Author:}

Reto Kueng

Authors:

R. Kueng ${ }^{1}$, B. M. Oborn ${ }^{2,3}$, N. Roberts ${ }^{2}$, T. Causer ${ }^{2}$, S. Mueller ${ }^{1}$, M.F.M. Stampanoni ${ }^{4}$, P. Manser ${ }^{1}$, P. J. Keall ${ }^{4}$, M. K. Fix ${ }^{1}$

\section{Institutions:}

${ }^{1}$ Inselspital, Bern University Hospital, and University of Bern,

${ }^{2,3}$ University of Wollongong, Wollongong NSW, ${ }^{4}$ University of Zurich and Swiss Federal Institute of Technology (ETH), Zurich, ${ }^{5}$ Sydney Medical School, University of Sydney
Department:

${ }^{1}$ Division of Medical Radiation Physics and Department of Radiation Oncology, ${ }^{2}$ Centre for Medical Radiation Physics, ${ }^{3}$ Illawarra Cancer Care Centre, ${ }^{4}$ Institute for Biomedical Engineering, ${ }^{5}$ ACRF Image $\mathrm{X}$ Institute

Aims: Future integrated MR guided radiotherapy systems might profit from the availability of an electron beam mode. The aim is to model and experimentally confirm the dosimetric impact of magnetic fields on clinical electron beams for different beam energies, magnetic field strengths including two relative orientations in a water equivalent phantom.

Methods: A multiple source Monte Carlo (MC) beam model was commissioned for a Varian Clinac $2100 \mathrm{C}$ linear accelerator and applied to simulate the particle transport including a magnetic field with the Geant4 MC toolkit. A permanent magnet device was used to generate a strong magnetic field up to $0.7 \mathrm{~T}$ encompassing a solid water phantom. Gafchromic EBT3 film was placed in the phantom for dose measurements of 6,12 and $20 \mathrm{MeV}$ electron beams in a perpendicular and parallel magnetic field orientation and a zero magnetic field reference setup.

Results: Film data confirmed MC predictions of substantial deflection of the electron beam in a perpendicular magnetic field due to the Lorentz force for all three initial energies compared to reference measurements. For a parallel magnetic field, a dose enhancement up to $100 \%$ (6 MeV beam, 0 vs. $0.7 \mathrm{~T}$ magnetic field) was observed in the dose profiles at different depths in the phantom.

Conclusions: An experimental and corresponding in-silico framework to measure and simulate clinical electron beams in magnetic fields of different strengths and relative orientations was established and successfully tested.

\section{Implications of respiratory motion variability for the ITV approach in PBS proton therapy}

Type: Physics

\section{Presenting Author:}

Miriam Krieger

Authors:

Miriam Krieger ${ }^{1,2}$, Alina Giger ${ }^{3}$, Damien C Weber ${ }^{1,4,5}$, Tony Lomax ${ }^{1,2}$, Ye Zhang ${ }^{1}$

\section{Institutions:}

${ }^{1}$ Paul Scherrer Institute, ${ }^{2}$ ETH Zürich, ${ }^{3}$ University of Basel, ${ }^{4}$ University Hospital Zürich, ${ }^{5}$ Inselspital Bern

Department:

${ }^{1}$ Centre for Proton Therapy, ${ }^{2}$ Department of Physics, ${ }^{3}$ Center for medical Image Analysis \& Navigation, ${ }^{4}$ Department of Radiation Oncology, ${ }^{5}$ Department of Radiation Oncology

Introduction: Motion management is a key component for pencil beam scanned (PBS) proton therapy. Currently, treatment planning and target definition for mobile tumours are still based on a single 4DCT without considering variable breathing. We aim in this analysis to assess the dosimetric impacts of motion variability, and propose a probabilistic ITV definition to account for respiratory variabilities.

Methods: CTs from two lung patients were warped using deformation vectors extracted from 4DMRI datasets of two volunteers, resulting in four pseudo-4DCT datasets covering 40 breathing cycles each. A percentage map was defined by overlaying the first 20 single-cycle ITVs. Threshold percentages $(\mathrm{x}=0 \%, 25 \%, 50 \%$, or $75 \%)$ were selected to define probabilistic ITVs (ITVx).

Two-field PBS proton treatment plans were optimised on the four ITVs, and 4D dose distributions were calculated using the second 20 breathing cycles. In order to minimise the influence of interplay effect, $9 \times$ volumetric rescanning was applied. 
Results: The volumes of the single-cycle ITVs vary up to $20 \%$ around their mean. Averaged over all four datasets, the median CTV V95\% for the ITV00, ITV25, ITV50, and ITV75 are 98.7\%, 97.6\%, 97.2\%, and $94.3 \%$ respectively. In the same order, median D5-D95\% are $9.4 \%$, $10.1 \%, 10.6 \%$, and $12.0 \%$. The median ipsilateral mean lung doses are $14.8 \%, 13.8 \%, 13.2 \%$, and $12.4 \%$.

Conclusion: Breathing variations can have a significant impact on the ITV definition. Our probabilistic ITV definition can effectively account for variable motion, resulting in acceptable target coverage and substantial dose reduction to the involved lung. The ITV50 approach provided the best compromise between the two clinical objectives.

\section{Quality assurance for the adaptive workflow on the MRI linac Type: Physics}

\section{Presenting Author:}

Lotte Wilke

Authors:

L. Wilke, S. Ehrbar, M. Bogowicz, J. Krayenbühl, N. Andratschke, M. Guckenberger, S. Tanadini-Lang

\section{Institutions:}

Universitätsspital Zürich

Department:

Klinik für Radio-Onkologie

Aims: The new MRidian MR Linac enables online adaptive treatment for daily changing anatomy of the patient. For these modulated treatments, no measurement of the treatment plan can be performed prior to treatment. Besides performing a secondary calculation within the system, we set up a chain of additional tests to ensure a safe treatment. Methods: For all patients the original treatment plan as well as all adapted plans were measured on an IMRT verification phantom (Delta 4 , Scandidos). During the adaptive process, we did a point dose verification in an independent Software (Radcalc). Additionally, we calculated a value that is representative of the integral dose delivered to the patient and compared this value to the original plan. To ensure that the electron densities are correctly assigned to the new MRI, we compared the equivalent pathlength of the original and the adapted plan.

Results: For the first 10 Patients, all measurements of the original plan passed the gamma-analysis with a $3 \% / 3 \mathrm{~mm}$ criterion of on average 99.9\% (range $99.6-100 \%$ ). The adapted plans had equally good passing rates (average $99.8 \%$, range $97.2-100 \%$ ). For $89 \%$ of the plans the independent point dose calculation agreed within $10 \%$ with the original plan one (average $5.9 \%$, range $0.3-21.4 \%$ ). Values above $10 \%$ were connected to a lung treatment, where the scatter is incorrectly taken into account in the point dose calculation. The value representing the integral dose also agreed within $10 \%$ between the original plan and the treated adapted plan (average $4.0 \%$, range $0.26-9.45 \%$ ). The effective pathlength differed less than $2 \mathrm{~cm}$ from the original plan except for one case, where the change was traced back to a change in anatomy.

Conclusion: We set up a QA chain for the online adaptive planning on the MRIdian MR Linac which ensures a save treatment of the patient.

\section{Influence of magnetic field transport parameters on accuracy and efficiency of electron transport in EGSnrc \\ Type: Physics}

\section{Presenting Author:}

Gian Guyer

Authors:

G. Guyer ${ }^{1}$, R. Kueng ${ }^{1}$, W. Volken ${ }^{1}$, M.F. M. Stampanoni ${ }^{2}$, P. Manser ${ }^{1}$, M. K. Fix ${ }^{1}$

Institutions:

${ }^{1}$ Inselspital, Bern University Hospital and University of Bern, ${ }^{2} \mathrm{ETH}$

Zürich and PSI, Villigen
Department:

${ }^{1}$ Division of Medical Radiation Physics and Department of Radiation Oncology, ${ }^{2}$ Institute for Biomedical Engineering

Aims: In context of MR guided radiotherapy, accurate and efficient dose calculation and therefore describing the propagation of electrons in a magnetic field is essential. The aim is to quantify the influence of the EGSnrc magnetic field transport parameters on the electron transport in a magnetic field in a local spherical geometry and a macroscopic phantom. Methods: The EGSnrc 2019 version provides two different macros for handling magnetic fields: the EMF macro and a novel enhanced EEMF macro. Both are applied to calculate the deflection angle of 15 $\mathrm{MeV}$ electrons in a sphere ( $3 \mathrm{~mm}$ radius) due to a perpendicular 1.5 $\mathrm{T}$ magnetic field in vacuum and water with varying maximal electron step size. The deflection angles are compared to theoretical calculations. Next, the same parameters are used to calculate the dose in a water phantom with a $1 \mathrm{~cm}$ air slab in $1 \mathrm{~cm}$ depth for a $15 \mathrm{MeV}$ electron pencil beam with a magnetic field of $1.5 \mathrm{~T}$ perpendicular to the incident beam direction. The resulting dose distributions are investigated by comparing relative dose differences in the air slab.

Results: For the EMF, varying the step size shows substantial differences in the deflection angle with an error up to $19 \%$ compared to the theoretical calculation. The EEMF shows only differences of up to $0.2 \%$. The EMF shows dose differences of up to $6 \%$ in air but only $0.3 \%$ for a small step size. For the EEMF, the dose differences in air are up to $0.2 \%$ for all step sizes. The EMF computation time is $30 \%$ faster than the EEMF for the same step size but increases exponentially with decreasing step size.

Conclusion: The novel EEMF shows more accurate results for electron transport in a magnetic field when compared to EMF with the same step size. Although EMF can yield as accurate results as EEMF with a small step size, due to efficiency, EEMF should be preferred over EMF

\section{Dynamic trajectory radiotherapy versus HyperArc: treatment plan comparisons for tumors in the brain \\ Type: Physics}

\section{Presenting Author:}

Peter Manser

Authors:

P. Manser, S. Mueller, D. Frei, W. Volken, D. Terribilini,

D. Frauchiger, A. Joosten, D. Henzen, E. Herrmann, D. M. Aebersold, M. K. Fix

Institutions:

Inselspital, Bern University Hospital, and University of Bern Department:

Division of Medical Radiation Physics

Purpose: HyperArc applies a pre-defined set of non-coplanar arcs for treatments of brain tumors, while dynamic trajectory radiotherapy (DTRT) additionally includes dynamic couch and collimator rotations during beam on to increase degrees of freedom for the selection of beam directions. The aim of this work is to investigate the potential benefit of DTRT compared to HyperArc for brain tumors.

Materials \& methods: Five clinically motivated brain cases are included in this study. The target volumes range from 3 to $270 \mathrm{~cm}^{3}$ and are located at different areas in the brain with according organs at risk (OARs). A framework utilizing the Eclipse Scripting Research API was used to determine gantry-table and gantry-collimator paths based on contoured structures. These paths served as input for the multi-leaf sequence optimization using a research version of the VMAT optimization algorithm in Eclipse. Additionally, a HyperArc treatment plan was generated for each of the five cases. Resulting dose distributions for DTRT and HyperArc plans were compared based on DVH parameters. The deliverability of DTRT dose distributions was confirmed by gafchromic film measurements. 
Results: Comparing DVHs for the target shows that coverage is similar for all brain cases. On average over all five cases mean doses for parallel OARs and near maximum doses for serial OARs improved for DTRT treatment plans by $5.5 \%$ and $5.0 \%$ relative to the prescribed dose, respectively. Measured and calculated doses show a gamma passing rate $>99.5 \%$ for $2 \% / 2 \mathrm{~mm}$ criteria and a threshold of $10 \%$.

Conclusions: Depending on the case and on the OAR considered, the DTRT treatment plan generally performs better or similar compared to the HyperArc plan. The results demonstrate that DTRT has a great potential to reduce dose to OARs, while target coverage is preserved compared to HyperArc. This work was supported by Varian Medical Systems.

\section{A lean but still robust plan quality assurance program for stereotactic radiotherapy. Our experience with a redundant workflow \\ Type: Physics}

\section{Presenting Author:}

Enrico Barletta

Authors:

E. Barletta ${ }^{1,2}$, H. Haerle ${ }^{1}$, A. Clivio ${ }^{2}$, O. Sommer ${ }^{1}$, H. Nguyen ${ }^{2}$, C. Negreanu ${ }^{1}$

\section{Institutions:}

${ }^{1}$ Kantonsspital Winterthur, ${ }^{2}$ Rüti Zürich-Ost-Linth AG, Rüti ZH Department:

${ }^{1}$ Department of Radiation Oncology, ${ }^{2}$ Zentrum für Radiotherapie

Aims: Stereotactic treatments are an established treatment concept in Radiotherapy (RT). In recent years, the application of this technique has expanded substantially. This is mainly due to the development of the Image Guided RT (IGRT) techniques, which allow for a secure administration in organs not considered in the past for stereotactic RT, because of lack of positioning precision.

With the widening of the application field, the new arising challenge is to speed up the Plan Quality Assurance (QA) procedures without reducing safety. Film Dosimetry is still nowadays the gold standard for Plan QA in this field, but it is time-consuming and error prone.

The aim of this work is to compare different stereotactic RT QA methods with respect of precision and time optimization.

Methods: Ten stereotactic RT plans were retrospectively verified with five different methods, namely: Film Dosimetry (FD), Portal Dosimetry (EPID), dose verification by ionization chamber in a solid phantom, PTW Octavius 4D SRS (O4D) and Varian Mobius 3D offline recalculation. The results were analyzed in terms of precision and time requirements.

Results: Using FD as a benchmark, the comparison of the different QA techniques shows substantial agreement in the results, in particular for the O4D Phantom. The O4D results, strengthened by the other faster verification measurements, offer the same level of robustness in accepting or rejecting a stereotactic RT plan as with FD.

Conclusion: This study highlights the possibility of carrying out lean Plan QA verification procedures without a loss of accuracy. Performing redundant measurements increases the time of raw measurements, but allows to substantially shortening the total waiting time before accepting or rejecting a stereotactic RT plan in comparison with FD.

\section{Extended EPID commissioning for reliable plan verification of volumes well below 1cc}

Type: Physics

\section{Presenting Author:}

Anisoara Socoliuc Toquant

Authors:

A. Socoliuc Toquant, K. Buchauer, H. Schiefer, L. Plasswilm
Institutions:

Kantonsspital St. Gallen

Department:

Klinik für Radio-Onkologie

Aims: Among different methods for patient QA, one practical approach is the accelerator's EPID. Purpose of this work is to extend the $3 \mathrm{~cm}$ commissioned size to stereotactic volumes well below $1 \mathrm{cc}$ and demonstrate the clinical usefulness.

Methods: Portal Dosimetry commissioning data were acquired on $6 \mathrm{FFF}$ and 10FFF STX True Beams for static fields between $40 \times 40$ and $1 \times 1 \mathrm{~cm}$. This includes the generation of a relative output factor table $(\mathrm{OF})$ of the mean central pixel value determined during EPID irradiation. Extra- and intracranial stereotactic complex VMAT plans with target volumes between 0.15 and $13 \mathrm{cc}$ (collimation range $0.6-3 \mathrm{~cm}$ ) were verified before and after the $\mathrm{OF}$ adjustment. The output factors were adjusted to bring measurement and prediction to agreement. Correlations between parameters specific to dynamic plan delivery and dosimetric QA results were under investigation.

Results: For field sizes below $3 \times 3 \mathrm{~cm}$ large differences between calculated PDIP and measured EPID fluences are noticed. Surprisingly, $10 \mathrm{FFF}$ showed less pronounced dosimetric disagreements compared to $6 \mathrm{FFF}$, where for fields of $1 \times 1 \mathrm{~cm}$ the discrepancy was up to $36 \%$. With the modifications of output factors the fluence differences from prediction to measurement went well below $0.5 \%$. A net average increase in gamma passing rate, for 3\%-1 mm evaluation criteria, from 95.7 to $98 \%$ was obtained. As comparison, film-based plan verification lead to an average gamma-passing rate of $99.1 \%$ for a subset of SRS cases. Despite different modulation complexity scores, leaf and jaw opening statistics for the intracranial stereotactic plans, a clear correlation to the plan gamma-passing rate could not be established. In contrast, lung stereotactic plans showed correlation that is more pronounced.

Conclusion: Adjustment of the EPID prediction algorithm allows patient QA also for plans with effective field sizes below $1 \times 1 \mathrm{~cm}$. The verification results are on par with other verification methods used in our clinic.

\section{Results of a feed-back learning process for RapidPlan} knowledge based planning

Type: Physics

\section{Presenting Author:}

Antonella Fogliata

Authors:

Fogliata A. ${ }^{1}$, Cozzi L. ${ }^{1,2}$, Reggiori G. ${ }^{1}$, Stravato A. ${ }^{1}$, Lobefalo F. ${ }^{1}$, Franzese C. ${ }^{1}$, Scorsetti M.1,2

\section{Institutions:}

${ }^{1}$ Humanitas Research Hospital and Cancer Center, Milan, Italy,

${ }^{2}$ Humanitas University, Milan, Italy

Department:

${ }^{1}$ Radio-oncology

Aim: The knowledge based planning KBP capability to harmonize plan quality depends on the quality of the model, configured with selected plans and defined optimization objectives to estimate the DVH for organs at risk. This work will determine if the performance of a KBP model (RapidPlan, Varian, RP) could be improved with a feedback learning process, i.e. if plans generated by a RP model could be used as new input to re-train the model and achieve better performances.

Methods: Clinical VMAT plans from 83 patients presenting head and neck cancer were selected to train a RP model, RP-1. The same 83 patients were re-planned using the RP-1 model; the resulting plans were used as input to train a novel model, RP-2. The same optimization objectives were selected in the two models. The models quality was assessed through: the coefficient of determination $\mathrm{R}^{2}$, describing how 
the regression model represents the training data, and the mean squared error MSE between original and estimate. In addition, the regression plots for the various organs were analysed and compared. The two models were then validated on a cohort of 20 different patients, with a dosimetric comparison.

Results: The RP-2 model presented an improvement relative to RP-1, with higher $\mathrm{R}^{2}$, lower MSE values and better regression plots. A deeper analysis on the geometric features generated in the model allowed to better understand the model ability to capture the interpatient variability. From the validation process, plans generated with RP-2 presented, for parallel organs, lower mean doses of about $1 \%$ for RP-2 relative to RP-1 plans (with larynx showing a reduction of 5\%), significant. For the serial organs, on the contrary, an increase of the $D_{1 \%}$ of $3-4 \%$ was shown, although not significant.

Conclusion: A refined, with a feed-back process, RP model allowed to generate plans with improved quality, firstly in terms of model, but also in terms of plan quality.

Predicting survival and local control after radio-chemotherapy in locally advanced head and neck cancer by means of CT based radiomics

Type: Physics

Presenting Author:

Cozzi Luca

Authors:

Cozzi L. ${ }^{1,2}$, Fogliata A. ${ }^{1}$, Franzese C. ${ }^{1}$, Scorsetti M. ${ }^{1,2}$

\section{Institutions:}

${ }^{1}$ Humanitas Research Hospital and Cancer Center, Milan, Italy,

${ }^{2}$ Humanitas University, Milan, Italy

Department:

${ }^{1}$ Radio-oncology

Aim: This work aim to appraise the ability of a radiomics signature of CT based radiomics features to predict the clinical outcome after definitive radio-chemotherapy of stage III-IV head and neck cancer.

Methods: 110 patients presenting with advanced head and neck tumour were retrospectively selected. Radiomics texture features were extracted from the gross tumor volumes contoured on planning CT images. The cohort of patients was randomly divided into a training (70 patients) and a validation (40 patients) cohorts. Textural features were correlated to overall survival OS, progression free survival PFS, and local control LC data to build predictive models. All the significant predictors at univariate analysis were included in a multivariate regression model to stratify the cohort of patients in low- and high-risk sub-groups. The quality of the models was appraised by means of the concordance index $(\mathrm{CI})$. The models were tested against the validation cohort not used for the training.

Results: A signature with 3 features was identified as predictive of OS, with $\mathrm{CI}=0.88$ and 0.90 for the training and validation cohorts respectively. A signature with 2 features was identified for progression free survival, PFS, $(\mathrm{CI}=0.72$ and 0.80$)$. Two features characterized also the signature for local control, $\mathrm{LC},(\mathrm{CI}=0.72$ and 0.82$)$. In all cases, the stratification in high- and low-risk groups for the training and validation cohorts lead to significant differences in the actuarial curves. In the validation cohort the mean OS time resulted in $78.9 \pm 2.1$ vs $67.4 \pm 6.0$ months in the low- and high-risk groups respectively; the mean PFS time resulted in $73.1 \pm 3.7$ and $50.7 \pm 7.2$ months, while the mean LC time was $78.7 \pm 2.1$ and $63.9 \pm 6.5$ months.

Conclusion: CT based radiomic signatures were identified which correlate with survival and control after radio-chemotherapy and allowed to identify low- and high-risk groups of patients.
End-to-End testing method for SRS patient QA based on a new multidetector array and comparison with two other techniques Type: Physics

Presenting Author:

Konrad Buchauer

Authors:

K. Buchauer, A. Socoliuc Toquant, L. Plasswilm, H. Schiefer

Institutions:

Kantonsspital St. Gallen

Department:

Klinik für Radio-Onkologie

Aims: The accuracy and practicability of the new SRS MapCheck high resolution mutidetector array was tested and an intercomparison with our current methods for patient QA, namely film dosimetry and EPID fluence measurements was performed.

Methods: Multidetector array SRS MapCheck (SunNuclear), StereoPHAN (SunNuclear) based film dosimetry with the Film QA Pro software (Ashland) and EPID (Varian) were used. Acceptance criterion for patient QA is 3\%/1 mm with $95 \%$ gamma pass rate. Evaluation of all methods was performed for 10 SRS brain met cases $(0.49 \mathrm{cc}$ mean volume). In addition, SRS MapCheck verification method was extended to further 10 SBRT lung cases (22cc mean volume) and 5 mixed SBRT cases for larger volumes (60 $\mathrm{cc}$ mean volume) including adrenal gland, bile duct, prostate, pelvis. Regarding EPID, a tuning of the image prediction algorithm had to be performed first.

Results: For the 10 SRS brain met's cases the SRS MapCheck showed an average gamma pass rate of $97.0 \% \pm 2.3$. Two cases were outside of the clinical $95 \%$ pass rate criterion with $92.4 \%$ and $93.0 \%$. These cases are influenced by low-resolution fluence in the QA plans due to planning system memory restrictions. The 10 lung cases yielded an average pass rate of $98.4 \% \pm 0.6$ and the 5 other cases an average of $97.5 \% \pm 2.1$. Film-based dosimetry for brain cases revealed a pass rate of $99.1 \% \pm 0.8$, where as EPID measurements pre-optimization only $90.1 \% \pm 11.3$, and post-optimization $97.5 \% \pm 1.9$.

Conclusion: All three measurement methods performed the mixture of clinical cases accurately. In terms of workflow, EPID verification is very practical as it used the on-board detector. The SRS multidetector array and film dosimetry are well comparable in terms of required work amount, when defined procedures in film preparation and a template software evaluation are used. Based on accuracy, resolution and workload, film dosimetry remains, nevertheless, the gold standard for demanding cases in clinical use.

Benefits of an additional MRI for adaptive HDR-brachytherapy in gynecological cancers

Type: Physics

Presenting Author:

Klaus Luchs

Authors:

Luchs K, Tsikkinis A, Cihoric N, Terribilini D, Lössl K

Institutions:

Inselspital Bern, Bern University Hospital

Department:

Department of Radiation Oncology

Objective: To evaluate the benefit of using MRI imaging, after CTbased applicator positioning, for planning and contouring.

Materials and methods: Between Jan and May 2019, 17 patients (pts) with gynecological cancers were treated with MRI-assisted HDR-brachytherapy, following EBRT. 11 pts with cervical cancer were treated using standard ring applicators $+/$ - interstitial implants and 6 pts with vaginal or vulvar cancer were treated using freehand interstitial implants. Primarily all pts received a CT-scan for treatment 
planning. After the HDR-applicators were implanted, they received an MRI for matching purposes in the planning system. Contouring and treatment planning was performed by an experienced radio-oncologist (CTV_HR). Separately a resident physician, with radiology experience, contoured a CTV, based on the CT-scan (CTV_CT), and a second CTV, based on the post-implant MRI (CTV_MRI). All CTV volumes, dosimetric parameters (ICRU 89/ABS guidelines for interstitial vaginal BT) and doses to the organs at risk (OaR) (rectum, sigmoid, bowel, bladder; $2 \mathrm{cc}, 0.1 \mathrm{cc}$ ) were documented.

Results: The mean volumes for the different CTVs were measured: CTV_HR $48.3 \mathrm{ccm}$ (range 8-121 ccm), CTV_CT $61.5 \mathrm{ccm}$ (range 15-131 ccm), CTV_MR $51.3 \mathrm{ccm}$ (range 10-125 ccm). The treated volumes (CTV_HR) were similar to the measured CTV_MR volumes. But they deviated significantly from the CTV_CT volumes. As a result, the estimated dose constraints for the OaRs, using the CTV_CT volumes, were all larger. Using the three different volumes (CTV_HR, _CT, _MR) to create the treatment plans, all dose volume parameters and constraints (i. a. V100, D90, D98; COIN, DNR) could be achieved. Conclusion: MRI-assisted brachytherapy contouring allows for better protection of the OaRs. Especially for younger inexperienced colleagues the additional MRI represent a great benefit. Hereafter all free hand implants as well as the 1st intrauterine HDR-BT applications will receive an additional post implantation MRI to achieve a better protection of the normal tissue and OaRs.

\section{Unexpected severe optic pathway toxicity in a patient with meningioma treated with pencil-beam scanning proton therapy: a case report \\ Type: Physics}

Presenting Author:

A. Bolsi

\section{Institutions:}

Paul Scherrer Institute, Villigen

Department:

Center for Proton Therapy

Aims: We report on a case of a patient treated with pencil-beam-scanning proton therapy (PBS-PT) who developed severe optic pathway toxicity despite all optical structures dose constraints being within well-established limits.

Methods: The otherwise healthy 50-year-old woman was treated with definitive PBS-PT for a skull-base meningioma with no ophthalmologic impairment at treatment initiation. The treatment was delivered in a single plan using three (two quasi-lateral and a vertex) fields up to 50.4
$\mathrm{Gy}(\mathrm{RBE})$ at $1.8 \mathrm{~Gy}(\mathrm{RBE}) /$ fraction. Nominal doses to optical structures were below $52 \mathrm{~Gy}(\mathrm{RBE})$ (Dmax) and $51 \mathrm{~Gy}(\mathrm{RBE})$ (D2\%).

Results: Five months after treatment, the patient presented with visual field defect on the right eye, quickly deteriorating to amaurosis, followed shortly afterwards by visual field defect on the left eye. A diagnosis of radiation-induced optic neuropathy was confirmed on MRI, which showed inflammatory changes in both chiasmatic and proximal optic nerve regions.

Consequently, a comprehensive treatment review was performed, including nominal and log-file reconstructed dose distributions, delivery accuracy (patient positioning, machine QA, log file calculation), plan robustness and LET distribution. As with the nominal dose distributions, log-file reconstructed doses resulted in low-risk dose levels in the optic structures (Table 1), and MR changes were in low LET $(<3 \mathrm{KeV} / \mu \mathrm{m})$ areas. Patient positioning was within the typical accuracy of PSI patients with bite block immobilization (1.62 mm residual random error). Robustness analysis, including fractionation, resulted in Max/D2 doses below 54 Gy(RBE) for all optical structures (Table 1).

Conclusion: After this comprehensive review, the observed toxicity could not be correlated to any dosimetric, delivery or LET based metrics. This may demonstrate that risk of toxicity is a complex, multi-variate problem also involving patient specific factors, and not just the oft-discussed issues of end of range LET/RBE and robustness.

\section{Generating clinical benefit from competition winning tomotherapy plans \\ Type: Physics}

Presenting Author:

Simon Heinze

Authors:

S. Heinze, K. Buchauer, L. Plasswilm, H. Schiefer

Institutions:

Kantonsspital St. Gallen

Department:

Klinik für Radio-Oncology

Aims: The first purpose of the this work is to determine whether simple rules can be drawn from competition winning Tomotherapy plans in order to achieve plans with similar quality in clinical practice. Secondly, to ensure that the generated test plans with increasing complexity are still well deliverable, film measurements are performed. The generated plans are compared with originally submitted plans of the planning competition.

Methods: A spine SBRT re-irradiation competition case of the recent Accuray PLANit Global Treatment Planning Challenge (Accuray,

Table 1

\begin{tabular}{lllll}
\hline Dose distribution & Nominal & \multicolumn{3}{l}{ Log file based } \\
\hline & Max $(G y(R B E))$ & $D 2 \%(G y R B E)$ & $\operatorname{Max}(G y(R B E))$ & $D 2 \%(G y(R B E))$ \\
Optic Nerve R & 51.3 & 50.7 & 51.3 & 50.5 \\
Optic Nerve L & 51.6 & 50.9 & 50.6 & 50.3 \\
Chiasma & 50.8 & 50.6 & 50.4 & 50.1 \\
MRI changes ONR & 51.4 & 50.9 & 50.9 & 50.6 \\
MRI changes ONL & 51.4 & 51.2 & 50.8 & Worst case fractionation \\
\hline Error distribution & Worst case no fractionation & Max $(G y(R B E))$ & \\
\hline & Max $(G y(R B E))$ & & 54.0 & 53.4 \\
Optic Nerve R & 58.4 & & 51.0 & 53.0 \\
Optic Nerve L & 58.9 & & 53.3 \\
Chiasma & 53.4 & &
\end{tabular}


USA) is used to generate two sets of seven test plans. 10 to 4000 iterations are used to obtain plans with different degrees of complexity. The ProKnow scoring system (ProKnow, USA) is used to obtain a clinical quality score for the plans. The first set is based on the original clinical goals of the competition as planning input with equally weighted constrains. The second set of plans is generated with the more sophisticated weighting and importance settings of the competition best Tomotherapy plan. Film dosimetry (Ashland Inc., USA) is used for evaluation of deliverability.

Results: ProKnow scoring of the plans was found to be positively correlated to the number of iterations. With 200 iterations, the first set yielded a plan scoring in the median of all plans originally submitted to the competition. With the more sophisticated constraints and using 200 Iterations, it was possible to achieve a plan that would have scored third in the competition. Gamma pass rates were $98.7 \pm 0.8$ with no correlation to plan scoring, respectively plan complexity.

Conclusion: Half of all competition plans are rated worse than plans based on the simplest constrains, underscoring the importance of this work: Make adjustments in a gentle and meaningful way. The film measurements demonstrate that complex plans can be applied without dosimetric degradation, meaning complex plans transfer into real benefit for the patient.

Towards FLASH proton therapy: achievable dose rates in geometrical and clinical fields for pencil beam scanning Type: Physics

\section{Presenting Author:}

Sairos Safai

Authors:

Sairos Safai, Steven van de Water, Damien C. Weber,

Antony J. Lomax

\section{Institutions:}

Paul Scherrer Institute, Villigen

Department:

Center for Proton Therapy

Aim: to investigate the performance of ionization chambers when exposed to proton dose rates in the lower end of the FLASH dose rate (>30 Gy/s) for pencil beam scanning (PBS) and to investigate achievable dose rates in geometrical and clinical fields for a commercial PBS gantry.

Methods: Achievable instantaneous dose rates on the central pencil beam axis (within a pulse and the mean rate) for a clinical Varian ProBeam system were estimated based on the cyclotron repetition frequency, pulse width, proton current at isocenter, pencil beam dose-area-product and pencil beam size in water. Dose measurements were performed with a Farmer ionization chamber in the middle of a $10 \times 10 \times 5 \mathrm{~cm}^{3}$ SOBP at different dose rates and dose levels. To quantify dose rates in three-dimensions for clinical fields, we proposed the 'dose-averaged dose rate' (DADR) metric, defined for each voxel as the dose-weighted mean of the instantaneous dose rates of all spots. This concept was applied to four head-and-neck cases, each planned with clinical (4 fields) and various spot-reduced IMPT techniques. DADR distributions were calculated assuming 2 or 6 Gy fractions.

Results: During PBS, currents around $5 \mathrm{nA}$ at isocenter could be reached. An individual pencil beam could deliver up to $120 \mathrm{~Gy} / \mathrm{s}$ in water. When the Farmer chamber was exposed to up to $100 \mathrm{~Gy} / \mathrm{s}$ and $8 \mathrm{~Gy}$, no significant dose rate dependence was observed. For clinical plans, the mean DADR in normal tissue for a 2 Gy fraction was $1.7 \mathrm{~Gy} / \mathrm{s}$ (median over all patients) at maximum, whereas in standard spot-reduced plans it was $4.4 \mathrm{~Gy} / \mathrm{s}$. Arc-shoot-through planning resulted in DADR values of $6.0 \mathrm{~Gy} / \mathrm{s}$. Hypofractionation $(3 \mathrm{x})$ resulted in higher dose rates, up to $73.2 \mathrm{~Gy} / \mathrm{s}$ for arc-shoot-through plans.

Conclusion: FLASH dose rates can be delivered and measured for PBS proton therapy under experimental conditions. However, FLASH dose rates cannot be achieved under clinical conditions without increasing spot-wise beam intensities and the use of spot-reduction, hypofractionation, and arc-shoot-through delivery.

\section{Evaluation of $\mathrm{iCBCT}$, a new CBCT iterative reconstruction algorithm: potential imaging dose reduction and evaluation of dose calculation accuracy in the pelvic area \\ Type: Physics}

Presenting Author:

Gregory Bolard

Institutions:

La Tour Medical Group

Department:

Radiation oncology

Varian released recently iCBCT, a new iterative reconstruction algorithm, for head and pelvic anatomies. Phantom studies show that iCBCT exhibits better image uniformity and a superior low contrast detectability in comparison to standard reconstruction. The first objective of this study was to estimate the dose reduction achievable in order to solve the minimal contrast discriminating organs in the pelvic area $(2 \%)$. The second aim was the evaluation of photon beam dose calculation accuracy on these iCBCT datasets. The last purpose was to assess the stability with time of several imaging performance parameters and the constancy of the HU to mass density curve.

Material and methods: The low contrast and uniformity sections of the Catphan 604 phantom were used to optimize the default manufacturer-provided pelvis protocol. The difference of mean HU between peripherical ROI and a central ROI was recorded to score image uniformity. The low contrast detectability was estimated using a signal to background approach calibrated against operator observation. Imaging dose was iteratively reduced until the low contrast detectability was not satisfactory anymore. The HU to mass density curve was measured using a CIRS062 phantom. To check the reliability of dose calculation, 13 pelvic VMAT plans were recalculated (Philips Pinnacle3) on CBCT acquired at the 1 st fraction and the dose distributions were compared to the original plan using different metrics (CTV mean dose, 3D gamma 1\%, $1 \mathrm{~mm}$ ).

Results: Using a smooth filter and a reduced acquisition frame rate, an imaging dose reduction by a factor five is feasible to solve $1 \%$ contrast (CTDI $_{\mathrm{vol}}$ of $3.7 \mathrm{mGy}$ ). The noise is kept at a low value with a mean standard deviation of $2.4 \mathrm{HU}$. The uniformity is acceptable with a maximum HU difference of $20 \mathrm{HU}$ in a $30 \mathrm{~cm}$ diameter object. Dose calculation comparison is satisfactory with a CTV mean dose difference less than $1 \%$ and a 3D gamma pass rate greater than $95 \%$ for all cases. The HU to mass density relationship is stable in time with maximum mass density differences of $0.1 \mathrm{~g} / \mathrm{cc}$.

Conclusion: iCBCT enables clinically acceptable dose calculation accuracy with a reduced exposure for pelvis scans.

\section{Is volumetric modulated arc treatment (VMAT) a robust irradiation technique for the breast/thoracic wall? Type: Physics}

\section{Presenting Authors:}

Tino Streller, David Jeller, Thomas Götzfried, Paolo Zucchetti, Philippe Logaritsch, Regina Seiler

\section{Institutions: \\ Luzerner Kantonsspital \\ Department: \\ Radio-Onkologie}

Aim: To evaluate the VMAT technique for breast/thoracic wall (TW) irradiation with respect to uncertainties due to breathing motion.

Methods: 10 patients with breast or TW irradiation (40 Gy/15 fractions), including the axillary and internal mammary lymph nodes, were 
included in this study. All patients were treated with 4 VMAT arcs, 6 with free breathing (FB) and 4 with deep inspiration breath hold (DIBH). The VMAT plans were made with a virtual bolus to ensure that $>90 \%$ of the CTV is covered with 38 Gy (95\% prescription dose), taking potential translational isocenter shifts of up to $5 \mathrm{~mm}$ into account. During treatment, the body surface motion signal of the breast/ TW was recorded. Beam-on was allowed when the FB signal was lower than $5 \mathrm{~mm}$ or the DIBH signal was $+/-2 \mathrm{~mm}$ from the reference level. During each fraction, tangential MV images of the breast/TW were acquired between the 2nd and 3rd VMAT arc. For each image, the maximal surface difference (MSD) between actual and planned breast/ TW body surface position was measured.

Results: 135 tangential MV images were analyzed. The mean absolute MSD was $2.7 \pm 1.8 \mathrm{~mm}$. Absolute MSD values $>5 \mathrm{~mm}$ were found in 13 images (FB) and 3 images (DIBH). For two of the FB patients, 5 images were found with MSD $>5 \mathrm{~mm}$, whereas for all other patients there were not more than 2 images with MSD $>5 \mathrm{~mm}$.

Conclusion: VMAT plans for breast/TW irradiation may be reproducibly applied both with FB and DIBH, but regular tangential imaging is necessary to avoid individual systematic misalignment of the breast/ TW surface.

\section{The combination of A-966492 and topotecan for effective radiosensitization on glioblastoma spheroids Type: Physics}

Presenting Author:

Fereshteh Koosha

\section{Institutions:}

Shahid Beheshti University of Medical Sciences, Tehran

Department:

Radiation Technology

Radiotherapy is one of the modalities in the treatment of glioblastoma patients, but glioma tumors are resistant to radiation and also chemotherapy drugs. Thus, researchers are investigating drugs which have radiosensitization capabilities in order to improve radiotherapy. PARP enzymes and topoisomerase I enzymes have a critical role in repairing DNA damage in tumor cells. Thus, inhibiting activity of these enzymes helps stop DNA damage repair and increase DSB lethal damages. In the current study, we investigated the combination of TPT as a topoisomerase I inhibitor, and A-966492 as a novel PARP inhibitor for further radiosensitization.

U87MG cells: (a human glioblastoma cell line) were cultured in Poly-Hema coated flasks to reach $300 \mu \mathrm{m}$-diameter spheroids. Treatments were accomplished by using non-toxic concentrations of A-966492 and Topotecan. The surviving fraction of treated cells was determined by clonogenic assay after treatment with drugs and $6 \mathrm{MV}$ $\mathrm{X}$-ray. The $\gamma$-H2AX expression was measured by an immunofluorescence staining method to examine the influence of A-966492, TPT and radiation on the induction of double stranded DNA breaks. Treatments using the A-966492 drug were conducted in concentration of $1 \mu \mathrm{M}$. Combining A-966492 and TPT with radiation yielded enhanced cell killing, as demonstrated by a sensitizer enhancement ratio at $50 \%$ survival (SER50) 1.39 and 1.16 respectively. Radio- and chemo-sensitization was further enhanced when A-966492 was combined with both $\mathrm{X}$-ray and TPT, with SER50 of 1.53 .

Also $\gamma$ : - H2AX expression was higher in the group treated with a combination of drugs and radiation.

A-966492 is an effective PARP inhibitor and has significant radio-sensitivity on U87MG spheroids. By accumulating cells in the S phase and by inhibiting the DNA damage repair, TPT enhanced radio-sensitivity. A-966492 combined with TPT as a topoisomerase I inhibitor had additive radio-sensitizing effects. As a result, applying PARP and topoisomerase I inhibitors can be a suitable strategy for improving radiotherapy in clinics.

\section{Type: Biology}

\section{Does hyperthermia clinically alter the $\alpha / \beta$ ? Insights from thermoradiotherapy vs. radiotherapy trials \\ Type: Biology}

\section{Presenting Author:}

Niloy Ranjan Datta

Authors:

N.R. Datta, S. Bodis

Institutions:

Kantonsspital Aarau, Kantonsspital Baden

Department:

Radiation Oncology

Introduction: Hyperthermia at $39-43^{\circ} \mathrm{C}$ is a known to inhibit post-radiation repair of the sublethal and potentially lethal DNA damage. This should be expected to alter the $\alpha / \beta$ values of the linear-quadratic (LQ) model. The present study has been carried out to clinically estimate the tumour $\alpha / \beta$ from studies of thermoradiotherapy (HTRT) vs. radiotherapy (RT).

Methods: 3 recently published meta-analyses for HTRT vs. RT in recurrent breast (RcBC), head and neck (stages III/IV) (LAHNC) and cervix cancers (stages IIB-IVA) (LACC) were evaluated for their complete response (CR). The biological effective dose with RT $\left(B D_{R T}\right)$ in these studies were individually computed using the $\mathrm{L}-\mathrm{Q}$ equation assuming an $\alpha / \beta_{\mathrm{RT}}$ of $10 \mathrm{~Gy}$ for all sites. Since all trials had a positive outcome with HTRT over RT, the resultant enhanced thermoradiobiological BED for HTRT $\left(\mathrm{BED}_{\text {HTRT }}\right)$ was correspondingly computed as a product of the $\mathrm{BED}_{\mathrm{RT}}$ and \%difference in CR between HTRT and RT. With the "D" and "d" of both HTRT and RT arms in each trial being similar, the $\alpha / \beta$ following HTRT ( $\alpha / \beta_{\text {HTRT }}$ ) was estimated as, $\alpha /$ $\beta_{\text {HTRT }}=\mathrm{Dd} /\left(\mathrm{BED}_{\mathrm{HTRT}}-\mathrm{D}\right)$, as per the L-Q model.

Results: 12 studies with 864 patients were shortlisted-RcBC (3 studies, $n=259$ ), LAHNC (5 studies, $n=338$ ) and LACC (4 studies, $n=267)$. Mean RT dose was 53.4 Gy (SD: \pm 14.3 ) delivered in $2.2 \mathrm{~Gy} /$ fraction (SD: \pm 0.74 ). The overall risk difference of 0.28 was in favour of HTRT $(p<0.001)$. Mean BED-RT was 64.7 Gy (SD: \pm 15.5$)$ while the mean BED-HTRT was estimated as 109.5 Gy (SD: \pm 32.1 ). The overall computed $\alpha / \beta_{\text {HTRT }}$ was 2.25 Gy (SD: \pm 0.79 ). The $\alpha / \beta_{\text {HTRT }}$ for RcBC, LAHNC and LACC were 2.05 Gy, 1.74 Gy and 3.03 Gy $(p=0.027)$, reflecting a reduction in $\alpha / \beta$ by HTRT (range: $69.7-82 \%$ ). On meta-regression, $\alpha / \beta_{\text {HTRT }}$ was the sole predictor for the corresponding risk differences of the studies (coefficient $=-0.096 ; p=0.03$ ).

Conclusion: This is the first reported clinical estimates of tumour $\alpha /$ $\beta_{\text {HTRT }}$ and mirrors invitro studies. The fall in $\alpha / \beta_{\text {HTRT }}$ could be due to reduced repair of RT induced DNA damage by HT. This has direct implications for effectively optimizing HTRT dose-fractionation schedules in the clinics.

\section{Screening for synthetic interactions in CHK2-deficient cancers} Type: Biology

\section{Presenting Author:}

Carmen Muñoz-Maldonado

Authors:

Carmen Muñoz-Maldonado, Michaela Poliaková,

Daniel M. Aebersold, Yitzhak Zimmer, Michaela Medová

\section{Institutions:}

Inselspital, Bern University Hospital

Department:

Department of Radiation Oncology

Aims: CHK2 is a protein of the DNA damage response cascade. After DNA damage, CHK2 arrests cell cycle at checkpoints or triggers apoptosis in presence of irreparable damage. Hence, CHK2 is essential 
for cell cycle regulation and its abnormal activity may be involved in cancer development. $\mathrm{CHK} 2$ mutations have been correlated with many types of cancers and associated with resistance to anthracycline-based chemotherapy in breast cancer patients. To find selective treatments for cancer therapy, the idea of synthetic lethality has been exploited. Two genes are in a synthetic lethal interaction when the loss of either gene alone does not impair cell viability but the perturbation of both genes is lethal. Based on this knowledge, our main aim is the identification of synthetic lethal partners of $\mathrm{CHK} 2$-deficient cancers.

Methods: For the identification of synthetic lethal partners of CHK2, a differential viability screening with a library of 400 non-selective, ATP competitive kinase inhibitors was performed using isogenic human colorectal cancer cell lines HCT116 proficient or deficient for CHK2. Findings of this screening were further studied by cell-based assays, Western blotting and qPCR.

Results: The screening with the library of kinase inhibitors revealed seven kinases as potential synthetic lethal partners of $C H K 2$-deficient cancers. Further analyses employing siRNA-mediated knockdowns and specific pharmacological inhibitors identify several hits as plausible CHK2 synthetic lethal partners.

Conclusion: CHK2 loss of function could affect tumors with susceptibility to specific drug interventions. The current study focuses on a pharmacologic screening of a kinase inhibitors library for the identification of CHK2 partners. Current data describes few candidate kinases as CHK2 partners whose targeting could serve as a plausible treatment strategy in $C H K 2$-deficient cancers.

\section{Fractionated synchrotron microbeam radiation therapy at FLASH dose-rates promotes tumor remission in mouse melanoma \\ Type: Biology}

Presenting Author:

Dr. Cristian Fernandez-Palomo

Authors:

Fernandez-Palomo C, Trappetti V, Djonov V

\section{Institutions:}

Institute of Anatomy, University of Bern

Department:

Faculty of Medicine

Introduction: Synchrotron Microbeam Radiation Therapy (S-MRT) provides excellent tumor control in several pre-clinical cancer models because it can deposit very high doses of radiation into the tumors. The remarkable dose-volume effect achieved by the S-MRT configuration allows delivering average peak-doses of $400 \mathrm{~Gy}$ at FLASH dose-rates of up to $17,000 \mathrm{~Gy} / \mathrm{s}$; with no radiation toxicity nor side-effects. In this context, we would like to report that temporally fractionating S-MRT had the unique capability of eradicating $50 \%$ of the primary melanomas of this study.

Methods: At the European Synchrotron, three-consecutive S-MRT sessions were delivered to mice harboring B16-F10 melanomas in both ears. The beam array of the S-MRT was composed of micro-planar beams of synchrotron x-rays 50 um wide, which were spaced by 200um. The dose-per-fraction of the S-MRT configuration was $133 \mathrm{~Gy}$ on the microbeams (peak dose) and $2 \mathrm{~Gy}$ in the gaps between them (valley-dose); the dose rate was 13,800 Gy/sec. Daily measurements of the tumor size provided the treatment effectivity.

Results: Tumors exposed to three daily S-MRT sessions of $133 \mathrm{~Gy}$ showed three distinct behaviors: i) non-responsive tumors (12.5\%), ii) responsive tumors $(37.5 \%)$ showing a growth delay was 12.8 days, and iii) tumor remission $(50 \%)$. The mice from the latter group were sacrificed 18 months after the treatment for further studies. Organ metastases were not found, and on the site of the melanoma, only a small nevus remained visible. Histopathological evaluation revealed that the nevus was formed entirely by melanophages
Conclusion: Fractionated S-MRT has the potential of inducing complete tumor remission and prevent organ metastases, which is essential for future clinical application of S-MRT due to the option of delivering lower peak-doses by temporal fractionation. Future efforts should focus on combining fractionated S-MRT with adjuvant therapies to improve its effectivity.

\section{Type: Technicians}

\section{CyberHeart: Lausanne experience}

Type: Technicians

Presenting Author:

Sebastiao, Laura

Institutions:

Centre Hospitalier Universitaire Vaudois

Department:

Oncologie

Aim: Stereotactic Radiotherapy (SBRT) is a technology widely used in oncology to treat, in a non-invasive manner, solid tumours with precision and efficiently. Recently, this technology has been evaluated in the treatment of ventricular tachycardia (TV).

The underlying principles, the concept and the results of the first clinical studies, which have created much interest for this therapeutic approach, will be described. The recent experimental treatment of patients suffering from refractory TV carried out at the Centre Hospitalier Universitaire Vaudois (CHUV) will be presented.

Method: In September 2017 the CHUV put into place a program of ablation by stereotactic radiotherapy for patients with TV resistant to medication and ablation by catheter. This program was approved by the CHUV ethical committee as part of individual experimental therapies.

Up until October 2018, 5 patients with refractory TV were treated by SBRT with a Cyberknife ${ }^{\circledR}$ (Accuray, Sunnyvale, CA) fitted with a continuous imaging and tracking system. The localization of the arrhythmogenic substrate was established using cardiac imaging and three-dimensional electro-anatomical mapping.

Results: The primary criteria of effectiveness were the reduction in number of TV episodes and the number of shocks from the implanted defibrillator after the treatment. In all the cases these numbers decreased.

The treatment was well tolerated and no radio-induced toxicity was described during the follow-up, on average 7 months after treatment (6-11 months)

Conclusions: Stereotaxic Radiotherapy is a promising treatment for refractory TV patients.

A longer follow-up period of treated patients as well as the results of future studies will allow a democratization of the technique.

New techniques using image guided RT are emerging, especially the MRI Linac which could provide, in the near future, the possibility to combine non-irradiating imaging with non-invasive tracking.

Initial clinical experience with the MR-Linac system - treatment workflow management

Type: Technicians

Presenting Author:

Amanda Moreira

Authors:

A. Moreira, N. Weitkamp, N. Andratschke, M. Guckenberger,

H. I. Garcia Schüler, S. Tanadini-Lang

Institutions:

University Hospital Zurich

Department:

Department of Radiation Oncology 
Objective: With the MRIdian system (Viewray) it is now possible to image radiotherapy patients using MRI technology not only for initial positioning but also during beam delivery. The treatment plan can be adapted to accommodate daily variations in patient anatomy, although this process prolongs the time a patient lies in the treatment position. This study aimed at evaluating the time efficiency of a fully adaptive workflow.

Materials and methods: 8 patients with 32 fractions were treated with the MRidian. Daily procedure includes initial patient set up, low-resolution followed by high-resolution MR imaging, re-contouring, plan re-optimization, QA procedure, re-imaging, a check of gating feasibility if beam delivery is to be gated and finally beam delivery. Patients were scheduled for a $90 \mathrm{~min}$ appointment for their first fraction and 60 min subsequently. The time required for each step of the treatment process was recorded and analyzed. Total treatment time was defined as the patient's total time on the treatment table.

Results: Median total treatment time after 1 month of machine use, was $69 \pm 7 \mathrm{~min}$ for the first fraction and $57 \pm 13$ for subsequent fractions. Contouring has the highest median time of $9 \mathrm{~min}$. Contouring time is heavily dependent on treatment site ( 4 min in lung, 12 in abdomen). Time needed for other processes was not dependent on treatment site. Median time was $6 \pm 1 \mathrm{~min}$ for in room set up, $9 \pm 3$ for total image matching (acquisition and match time for both low and high-resolution images), $9 \pm 5$ for contouring, $7 \pm 3$ for optimization and $4 \pm 1,8 \pm 3$, and $10 \pm 2$ min for QA, gating feasibility check and beam time respectively after 1 month.

Conclusion: Through proper staff training and preparation a patient can be treated with a fully daily adaptive approach in less than $60 \mathrm{~min}$.

\section{Implementation of respiratory coaching in the management of left breast cancer using the deep inspiration breath hold technique \\ Type: Technicians}

Presenting Author:

Laurent Marmy

Authors:

L. Marmy ${ }^{1,2}$, N. Missègue ${ }^{1}$, G. Risse ${ }^{1}$, A. S. Allal ${ }^{1}$

\section{Institutions:}

${ }^{1}$ HFR-Fribourg Hospital, ${ }^{2}$ HESAV, Haute Ecole de Santé Vaud, Lausanne

Department:

${ }^{1}$ Radiation Oncology Department

Objectives: The main objective of implementing a specific RTT preparatory coaching is to optimize simulation preparation and first treatment sessions for patients with left breast cancer treated with the deep inspiration breath hold technique.

Method: The implementation project was based on the Ottawa model. We identified several interventions that can be carried out for this implementation, including theoretical and practical training of professionals based on the concept of motivational interview. The literature review highlighted the importance of respiratory coaching. Patient management has been proved to potentially reduce side effects by protecting organs at risk such as the heart and the lungs.

Results: This interview will allow us to collect information on patient positioning and preparation before the CT-scanning and thus better anticipate possible difficulties (pain, reduced mobility, respiratory problems, stress and anxiety). It will also help reduce anxiety and avoid sub-optimal inspiration. The effects of the intervention will be first evaluated by a quantitative study (pre-post) by measuring the following indicators: number of imperfect treatments ("failures") during the simulation and the first days of treatment and machine occupancy time (Computed tomography simulation and daily treatment). Patient and professional satisfaction will be evaluated later on.
Conclusion: The aim of respiratory coaching by RTTs is to improve patient management in terms of quality of radiotherapy preparation and treatment delivery conditions, while strengthening the relationship of RTTs with patients with left breast cancer treated with the DIBH technique. Success in the implementation of this procedure for left brest cancer will open opportunities for other sublocated tumors.

\section{Use of pseudo CT for dose calculation of treatment plans on the viewray MRIdian linac}

Type: Technicians

\section{Presenting Author:}

Madalyne Chamberlain

Authors:

M Chamberlain, L Wilke, J Krayenbühl, H Garcia Schüler, S Ehrbar, M Bogowicz, N Andratschke, M Guckenberger, S Tanadini-Lang

\section{Institutions:}

University Hospital Zurich

Department:

Department of Radiation Oncology

Aim: MRI guided radiation therapy uses MR imaging for target definition and treatment setup, however, CT densities are still required for dose calculation. One option to achieve this is to perform a registration of a planning CT to the planning MRI and use the CT for dose calculation. Another option is to create a Pseudo CT. We compared the dose calculation using CT densities with a manually created Pseudo CT.

Method: For 10 patients planned with IMRT on the Viewray MRIdian MR Linac the dose calculation was performed using a registered planning CT for density information, and calculated with Monte Carlo. Additionally the MRIs were manually segmented into fat, water, lung, bone, and soft tissue and densities were assigned to these tissue types according to ICRU report 46. The plans were copied on to the Pseudo $\mathrm{CT}$ and dose calculation was repeated. Mean dose to the PTV, and to a nearby organ at risk were compared.

Results: The median PTV dose difference between the two density correction methods was $1.06 \%(0-4.46 \%)$ and the median difference in dose to the OAR was $0.7 \%(0.4-1.72 \%)$. In all cases, except one, the difference was less than $3 \%$ for both PTV and OAR. The two cases with larger deviations were lung cases. For these the density of the lung had to be lowered compared to the values of the ICRU 46 report because the patient was scanned in inspiration. Additionally a separate density had to be assigned to the GTV structure. With these adaptions, both lung cases had an agreement between the two techniques, which was within 5\%. Conclusion: Both density corrections methods show similar results. The registered CT method used as a standard in the commercial Software can be considered as safe. The Pseudo CT technique can be used for non-lung patients to replace additional CT Imaging.

\section{Different fixation methods for extracranial vertebral radiosurgery \\ Type: Technicians}

Presenting Author:

Shanna Roosenthaler

Authors:

Roosenthaler S., Mutsaers L., Lazeroms T., Rabe E., Bodis S.

Institutions:

Kantonsspital Aarau, Kantonsspital Baden

Department:

Radiotherapy, Radio-Onkologie-Zentrum

Aim: To find a more stable fixation for patients treated with SBRT for vertebral metastases in the neck region.

Method: Until now Kantonsspital Aarau (KSA) used a positioning method which includes a vacuum cast and a 5 point mask (Unger 
Medizintechnik, Germany, Mülheim-Kärlich). The positioning with this system can sometimes be unstable. Therefore a new head and neck support system was introduced in KSA, the customizable head support (CHS) from CIVCO Solumedics (Solumedics AG, Aarau, Switzerland). A limited number of patients will be treated with this CHS using the same 5 point mask during the test phase. 14 patients treated with the old positioning method will be retrospectively evaluated, with the pitch, roll and rotation analysed as these are the most critical directions to correct. All patients received a CBCT or KVKV 3D before the first 3 fractions and these data were used to analyze the pitch, roll and rotation of the patient. The CBCTs were made with a Varian Truebeam linac. The pitch, roll and rotation were matched in the offline matching environment in aria, in degrees $\left(^{\circ}\right)$.

Results: So far only one patient has been treated using the new fixation. With the old fixation, the overall pitch for the 14 patients was $0.6^{\circ}$, the overall roll was $0.7^{\circ}$ and the overall rotation was $0.6^{\circ}$. For the one patient treated with the new fixation system these values were respectively $0^{\circ}, 0.1^{\circ}$ and $0.3^{\circ}$.

Conclusion: The preliminary conclusion for this small study would be that the new fixation method using the CHS is promising. Results of a larger patient cohort with the next fixation method will be presented at SASRO. Final results of a larger patient cohort treated with the CHS may be needed to formulate a firm conclusion.

\section{Quality manager. a new job in radiotherapy \\ Type: Technicians}

Presenting Author:

Quentin Arnould-Durst

\section{Institutions:}

Hôpital du Valais, Sion

Department:

Radio-oncologie

Aims: Clinical audits of radiotherapy practices is a quality improvement process that seeks to improve patient care and outcomes through systematic review of care against explicit criteria and the implementation of change. To achieve these goals the audited institution must prepare a quality handbook, containing notably quality management documents and continuous improvement processes. Developing and maintaining a quality handbook is a time consuming task that requires appropriate skills. The poster describes the appointment of a quality manager for the department of radiotherapy in Sion in April 2019, his tasks and the first results obtained.

Methods: The first step is to define clearly the tasks of the quality manager. In our case, collection, update and writing of the missing quality documents, reactive analysis of incidents and improvement proposition, proactive risk assessment in the form of failure mode and effect analysis and lastly colleagues training. The second step is to convince the hospital management of the need and the advantages of having a dedicated quality manager. Amongst the arguments, were the time needed to perform all these mandatory tasks and the positive experience of many European centers.

Results: The lack of legal obligation made difficult to prove the need of having a dedicated quality manager. Eventually a $20 \%$ full time equivalent RTT position could be appointed and the work started in April 2019. At the present time (May 2019) the quality manager works on the collection of the quality documents and the writing of the most urgently lacking ones. He is also ready to take over with the critical incidents analysis. The failure mode and effect analysis is left for a later time.

Conclusion: With the appointment of a dedicated quality manager with a RTT background, the department of radiotherapy in Sion has the ambition to have in a near future a complete, homogeneous and upto-date quality handbook
Hippocampus sparing whole brain radiotherapy planning with a biologic model

Type: Technicians

Presenting Author:

Michael Iff

Authors:

M. Iff, J. Krayenbuehl, M. Guckenberger, L. Wilke, S. Tanadini-

Lang, N. Andratschke

Institutions:

University Hospital Zurich

Department:

Department of Radiation Oncology

Purpose: Whole-brain radiotherapy (WBRT) using 2D or 3D planning has been standard for patients with multiple metastases for decades. The introduction of new radiation technique, such as intensity modulated radiotherapy allows hippocampus sparing whole brain radiotherapy (HS-WBRT). This new approach aims to decrease the adverse neurocognitive effects of WBRT (RTOG 0933). Additionally, treatment planning system introduced biological models for dose optimization taking into account the type of organs irradiated (serial or parallel organ for example). In this study, a treatment planning model using biological constraints was evaluated for the treatment of HS-WBRT.

Methods and materials: Five randomly selected patients with HSWBRT with a dose fractionation of $10 \times 3$ Gy or $10 \times 2.5$ Gy where re-optimized in Eclipse (V. 15.6, Varian Medical System, Palo Alto, CA) using the general equivalent dose (gEUD). A total of four volumetric modulated arcs per plan with two table rotations were used. Plans were normalized to $92 \%$ of the target volume receiving the prescribed dose. The constraints used for the organ at risks where as recommended by the RTOG 0933 working group.

Results: All patients optimized with our template fulfilled the clinical planning objectives. The minimal mean dose to the left and right hippocampus was $8.6 \mathrm{~Gy}$. The mean total hippocampus dose was 10.4 Gy and the maximal dose point dose to the hippocampus was $15.9 \mathrm{~Gy}$. The mean maximal brain dose, defined as the dose to $2 \%$ of the target volume, was $33.8 \mathrm{~Gy}$, substantially lower than recommended in the RTOG, 37.5 Gy.

The median planning time for all patient was $30 \mathrm{~min}$.

Conclusion: The HS-WBRT model using a biological model fulfilled all the recommendations from the RTOG 0933 study while significantly improving dose homogeneity and decreasing unnecessary hot spots in the normal brain.

\section{Function of carbon transfer table for bedridden patients} Type: Technicians

Presenting Author:

Audrey Hendricks

Authors:

A. Hendricks, C. Liang, Dr. med. U. Meier, J. Gomes

Institutions:

Kantonsspital Winterthur

Department:

Radio-Onkologie

Aims: Transferring bedridden patients from a hospital bed to a radiation treatment table can be time consuming and tasking for employees and patients. In order to safely transfer a bedridden patient for palliative treatment, a minimum of four people are required to carry out the time consuming procedure. Despite prior scheduling and proper transfer technique, problems often arise regarding employee safety, patient comfort, and time efficiency. With this in mind, the following question was established: How can we more efficiently transfer bedridden pa- 
tients to save time, increase patient comfort and improve employee safety?

Methods: HESS Medizintechnik AG was approached to develop a Radiation Therapy specific transfer table for bedridden patients. The following requirements were given for production: ability to position the patient onto the transfer table outside of the treatment room, transferring the patient from said table onto the linear accelerator table without further movement of the patient, and the ability to treat through the transfer table. The solution from HESS was a mobile, detachable radiolucent lying surface. This new Transfer Table is carbon made and ensured by various radiation tests and designed to perfectly lay over the linear accelerator table.

Results: The Carbon Transfer Table was first used between the 19th and 26th of March. The analysis was based on this period.

Conclusions: The Carbon Transfer Table was effective in reducing the amount of time spent with the patient in the room. It became possible to keep the patient in a 10-15 Minute time slot. In combination with a patient transfer sheet, only two staff members were needed to transfer the patient to the carbon transfer table and subsequently to the radiation treatment table. In addition, transfers were smoother and thus led to less patient and staff complaints. The carbon transfer table was successful in saving time, increasing patient comfort, and employee safety.

\section{The effectiveness of PerfectPitch 6 degrees of freedom couch in patient set-up accuracy and PTV coverage \\ Type: Technicians}

Presenting Author:

Audrey Hendricks

Authors:

A. Hendricks, A. Luu, C. Pires, K. Alves, J. Gomes, H. Härle

\section{Institutions:}

Kantonsspital Winterthur

Department:

Radio-Onkolgie

Aims: Even with the use of Civco IMRT Reinforced Thermoplastic masks, there is still difficulty in accurately positioning head and neck patients. Often as a patient loses weight, it becomes more difficult to reproduce the same patient position. With the use of Varian medical systems PerfectPitch 6 degrees of freedom couch (6DoF), new possibilities regarding patient set-up accuracy have arisen. To help determine how the $6 \mathrm{DoF}$ can best be utilized, the following questions were determined: Is using 6DoF effective in helping increase patient set-up accuracy? Is there a big difference in PTV coverage with or without $6 \mathrm{DoF}$ ? Methods: A new protocol was developed regarding patients with a Head, Neck and Shoulder Thermoplastic Mask and CBCT imaging. Each patient was scheduled for imaging the first three days and then once per week. More imaging was done as necessary. To determine the difference between using $6 \mathrm{DoF}$ and not, each patient was first auto matched without $6 \mathrm{DoF}$ and then auto matched with $6 \mathrm{DoF}$. Manual adjustments were applied per the technologist's discretion. All data was recorded for later analysis. An "optimal PTV" was created for each CBCT using deformable registration. The 95\% Isodose-coverage of this PTV was evaluated using Registrations with and without $6 \mathrm{DoF}$.

Results: $69.05 \%$ 6DoF registrations showed an improved coverage of the PTV for the $95 \%$ Isodose compared to non $6 \mathrm{DoF}$ registrations. Notably, $71 \%$ of the patient images had a rotation, pitch or roll greater than one degree.

Conclusions: The use of $6 \mathrm{DoF}$ was successful in helping correct patient positional errors. Subsequently, that reduced the need to re-position the patient manually. Further, the registration images revealed that we can improve our treatment accuracy and PTV coverage by using $6 \mathrm{DoF}$.
Therapeutic communication ... what else?

Type: Technicians

Presenting Author:

Mamboury Nicolas/Marmy Laurent

Institutions:

HESAV

Department:

Filière TRM

The universe of radio-oncology has undergone a deep technological evolution over the last decade. The technical platforms, as well as the different treatment modalities have become more complex both in terms of imagery than irradiation techniques; this discipline has become very attractive, and even fun for young technicians in radio-oncology.

Confronted to growing technology and medicine "style arts ménagers" according to some authors, the risk incurred by the patient in this particular context is a reification, relegated to the rank of an object or simple pathology to be irradiated.

The patient of a cancerous disease, weakened, rendered vulnerable, emotionally shaken and perhaps reached in its dignity requires a personalized and empathetic support.

"The patient at the Center" is the leitmotif in the mouth of many health care providers. But what does it mean in reality and how putting it into daily practice, while the economy, effectiveness and efficiency are required?

The famous American psychologist Carl Rogers laid the foundations for a person centered approach, empathy being an essential feature in the field of communication.

But a so-called empathetic communication can also be toxic or destructive depending on investment developed by one of the speakers or by the terms used.

This communication proposes a reflection on the modes of communication of the radiation oncology technician and the benefit to the patient.

Note: the epicene language is used to lighten the text.

\section{Type: Clinical}

Impact of patient and treatment characteristics on heart and lung dose in adjuvant radiotherapy for left-sided breast cancer Type: Clinical

\section{Presenting Author: \\ Van Trinh Nguyen}

\section{Institutions:}

University Hospital Basel

Department:

Clinic of Radiotherapy and Radiation Oncology

Purpose: The heart and lungs are routinely exposed to incidental irradiation during adjuvant radiotherapy (RT) of breast cancer. We analyzed the impact of patient and treatment factors on heart and lung dose in left-sided breast RT.

Methods: We analyzed 344 female patients treated with left-sided breast RT in our institution between 2013 and 2018. Mean heart dose (MHD), left mean lung dose (MLD) and heart/lung V20Gy were collected from treatment plans. Patients were stratified by RT technique (3D-conformal RT, 3DCRT; intensity-modulated RT, IMRT; volumetric modulated arc therapy, VMAT) and target volumes, including lymph node RT (LN-RT). Patient characteristics (body mass index (BMI), heart and lung volume) were assessed using correlation analyses. 
Results: LN-RT was performed in 111 patients and increased MHD (median 4.6 vs. $3.3 \mathrm{~Gy} ; p<0.01$ ), left MLD (14.8 vs. $7.7 \mathrm{~Gy} ; p<0.01$ ) and left lung V20Gy $(30.0 \%$ vs. $14.4 \%$; $p<0.01)$ compared to treatment without LN-RT. Internal mammary LN (IMN)-RT further increased organ doses compared to RT involving only supraclavicular $+/-$ axillary $\mathrm{LN}(p<0.01$ for all values; MHD 6.9 vs. 4.2 Gy). In 233 patients without LN-RT, IMRT/VMAT was associated with higher left lung doses (MLD 9.1 vs. $7.4 \mathrm{~Gy}, p<0.01$; V20Gy $18.0 \%$ vs. $14.0 \%$, $p<0.01)$ compared to 3DCRT. A negative correlation between total lung volume and both MHD $(\mathrm{r}=-0.38, p<0.01)$ and heart V20Gy $(\mathrm{r}=-0.36, p<0.01)$, as well as a weak positive correlation of BMI and MHD ( $\mathrm{r}=0.25, p<0.01)$ were observed.

Conclusions: In left-sided breast RT, LN-RT is associated with a marked increase in heart and lung doses, particularly with internal mammary LN-RT. In cases without LN-RT, higher lung doses with IMRT/VMAT may be caused by selecting this technique for patients with a challenging anatomy. Nevertheless, conceivable advantages of IMRT/VMAT for dose conformity/homogeneity need to be weighed against a potential, moderate increase in lung dose.

\section{Pineal parenchymal tumor of intermediate differentiation: experience of two cases \\ Type: Clinical}

Presenting Author:

Süveg K

Authors:

Süveg K, Brügge D, Plasswilm L

\section{Institutions:}

Kantonsspital St. Gallen

Department:

Klinik für Radioonkologie

Objective: Pineal parenchymal tumors (PPT) account for $<0.1-0.3 \%$ of all CNS tumors. In 2007, the WHO classification divided PPTs into pineocytoma (PC), pineoblastoma (PB) and PPT of intermediate differentation (PPTID). The mainstay of therapy in PPTID is surgery, the role of radiotherapy (RT) and chemotherapy is not clearly defined.

Material and methods: Between 2007-2018 two patients with PPTID $\mathrm{II}^{\circ}$ and $\mathrm{III}^{\circ}$ were treated at our department. In the first case, a 25-yearold man underwent external ventricular drainage (EVD) followed by tumor resection. The histopathological work-up including a second opinion (Neuropathology, Charité, Germany) confirmed the diagnosis of PPTID III ${ }^{\circ}$. MRI follow-up showed minimal residual disease in the resection cavity, which was progressive 3 months after resection. Additive RT was started 4 months after surgery. In the second case, a 75-year-old patient underwent subtotal resection of a pineal tumor with a residual mass of $8 \times 7 \mathrm{~mm}$ in the post-surgical MRI. The histopathological work-up revealed a $\mathrm{II}^{\circ}$ tumor which was confirmed by a second opinion. The patient underwent an additive RT 3 months post-surgery. In case of the $\mathrm{III}^{\circ}$ tumor we delivered $24 \mathrm{~Gy}(15 \times 1.6 \mathrm{~Gy})$ to the entire neuroaxis (whole brain-S2) and a local boost to the pineal region with $17 \times 1.8$ Gy up to a cumulative dose of $54.60 \mathrm{~Gy}$ on the primary tumor region. In case of the patient with the $\mathrm{II}^{\circ}$ tumor we delivered $30 \times 1.8$ Gy to the primary tumor region only. Helical tomotherapy (6 MV) was used for both patients.

Results: RT was well tolerated in both cases without grade $\geq 3$ toxicity. However, leukopenia grade 2 (2,5 G/l) occurred after craniospinal RT. The patient with the subtotal resected $\mathrm{II}^{\circ}$ tumor showed a local progression 6 months post RT, and salvage chemotherapy was initiated. The 25-year-old patient with PPTID III $^{\circ}$ is relapse-free at 29 months after RT.

Conclusion: There is no consensus for the treatment of PPTID. The molecular behaviour of PPTID resembles a PB, but clinically and prognostically the tumor behaves more like a PC. In consequence, the extent of RT remains controversial.
Distribution of metastases in PET-CT of treatment naïve lung cancer: implications for radiotherapy

Type: Clinical

Presenting Author:

Marco Radovic

Authors:

Radovic $\mathrm{M}^{1}$, Baty $\mathrm{F}^{2}$, Früh $\mathrm{M}^{3}$, Müller $\mathrm{J}^{4}$, Süveg $\mathrm{K}^{4}$, Panje $\mathrm{CM}^{1}$, Putora $\mathrm{PM}^{1}$

Institutions:

Kantonsspital St. Gallen

Department:

${ }^{1}$ Department of Radiation Oncology, ${ }^{2}$ Department of Pneumology, ${ }^{3}$ Department of Oncology, ${ }^{4}$ Department of Nuclear Medicine

Objective: The aim of this analysis was to retrospectively evaluate PET-CT findings and radiotherapy (RT) patterns in treatment-naïve stage IV lung cancer patients presented during multidisciplinary tumour board meetings.

Methods: We analyzed all PET-scans from 2012 to 2014, performed at the Kantonsspital St. Gallen in patients with lung cancer. Cranial MRI were taken into account. 157 out of 775 patients $(20 \%)$ met the criteria and were classified by histology, sites of metastases, bulky disease (defined as tumour $\geq 5 \mathrm{~cm}$ per region) and irradiated sites. Pearson's Chisquared test with Yates' continuity correction, Fisher's Exact Test, and logistic regression were applied to investigate patterns.

Results: We identified 157 patients, 25 patients with small cell lung cancer (SCLC) (16\%) and 132 patients with non-small cell lung cancer (NSCLC) (84\%).

Patients with bulky disease in hilomediastinal lymph nodes were irradiated more often in these sites (10 of 48 patients, $21 \%$ ) compared to patients with non-bulky disease $(8 / 88$ patients, $9 \%, p=0.029)$.

Brain metastases were also associated with RT (38/47 patients, $81 \%, p<0.01)$.

Metastases in the spine were also associated with RT to the spine (20/60 patients, $33 \%, p<0.01)$.

Discussion: Generally, lesions in PET-CT were not predictive for RT in most lesions despite associations in bulky hilomediastinal or brain and spinal disease. While the correlation with RT for brain metastases might decrease with the impact of the Quartz trial, potentially earlier RT to spinal lesions may prevent future complications.

Conclusion: Potentially, future analyses might demonstrate a role for prophylactic RT in spinal and bulky hilomediastinal disease in lung cancer (including NSCLC).

\section{Gain of function and analgesia from low dose radiotherapy} in musculoskeletal disorders

Type: Clinical

Presenting Author:

Rogers Susanne

Authors:

S. Rogers ${ }^{1}$, B. Eberle ${ }^{1}$, D. Vogt ${ }^{2}$, E. Meier ${ }^{1}$, L. Moser ${ }^{3}$, S. Gomez ${ }^{1}$, S. Desborough ${ }^{1}$, O. Riesterer ${ }^{1}$ I. Takacs ${ }^{4}$, P. Hasler $^{3}$, S. Bodis ${ }^{1,5}$

\section{Institutions:}

${ }^{1,3}$ Kantonsspital Aarau, ${ }^{4}$ Kantonsspital Baden, ${ }^{2}$ University Hospital of Basel, ${ }^{5}$ University Hospital Zürich

Department:

${ }^{1,4}$ Radio-Onkologie Zentrum, ${ }^{2} \mathrm{CTU},{ }^{3}$ Department of Rheumatology,

${ }^{5}$ Department of Radiation Oncology

Objectives: We prospectively evaluated changes in pain, QoL and, for the first time, functional tests after low dose radiotherapy (LDRT) in patients with the 4 commonest referrals for LDRT: lateral and medial epicondylitis (LE/ME), finger osteoarthritis (OA) and plantar fasciitis (PIF). 
Materials/methods: 157 patients over 40 yrs, with no response to at least $6 \mathrm{mths}$ of conservative therapy, were recruited. LDRT $(8 \times 0.5$ Gy, $200 \mathrm{kV}$ ) was repeated once. Handgrip dynanometers (Jamar@) and self-paced walking test were assessed by a physiotherapist. VAS and QoL questionnaires were completed at 0, 2, 6, and 12 mths.

Results: 204 sites were treated. Median changes in VAS and function were compared (12 mths vs $0 \mathrm{mths})$. $39 \mathrm{LE}$ : pain at rest $(-2.5, p<0.001)$, activity $(-6.0, p<0.001)$, handgrip (extension $+16 \mathrm{~kg}, p<0.001$, flexion $+5.2 \mathrm{~kg}, p=0.002)$, inverse correlation between pain and handgrip strength (rest $-0.6, p<0.001$, activity $-0.5, p<0.001$ ). $10 \mathrm{ME}$ : pain at rest $(-3.0, p=0.041)$, activity $(-0.4, p=0.041)$, handgrip strength $(+6.5 \mathrm{~kg}, p=0.022) .99$ finger OA: pain during activity $(-3.0, p<0.001)$, handgrip strength $(+2.5 \mathrm{~kg}, p=0.004)$, inverse correlation between pain and handgrip strength (rest $-0.41, p<0.001$, activity $-0.36, p<0.001$ ). 56 PIF: pain at rest $(-4.0, p<0.001)$, during activity $(-6.0, p<0.001)$ and improvement in walking time $(-5.0 \mathrm{~s}, p<0.001)$, correlation between pain during activity and walking time $(0.5, p<0.001)$. There was a trend towards improved QoL in patients with ME and PIF.

Conclusion: Patients with LE and ME, finger OA and PIF achieved highly significant analgesia and objective gains in musculoskeletal function which were sustained at 12 months after completion of LDRT with 4.0-8.0 Gy.

\section{Single fraction urethra-sparing prostate cancer SBRT: phase i results of the ONE SHOT trial \\ Type: Clinical}

Presenting Author:

Achard Vérane

Authors:

Verane Achard ${ }^{1}$, Ciro Franzese ${ }^{2}$, Niccolò Giaj-Levra ${ }^{5}$, Robert Förster ${ }^{4}$, Daniel Zwahlen ${ }^{3}$, Nikolaos Koustouvelis ${ }^{1}$, Aurelie Bertaut ${ }^{6}$,

Julie Blanc ${ }^{6}$, Filippo Alongi ${ }^{5}$, Matthias Guckenberger ${ }^{4}$,

Marta Scorsetti ${ }^{2}$, Raymond Miralbell ${ }^{1}$, Thomas Zilli ${ }^{1}$

\section{Institutions:}

${ }^{1}$ Hopital Universitaire Geneve, ${ }^{2}$ Milan, ${ }^{3}$ Chur, ${ }^{4}$ Zürich, ${ }^{5}$ Negrar, ${ }^{6}$ Dijon Department:

${ }^{1}$ Radio-oncology

Aims: To present the phase I results of a single fraction urethra-sparing stereotactic body radiotherapy (SBRT) for localized prostate cancer (PCa) from the single arm, multicenter phase I/II ONE SHOT trial. Methods: From 08/2017 through 12/2018, 6 patients (pts) with lowand intermediate-risk localized PCa were treated with a 19 Gy single fraction to the prostate using a VMAT technique and intrafractional motion control using intraprostatic electromagnetic transponders. The urethra received a lesser dose of 17 Gy. Genitourinary (GU) and gastrointestinal (GI) toxicity (CTCAE v4.03 grading scale), IPSS, IIEF-25 and QoL scores (EPIC) were assessed at baseline, at 5 days (5D), 6th (6 W) and 12th weeks (12 W) since SBRT. Primary endpoint of the phase I was safety as assessed by occurrence of Grade $\geq 3$ acute side effects.

Results: Median age and PSA were 75 years old and $8.1 \mathrm{ng} / \mathrm{ml}$. All pts underwent SBRT with no Grade $\geq 3$ acute side effects observed during the first 3 months. Pts experienced mostly grade 1 or $2 \mathrm{GU}$ toxicities (frequency/urgency) resolving from W6 with no grade 2 GI side effects. IPSS increased from baseline to W6 (mean, from 3.5 to 16.2) decreasing at W12 (mean, 8.8). The EPIC urinary domain mean score was 92 pretreatment, 73 at D5 and 87 at W12, while the EPIC bowel domain mean score remained stable over weeks. No impact of SBRT was observed on IIEF-25 scores and the EPIC sexual domain. The PSA values showed a bounce at D5 (median, from 8.2 to $16.5 \mathrm{ng} / \mathrm{ml}$ ) decreasing successively up to a value of $2.8 \mathrm{ng} / \mathrm{ml}$ at W12.

Conclusions: 19 Gy single-fraction urethra-sparing SBRT irradiation of the prostate was feasible and well tolerated. This is the first multicenter phase I/II trial of single dose SBRT monotherapy for localized $\mathrm{PCa}$.
Impact of vestibule dose on dizziness after radiosurgery for the treatment of vestibular schwannoma

Type: Clinical

Presenting Author:

Ekin Ermis

Authors:

E. Ermiş ${ }^{1 *}$, L. Anschuetz ${ }^{2}$, D. Leiser ${ }^{1}$, F. Wagner ${ }^{3}$, A. Raabe ${ }^{4}$, J. Abu-Isa ${ }^{4}$, M. Caversaccio ${ }^{2}$, D. Aebersold ${ }^{1}$, E. Herrmann

*equally contributed

\section{Institutions:}

Inselspital, Bern University Hospital, and University of Bern

Department:

${ }^{1}$ Department of Radiation Oncology, ${ }^{2}$ Otorhinolaryngology, Head \& Neck Surgery, ${ }^{3}$ Neuroradiology, ${ }^{4}$ Neurosurgery

Aims: To evaluate the effects of stereotactic radiosurgery (SRS) on vestibular function in patients with vestibular schwannoma (VS).

Methods: VS patients treated with SRS to 12 Gy in single fraction were reviewed. No weightings were given to vestibule during optimization in treatment planning. Pre- and post-SRS vestibular examinations (rotatory, swinging and duration of dizziness, gait instability, spontaneous nystagmus) and patient reported dizziness were assessed from patient recordings. Koos grade (I-II vs III-IV), tumor volume ( $\geq$ vs $<6.1 \mathrm{~cm}^{3}$ ), pre-SRS tinnitus-dizziness (yes vs no), target and vestibular dose (min, mean, max) and post-SRS transient volume expansion (yes vs no) were examined using linear regression analysis.

Results: Fifty-three patients treated between 2010 and 2016 were enrolled. Median follow-up was 32 months (range, 5-79). The median min., mean and max. vestibular doses were $2.6 \pm 1.6 \mathrm{~Gy}, 6.7 \pm 2.8$ $\mathrm{Gy}$, and $11 \pm 3.6 \mathrm{~Gy}$, respectively. On univariate analysis, Koos grade $(p=0.048)$, pre-SRS tumor volume (median $6.1 \mathrm{~cm}^{3}$; range, $0.8-38$ ) $(p=0.011)$, pre-SRS dizziness $(p=0.024)$ and min. vestibular dose $(p=0.033)$ were significant for patient reported dizziness. On multivariate analysis, min. vestibular dose remained significant $(p=0.029)$. In determining cutoff values, all patients who had a min. vestibular dose of $5 \mathrm{~Gy}$ and above reported dizziness.

Conclusion: There is little data available concerning vestibular toxicity related to radiotherapy and SRS. Our results reveal that $5 \mathrm{~Gy}$ and above min. vestibular doses had a significant impact on dizziness. Further investigations are needed to determine dose-volume parameters and their effects on vestibular toxicity.

\section{Volumetric-modulated arc therapy for adjuvant treatment of endometrial cancer}

Type: Clinical

Presenting Author:

Simona Cima

Authors:

S. Cima, MC Valli, A. Richetti

Institutions:

Oncology Institute of Southern Switzerland, Bellinzona-Lugano

Department:

Radiation Oncology Clinic

Aim: Volumetric modulated arc therapy (VMAT $®)$ is an advanced radiation technique that allows to achieve highly conformal dose distributions with improved target volume coverage and sparing of normal tissues compared with conventional techniques. The aim of this study was to report clinical outcomes and toxicity of endometrial cancer patients treated with adjuvant VMAT®.

Methods: From 2015 to 2018 we analyzed 27 women with invasive endometrial cancer, treated with adjuvant radiotherapy after a total abdominal hysterectomy and bilateral salpingo-oophorectomy with or without lymphadenectomy. Toxicity data were collected according 
to Common Terminology Criteria for Adverse Events v4.0. Survival curves were calculated using Kaplan-Meier method.

Results: The FIGO stages were: IB in 33\%, II in 15\%, IIIA in 11\%, IIIB in $4 \%$, IIIC in $37 \%$. The mean age was 67 years. The mean prescribed dose was $49 \mathrm{~Gy}$. Vaginal cuff brachitherapy, as a boost after EBRT, was performed in 16 patients (59\%) with a mean prescribed dose of $13 \mathrm{~Gy}$. Fourteen patients $(52 \%)$ were treated with adjuvant chemotherapy. All patients completed irradiation. No case of acute toxicity G2 or more, was observed. The 1-year and 3-year late grade $\geq 1 \mathrm{GU}$ toxicity free survival was $100 \%$ and $64 \%$, respectively. The 1-year and 3-year late grade $\geq 1$ GI toxicity free survival was $100 \%$ and $89 \%$, respectively. The 1 -year and 3 -year late grade $\geq 1$ vaginal toxicity free survival was $68 \%$ and $34 \%$. The 2-year risk for loco-regional and distant recurrence was $10 \%$ and $13 \%$, respectively. The 2 -year OS was $92 \%$.

Conclusion: Our experience showed the feasibility of this technique with good results in terms of acute and late toxicity. The short follow up and the small number of cases doesn't allow us conclusions in terms of survival outcomes.

\section{Neutrophil to lymphocyte ratio (NLR) and platelet to lymphocyte ratio (PLR) as prognostic markers in locally advanced squamous cell oropharyngeal cancer: a retrospective, observational, multicenter experience Type: Clinical}

\section{Presenting Author:}

Francesco Martucci

Authors:

Martucci $\mathrm{F}^{1}$, Cima $\mathrm{S}^{1}$, Antognoni $\mathrm{P}^{2}$, Richetti $\mathrm{A}^{1}$

\section{Institutions:}

${ }^{1}$ Oncology Institute of Southern Switzerland, Bellinzona-Lugano,

${ }^{2}$ University Hospital of Varese

Department:

${ }^{1}$ Radiation Oncology, ${ }^{2}$ Radiation Oncology

Background: Inflammatory biomarkers have been shown to be valuable prognostic markers for a variety of pathological conditions. An elevated neutrophil-to-lymphocyte ratio (NLR) and platelet-to-lymphocyte ratio (PLR) have been associated with poor prognosis in many types of cancer. The aim of this retrospective study was to evaluate the predictive value of NLR and PLR for DFS and OS in locally advanced squamous cell oropharyngeal cancer (OPSCC) patients treated with curative radiochemotherapy (CRCT).

Methods: Ninety-two patients with stage III or IV OPSCC who underwent CRCT beetween 2009 and 2016 were retrospctively analyzed. All patients were treated at University Hospital of Varese or at Oncology Institute of Southern Switzerland with Intensity-Modulated Radiation Therapy/Rapid Arc up to a dose of 66-70 Gy in 7 weeks. Concomitant to radiotherapy chemotherapy (3-weekly cisplatin, started at the first day of radiotherapy) was delivered. NLR and PLR were measured at baseline and the median value of 3 and 150, respectively, was chosen as a cut-off. Survival curves were generated using the Kaplan-Meier method. Univariate and multivariate analyses using Cox proportional hazards model were used to identify predictors of progression-free survival (PFS) and overall survival (OS).

Results: Median follow-up was 39 months. Univariate analysis showed that PLR and HPV status were significant predictors of DFS $(\mathrm{HR}=2.3$, 95\% CI $1.0-5.1 ; P=0.04),(\mathrm{HR}=4.8,95 \%$ CI $1.4-16.6 ; P=0.01)$. However, the HPV status remained an independent predictor of DFS in the multivariate analysis $(\mathrm{HR}=5.8,95 \%$ CI $1.4-23.9 ; P=0.02)$. The only significant predictor of OS was HPV status $(\mathrm{HR}=18.2,95 \% \mathrm{CI}$ 5.6-59.3; $P<0.0001)$.

Conclusions: A high pre-treatment PLR might be a valuable predicative biomarker of poor prognosis for patients with OPSCC. Further studies are needed to investigate the prognostic value of inflammatory markers.
Automation in radiation therapy: Effects and challenges for RTT work

Type: Clinical

Presenting Author:

Laurent Marmy

Author:

L. Marmy ${ }^{1,2}$, V.N. Ho ${ }^{1}$

Institutions:

${ }^{1} \mathrm{HFR}-$ Fribourg Hospital, ${ }^{2} \mathrm{HESAV}$, Lausanne

Department:

${ }^{1}$ Radiation Oncology Department, ${ }^{2}$ filière TRM

Aims: Automation in radiation therapy involves adaptation of RTTs' work. Our qualitative study aims analyze this phenomenon, particularly its effects and challenges for the profession and the multi-disciplinary team.

Method: We conducted eight semi-structured interviews in western Switzerland. The data was analyzed using traditional a content analysis method. We bundled our results in 5 main topics: automation in RTTs' work, speeches automation, automated environment challenges caused by automation in the profession and evolution of the profession. Results: Automation has effects on clinical practice and more particularly on repetitive procedures, quality and safety, teamwork and patient care. An important point to note is the lack of involvement of the RTT in the implementation processes of automated devices. The economic issue is considered as the main decision-making factor of hierarchy while professional, technological and relational issues are often underestimated.

Conclusion: Automation has a significant impact on RTT activity. According to some of them, the profession should evolve to adapt to its new work environment. Professionals should also strengthen their professional identity in order to obtain role recognition in the context of automated radiation-oncology.

\section{Multimodal treatment for locally advanced oral cavity cancer:} mono-institutional experience

Type: Clinical

Presenting Author:

Francesco Martucci

Authors:

Martucci F, Cima S, Borgonovo G, Richetti A.

Institutions:

Oncology Institute of Southern Switzerland, Bellinzona-Lugano Department:

Radiation Oncology Clinic

Objectives: Oral cavity squamous cell carcinoma (SCCOC) represents a common entity of all head and neck malignant tumors. Presentation with advanced disease is common. The standard of care is primary surgical resection with or without postoperative adjuvant therapy. Because of the functional and cosmetic relevance of the oral cavity, different conservative approaches, as exclusive radiotherapy or concomitant chemo-radiation have been used. The aim of this report is to check the outcome of patients (pts) with SCCOC treated in our radiation oncology clinic in the last 10 years

Materials and methods: Fifty-four pts with SCCOC were treated in our clinic from January 2008 to December 2017. 19 pts (35\%) were stage III and 35 pts were stage IVA (65\%), 44 pts $(82 \%)$ were staged with PET/CT at diagnosis. 34 pts $(63 \%)$ were treated with surgery, half of them resulted with a positive margin (R1-2). All pts were treated with radiotherapy (exclusive or postoperative) with Volumetric Arc Therapy/RapidArc ® up to a curative dose, 29 pts (54\%) received concomitant chemotherapy platinum based. Overall survival (OS) was defined as the time from diagnosis to death from any cause. Progres- 
sion-free survival (PFS) was defined as the time from radiotherapy to the first loco regional recurrence and/or metastases. Survival curves were calculated using Kaplan-Meier method.

Results: The median age was 66 (range 34-90) years. The mean follow up was 50 (13-135) months. The 3-years and 5-years PFS was $45 \%$ and $10 \%$, respectively. Twenty-nine pts $(54 \%)$ recurred to a loco-regional site, 4 pts $(7 \%)$ developed metastatic disease. The 3-years and 5 -years OS was $58 \%$ and $33 \%$, respectively; for 5 pts (9\%) the death was not cancer related.

Conclusions: SCCOC remains a poor prognosis site within head and neck cancers. Improvements have been done, with multimodal treatments, in terms of outcome results. However complete and radical surgical resection is considered, today, the standard of care due to the advanced surgical techniques, mostly for early stages of disease.

\section{Could be SIB the (right) answer? A case report}

Type: Clinical

\section{Presenting Author:}

Vite Cristina

Authors:

Vite C., Ballerini G., Corradini N., Negretti L., Urso P., Franzetti-Pellanda A.

Radiotherapy, Clinica Luganese Moncucco

Institutions:

Clinica Luganese Moncucco

Department:

Radiotherapy

Background: 63-year-old patient diagnosed with invasive ductal carcinoma of the right breast, upper internal quadrant, T2 N1 M0, G3 staging received a quadrantectomy, as well as breast reduction of the contralateral breast.

In 1990 the patient was already diagnosed with invasive ductal carcinoma of the left breast (T2 N1 M0, G2) and received a quadrantectomy and axillary lymphnode dissection followed by external beam radiotherapy (total dose $55 \mathrm{~Gy}$ ).

Aim: Finding the best radiotherapeutic approach to obtain optimal coverage of the planning target volumes (PTVs) together with sparing of the contralateral breast (CB), given to the patient's clinical history. Methods: The following irradiation techniques, optimized on the Tomotherapy ${ }^{\circledR}$ system, and the following radiotherapy schedules were considered:

50 Gy: (2 Gy/fraction) on the whole breast (WB) with TomoDirect ${ }^{\circledR}$ technique (TD), with a sequential boost on the tumor bed (TB) of $10 \mathrm{~Gy}\left(2 \mathrm{~Gy} /\right.$ fraction) with TomoHelical ${ }^{\circledR}$ technique (TH).

$50 \mathrm{~Gy}$ : (2 Gy/fraction) on the WB with TH, with a sequential boost on the TB of $10 \mathrm{~Gy}(2 \mathrm{~Gy} /$ fraction) with TD.

$50 \mathrm{~Gy}:(2 \mathrm{~Gy} / \mathrm{fraction})$ on the WB with an integrated simultaneous boost (SIB) on the TB of $58.75 \mathrm{~Gy},(2.35 \mathrm{~Gy} /$ fraction) with TH.

The comparison was based on the coverage of the PTV and on the doses of the CB and the ipsilateral lung (IL).

Results: Due to the patient's anatomical configuration, sparing the contralateral breast makes the TD technique inappropriate (coverage WB $89.7 \%$, TB 72.8\%; CB D2\% 9.2 Gy).

The coverage with $\mathrm{TH}$ technique was acceptable for the WB (94.2\%), but unacceptable for the TB (90.4\%).

The SIB approach guaranteed a good coverage for both PTVs (WB $96.9 \%$, TB 96.1\%), with a minimum variation of the organ's doses (IL V20Gy $9 \%$ vs 8\%; CB D2\% 7.9 Gy vs 6.6 Gy).

Conclusion: SIB was considered the most appropriate approach since it guaranteed a good target coverage, in particular for the tumor bed, together with sparing the organs at risk.

Promoting a SIB approach for all patients is outside the scope of the present work, but authors believe it should be individually considered.
Impact of prophylactic cranial irradiation on 18F-FDG cerebral metabolism in small cell lung cancer patients

Type: Clinical

Presenting Author:

Shaïma El Chammah

Authors:

S. El Chammah ${ }^{2}$, G. Allenbach ${ }^{1}$, R. Jumeau ${ }^{2}$, J. O. Prior ${ }^{1}$, M. Nicod Lalonde ${ }^{1}$, P. Genoud ${ }^{1}$, N. Schaefer ${ }^{1}$, M. Meyer ${ }^{1}$

Institutions:

CHUV, Lausanne

Department:

${ }^{1}$ Nuclear Medicine and Molecular Imaging Department, ${ }^{2}$ RadioOncology Department

Aims: In limited-disease small cell lung cancer (SCLC) patients, prophylactic cranial irradiation (PCI) is associated with increased survival and with cognitive impairment. Although the exact mechanisms underlying this cognitive impairment remain poorly understood, hippocampal sparing (HP) during PCI has shown promise in preventing cognitive impairment. Our study aims to evaluate the short-term impact of PCI on ${ }^{18} \mathrm{~F}$-FDG brain metabolism.

Methods: We retrospectively included 18 SCLC patients. All patients received an intensity-modulated radiotherapy with a prescribed dose of $25 \mathrm{~Gy}$ in 10 fractions, except for one patient (26 Gy in 13 fractions). Among these patients, 10 beneficiated from HP PCI. ${ }^{18} \mathrm{~F}-\mathrm{FDG}$ PET/CT was performed on average 146 days before and 294 days after PCI. An automatic anatomical brain segmentation of the ${ }^{18} \mathrm{~F}-\mathrm{FDG}$ PET was performed using Combined-AAL Atlas from MI-Neurology Software (Syngo.Via, Siemens). For all the regions, we calculated SUVratio using brainstem as reference region $(\mathrm{SUVr}=\mathrm{SUVmean} / \mathrm{Brainstem} \mathrm{SU}$ Vmean) and we compared SUVr before and after PCI using a Wilcoxon test with a level of significance of $p \leq 0.05$.

Results: We showed a significant decrease in ${ }^{18} \mathrm{~F}-\mathrm{FDG}$ brain metabolism after PCI in most anatomical regions: basal ganglia (1.39 vs 1.33 , $p=0.018)$, central regions ( 1.32 vs $1.26, p=0.012)$, cingulate cortex (1.32 vs $1.23, p=0.003)$, corpus striata ( 1.42 vs $1.35, p=0.016)$, frontal cortex (1.31 vs $1.24, p=0.012)$, occipital cortex ( 1.48 vs $1.41, p=0.02)$, precuneus (1.46 vs $1.40, p=0.028)$ and lateral temporal cortex (1.33 vs $1.27, p=0.012$ ). Results also show a clear tendency for decrease in the parietal cortex $(1.33$ vs $1.29, p=0.053)$. No significant difference was found in the cerebellum $(1.24$ vs $1.21, p=0.85)$ and mesial temporal cortex including the hippocampus ( 1.03 vs $1.00, p=0.16$ ).

Conclusion: PCI leads to short-term decrease of ${ }^{18} \mathrm{~F}$-FDG brain metabolism, except in cerebellum and in regions that have been spared during PCI.

\section{First report of radical radiotherapy for anorectal basal cell carcinoma \\ Type: Clinical}

Presenting Author:

Stephanie Brendel

Authors:

Brendel $^{1}$, Rogers ${ }^{1}$, Gomez $^{1}$, Memeti $^{2}$, Bodis ${ }^{1}$

Institutions:

Kantonsspital Aarau, ${ }^{2}$ Spital Zofingen

Department:

${ }^{1}$ Radiation Oncology, ${ }^{2}$ Surgery

Aims: Only $1 \%$ of basal cell carcinomas (BCC) are perianal and $0.2 \%$ of anorectal tumors are BCCs [1]. Treatment is usually surgical, with or without adjuvant radiotherapy (RT), but immunotherapy and chemotherapy have also been described [4]. We present the first case report of a patient with a large anorectal BCC treated with sphincter-preserving definitive RT. 
Methods: A fit, 68 year old male presented with a $3 \mathrm{mth}$ history of discomfort on sitting, without micturition or defecation-related symptoms. He had had multiple BCCs in sun-exposed areas but had no other risk factors. A $4 \times 2 \times 3 \mathrm{~cm}$ ulcer was visible at 3-6 o'clock extending up to the linea dentata. Biopsy showed a $90 \%$ solid/10\% sclerodermiform BCC, p16 negative, Ber-EP4, typical of BCC [1], was weakly positive. Staging confirmed perianal infiltration without locoregional or distant metastatic spread. The tumorboard recommendation was definitive local RT with the option of salvage rectum amputation. $60 \mathrm{~Gy}$ in 30 daily fractions was delivered to the primary tumor without elective nodal RT using VMAT.

Results/discussion: BCCs are rarely anorectal and a conclusive biopsy is essential to make the diagnosis. It is important to distinguish BCC from squamous (particularly basaloid) cell anal carcinoma (SCC). 30$50 \%$ of patients with basaloid SCC present with metastases [2], which are extremely rare in BCC $(<1: 1000)$ [4]. Perianal BCCs typically occur in males, average age at diagnosis is $67-73$ years $[3,5]$. Other than UV light, risk factors include immune deficiency, arsenic exposition, sexually transmitted diseases and chronic dermatitis. The patient developed no acute toxicities other than radiodermatitis (CTCAE III). MRI 3 mths after RT showed a good partial response and clinically sphincter function remains normal.

Conclusion: Conclusive histopathology is mandatory to provide personalized treatment for perianal BCCs. In large anorectal cases, we recommend sphincter-preserving primary RT. Regular follow up for local control is planned.

\section{Experience and outcomes of radiosurgery with a single isocentre for 2-10 brain metastases \\ Type: Clinical}

\section{Presenting Author:}

Susanne Rogers

Authors:

Rogers $\mathrm{S}^{1}$, Lomax $\mathrm{N}^{1}$, Alonso $\mathrm{S}^{1}$, Eberle $\mathrm{B}^{1}$, Gomez Ordonez $\mathrm{S}^{1}$, Schürkens $\mathrm{J}^{1}$, Rabe $\mathrm{E}^{1}$, Fandino $\mathrm{J}^{2}$, Riesterer $\mathrm{O}^{1}$, Lutters $\mathrm{G}^{1}$, Bodis $\mathrm{S}^{1}$

Institutions:

${ }^{1,2}$ Kantonsspital Aarau, ${ }^{1}$ Kantonsspital Baden

Department:

${ }^{1}$ Radioonkologie, ${ }^{2}$ Neurochirurgie

Purpose: Radiosurgery (RS) planning and delivery for multiple brain metastases (BM) can be facilitated using a single isocentre. We have reviewed the feasibility and efficacy of the single isocentre dynamic conformal arc (SIDCA) technique.

Methods: 51 patients $(2-10 \mathrm{BMs}$ ) have been treated on a Novalis STx Linac. 20 consecutive patients, planned with SIDCA (Elements Multiple Metastases v1.5, Brainlab), were analysed. Dose prescription was $1 \times 20 \mathrm{~Gy},(\mathrm{~V} 20 \mathrm{~Gy}=99 \%$, with Dmax 115-130\%), with a $1 \mathrm{~mm}$ margin. Results: 20 patients had a total of 72 BMs. $70 \%$ had NSCLC and the median age was $70 \mathrm{yrs}$ (51-81 yrs). In 8/20 patients, BMs were grouped into 2-3 isocentres for clinical indications (e.g. fractionated RS due to volume or postop) or rotational uncertainties ( $>4 \mathrm{~cm}$ from isocentre) rather than increase the margin. Dose prescriptions were 52/72 BMs: $1 \times 20$ Gy, 7/72: $1 \times 15-18$ Gy, $13 / 72: 5 \times 5-6$ Gy according to patient and tumor factors. For single fractions (59 BMs), mean dose was 22.1 Gy $(15.8-23.95 \mathrm{~Gy})$ and median volume was $0.29 \mathrm{~cm}^{3}\left(0.09-2.78 \mathrm{~cm}^{3}\right)$ with a median cumulative PTV volume of $1.08 \mathrm{~cm}^{3}\left(0.36-7.74 \mathrm{~cm}^{3}\right)$ per patient. At 6 week follow-up, 3 patients required steroids. 5 patients developed distant BMs 3-6 mths after RS, of whom 3 received further SIDCA RS. 11 patients have died (median OS $6 \mathrm{mths}, 2-13 \mathrm{mths}$ ); 9/11 patients $(82 \%)$ with control of the irradiated BMs (3 CR, 3 PR, 4 SD) at last follow-up.

Conclusions: SIDCA has been successfully implemented for patients with multiple BMs and is in routine use for patients with single BMs as it decreases the duration of planning, verification and reporting. The irradiation of multiple BMs up to $4 \mathrm{~cm}$ from the isocentre with the same fractionation scheme and a $1 \mathrm{~mm}$ margin is feasible, efficient and effective in daily practice. Local control rates $>80 \%$ are equivalent to those achieved with RS for single BMs.

Salvage radiotherapy for pelvic nodal recurrences of prostate cancer after radical prostatectomy: A national survey on patterns of practice

Type: Clinical

Presenting Author:

Dr Panje or Dr Putora

Authors:

C. Panje ${ }^{1}$, T. Zilli², A. Dal Pra ${ }^{3}$, W. Arnold ${ }^{4}$, K. Brouwer ${ }^{5}$, H. Garcia

Schüler $^{6}$, S. Gomez ${ }^{7}$, F. Herrera ${ }^{8}$, K. Khanfir ${ }^{9}$, A. Papachristofilou ${ }^{10}$, G. Pesce ${ }^{11}$, C. Reuter ${ }^{12}$, H. Vees ${ }^{13}$, D. Zwahlen ${ }^{14}$, P. M. Putora ${ }^{1}$

\section{Institutions:}

${ }^{1}$ Kantonsspital St. Gallen, ${ }^{2}$ Hôpitaux Universitaires de Genève, ${ }^{3}$ Inselspital Bern, ${ }^{4}$ Luzerner Kantonsspital, ${ }^{5}$ Stadtspital Triemli, Zürich, ${ }^{6}$ Universitätsspital Zürich, ${ }^{7}$ Kantonsspital Aarau, ${ }^{8}$ Centre Hospitalier Universitaire Vaudois, Lausanne, ${ }^{9} \mathrm{Hôpital} \mathrm{du} \mathrm{Valais,}$ Sion, ${ }^{10}$ Universitätsspital Basel, ${ }^{11} \mathrm{EOC}$ Bellinzona, ${ }^{12}$ Kantonsspital Münsterlingen, ${ }^{13}$ Klinik Hirslanden, Zürich, ${ }^{14}$ Kantonsspital

Graubünden, Chur

Department:

${ }^{1}$ Departments of radiation oncology

Aim: There is currently no standard for local salvage therapy of intrapelvic nodal oligorecurrences of prostate cancer (PCA) after radical prostatectomy. Elective nodal radiotherapy (ENRT) or stereotactic body RT (SBRT) are both valid treatment strategies. The aim of our study was to assess current practice in Switzerland.

Method: Treatment recommendations of 14 participating Swiss radiation oncology centers were collected and converted into decision trees in an iterative process using the objective consensus methodology and analyzed for consensus und differences.

Results: For unfit patients, androgen-deprivation therapy (ADT) alone was recommended by more than $70 \%$. For fit patients with unfavorable tumor characteristics, the majority of the centers recommended ENRT + ADT for up to five nodal recurrences. In fit patients with favorable tumor characteristics, there were low levels of consensus and a wide variety of recommendations, especially for one and for four nodal recurrences. Focal SBRT was offered by $64 \%$ of the centers, most commonly as a 5 -fraction course. PSMA PET/CT was recommended by $93 \%$ of the centers for restaging.

Conclusion: Metastasis-directed therapy for pelvic oligorecurrences of PCA is commonly proposed in selected patients in Switzerland, with considerable treatment variations between centers. The best treatment strategy is not yet defined and it is explored in ongoing studies such as the STORM trial (NCT03569241).

Evaluation of an automatic planning solution for breast cancer Type: Clinical

Presenting Author:

Jessica van Rossum

Authors:

J. van Rossum, N. Andratschke, M. Guckenberger, C. Linsenmeier, S. Tanadini-Lang, L. Wilke

Institutions:

Universitätsspital Zürich

Department:

Klinik für Radio-Onkologie

Aims: Radiotherapy is a standard treatment for breast cancer. Techniques and programs are advancing to make these planning techniques 
more efficient and less user dependent, while achieving the same or better dosimetric outcomes. This planning study was done to investigate whether the automatic planning solution EZ fluence (RADformation) is more efficient in generating $3 \mathrm{D}$ conformal irregular surface compensator (ISC) plans than manually creating the 3D conformal ISC plans in the Eclipse planning system (Varian, V15.6).

Methods: Fourteen breast patients were randomly selected with a range of left (9) and right-side (5) breast PTVs. One experienced dosimetrist created a 3D conformal irregular surface compensator (ISC) plans using the standard method of painting the fluence and expanding the dose beyond the skin. The same dosimetrist repeated the plan-creation using the automatic solution. For the PTV, coverage (D95\%), $\mathrm{D} 2 \%$ and $\mathrm{D}_{\max }$ dose were collected. For the lung, $\mathrm{D}_{\text {mean }}$ and V5\% were collected and for the heart $\mathrm{D}_{\text {mean }}, \mathrm{D} 2 \%$ and $\mathrm{D}_{\max }$. The total planning time was recorded for each of the two systems.

Results: The median coverage of the PTV evaluated by the D95\% was $94.1 \%$ (88.7-95.8\%) for the manual plans compared to $94.8 \%$ $(90-96.9 \%)$ for the automatic plans $(p=0.08)$. The maximum dose was slightly higher for the manual plans (median 105.8\%, range $104.7-105.9 \%$ ) compared to $104.5 \%$ median (range 103.2-105.9\%) $(p=0.003)$. No statistical difference was found for the dose to the heart and lungs. The median planning time was significantly reduced from $44(20-70)$ to $25(14-44)$ minutes with the use of the automatic planning $(p=0.001)$.

Conclusions: Automatic treatment planning significantly reduced the treatment planning time by nearly a factor of two, while the treatment plan quality was equally high to our manual planning.

\section{Electromagnetic localization and real-time tracking for prostate cancer radiotherapy: impact of metal hip prostheses Type: Clinical}

Presenting Author:

Marta Bottero

Authors:

Bottero M, Dipasquale G, Jaccard M, Zilli T

\section{Institutions:}

Geneva Univeristy Hospital

Department:

Radiation oncology

Purpose/objective: Metal hip prostheses represent a former contraindication to electromagnetic transponders (EMT) for prostate cancer (PCa) radiotherapy (RT) guidance. Aim of this study was to assess the EMT ability and accuracy to track and localize prostate movements in PCa patients with metallic hip prostheses (MHP) treated with curative $\mathrm{RT}$.

Materials/Methods: Datasets of 7 PCa patients with MHP (3 with bilateral and 4 with unilateral MHP) treated with curative RT between 2016 and 2018 were analyzed. All patients were implanted with $3 \mathrm{Ca}-$ lypso ${ }^{\circledR}$ beacons EMT (Varian Medical Systems, Palo Alto, CA) and the median distance between EMT and the nearest MHP was $4.96 \mathrm{~cm}$ (range, 4.18-6.68). Different RT schedules were used, with a median delivered dose of $60 \mathrm{~Gy}$ (range, 36.25-74 Gy) in 15 fractions (range, 5-37). Localization and tracking accuracy was quantified for each treatment fraction by the geometric residual value calculated as the difference between the planned and the measured transponders position. Results: The Calypso system was able to localize and track the prostate position without any interference due to MHP in all treatment fractions $(n=90)$. Treatments were interrupted when the EMTs barycenter was out of the tolerance. In one patient with bilateral MHP, the Calypso system was able to detect during the 1st fraction the migration of one EMT, as confirmed by a new planning CT scan. The median geometric residual value was $0.07 \mathrm{~cm}$ (range, $0.03-0.22 \mathrm{~cm}$ ) and in only one of the 90 treatment fractions the vector displacement was above the limit of $0.2 \mathrm{~cm}$.
Conclusions: In PCa patients, intrafractional motion control with intraprostatic EMT is not affected by the presence of unilateral or bilateral MHP. With a negligible average tracking error, this is the first clinical experience demonstrating the tracking and localization ability of the Calypso system during curative RT in PCa patients with MHP.

\section{5 years of intracranial fractionated radiosurgery treatments} with a non-dedicated accelerator: our experience

Type: Clinical

Presenting Author:

Vite Cristina

Authors:

Vite C, Negretti L, Corradini N, Urso P, Ballerini G, Franzetti-

Pellanda A.

Institutions:

Clinica Luganese Moncucco

Department:

Radiotherapy Department

Aim: Retrospective analysis of our 5-year experience in intracranial fractionated radiosurgery with a non-dedicated linear accelerator.

Methods: Data of intracranial radiosurgery treatments from 2014 to 2019 were collected. Primary clinical disease, age, dose prescription and survival time after radiotherapy, if available, were registered.

Treatment plans were optimized and delivered with the Tomotherapy ${ }^{\circledR}$ HDA system (v.5.1.2) from Accuray ${ }^{\circledR}$. Patient utilized a personalized immobilization system, comprised of an individual head support and a 5-pt thermoplastic mask.

Treatment plans were analyzed in terms of planning target volume (PTV): equivalent diameter $\left(\mathrm{D}_{\text {equiv }}\right)$, near-minimum dose (D98\%), conformity and gradient index (CI, GI), brain volume receiving 12 Gy (V12Gy).

A MVCT scan was performed before each treatment fraction and registration shifts were analyzed as random and systematic errors.

Results: We treated 24 patients with a median age of 64 years [49-82 yrs] and a total of 30 PTVs of which 3 cases had multiple targets, 1 case had two dose levels on the surgical bed with a SIB to residual disease (median volume $=4.7 \mathrm{~cm}^{3}\left[0.8-18.0 \mathrm{~cm}^{3}\right]$, median $D_{\text {equiv }}=20.8 \mathrm{~mm}$ [11.5-32.5 mm]).

Median prescription dose was 33 Gy [25-36 Gy], with a schedule of 5 fractions (67\%), 3 fractions (23\%), 6 fractions (10\%).

Concerning dosimetry, the median D98\% was $99.5 \%$ [90.5$104.4 \%$ ], with $\mathrm{CI}=1.2$ [1.1-1.7], $\mathrm{GI}=4.6$ [3.4-7.3] and a median brain $\mathrm{V} 12 \mathrm{~Gy}=29.3 \mathrm{~cm}^{3}\left[9.5-111.2 \mathrm{~cm}^{3}\right]$.

Setup registrations gave a systematic and random errors of $1.0 \mathrm{~mm}$ and $1.1 \mathrm{~mm}$ in lateral direction, $0.7 \mathrm{~mm}$ and $1.1 \mathrm{~mm}$ in vertical and longitudinal, respectively.

Primary diseases were from lung (50\%), breast (17\%) and esophagus $(8 \%)$. Median survival time after treatment was 9.1 months [0.8$44.3 \mathrm{mos}$ ]; survival rate at 12 months was $58 \%$.

On the whole, our results are consistent with scientific literature. Conclusion: Our experience in intracranial fractionated radiosurgery, even if with a non-dedicated linear accelerator, gave appreciable results, that will be also validated by an international external audit.

Percussion assisted radiation therapy in Hodgkin lymphoma and breast cancer allows a marked reduction in heart dose Type: Clinical

\section{Presenting Author:}

André Durham

Institutions:

CHUV

Department:

Radiation-Oncology 


\section{Authors:}

André-Dante Durham ${ }^{1}$, Wendy Jeanneret Sozzi ${ }^{1}$, Raphaël Jumeau ${ }^{1}$, Alban Lovis ${ }^{2}$, Julien Simons 3 , Olivier Long ${ }^{3}$, Flore Buela ${ }^{3}$, Adam Ogna ${ }^{2}$, Anne Cairoli ${ }^{4}$, Susana Rocha Do Carmo Leal ${ }^{1}$, Michele Zeverino ${ }^{5}$, David Patin ${ }^{5}$, Véronique Vallet ${ }^{5}$, Raphaël Moeckli ${ }^{5}$, Jean Bourhis ${ }^{1 *}$, Mahmut Ozsahin ${ }^{1 *}$

\section{Institutions:}

${ }^{1}$ CHUV, ${ }^{5}$ Institute of Radiation Physics, Lausanne

Departments:

${ }^{1}$ Radiation Oncology, ${ }^{2}$ Pulmonology, ${ }^{3}$ Physiotherapy, ${ }^{4}$ Hematology

*Equally contributed

Aims: Deep inspiration breath hold is commonly used in the treatment of left-sided breast cancer and mediastinal Hodgkin lymphoma patients to reduce the dose to the heart. We developed a high frequency non-invasive ventilation (HF-NIV) system that is able to suppress respiratory motion for periods of up to $20 \mathrm{~min}$ in a very deep inspiratory state and report the results of the first series of patients treated by this percussion assisted radiation therapy (PART).

Methods: Patients treated for mediastinal Hodgkin lymphoma and left sided locoregional breast cancer were offered treatment with our novel system and planning CT scans in standard and HF-NIV conditions were obtained. Treatment plans were calculated in these two settings and compared. Treatments could be performed by VMAT or Helical Tomotherapy.

Results: PART allowed a significant reduction in the average heart dose in all the patients, and we therefore treated the first 16 patients, 8 Hodgkin and 8 breast cancer, with this HF-NIV system successfully.

Conclusions: PART can be used routinely to reduce the dose to the heart.

\section{Prospective study on the impact of adjuvant radiotherapy on the biological and clinical parameters of patients with right- sided breast cancer}

Type: Clinical

\section{Presenting Author:}

David C. Lauffer

Authors:

D. C. Lauffer, P.A. Kuhn, S. U. Thalmann, T.F. Breuneval, M. Laouiti, P. Miglierini, P.-A. Tercier, G. Risse, A. S. Allal

\section{Institutions:}

Fribourg Hospital

Department:

Radiation Oncology Department

Aims: During right-sided breast cancer radiotherapy (RT), the liver may be partially irradiated. Information on the dose delivered to the liver and its potential impact is lacking. Thus, we performed a prospective study to evaluate the dose to the liver, the biological parameters and the acute toxicities during RT. The study was approved by the Ethics Committee Vaud.

Methods: From June 2016 to May 2017, 34 women with right-sided breast cancer, treated with adjuvant RT in one institution (Fribourg), were enrolled. The RT schedule was either hypo-fractionated or standard (42.56-50 Gy) +/- a boost. Patients had blood and liver enzyme counts before RT and during the last week of RT. The acute toxicity was evaluated using the CTCAE v4.0 criteria. Parametric and non-parametric tests were used to compare the mean values and the potential correlations between the different variables.

Results: During RT there was a significant decrease in the white blood cells and thrombocyte counts. Some hepatic parameters changed over time but the mean differences were not significant. However, a significant correlation was observed between certain hepatic test alterations (ASAT, ALAT, Alkaline phosphatase) and the volume of the irradiated liver and/or the mean delivered dose. The liver mean dose was signif- icantly correlated to the volume of the right lung $(p=0.008)$. On first analysis, no correlation between the clinical toxicities and the biological test alterations was found. Detailed data will be presented. Conclusion: With standard RT techniques, unnecessary liver irradiation was documented and significant hepatic test alterations observed. The correlation of the liver mean dose with the right lung volume suggests that deep inspiration breath hold technique may represent a way to decrease the liver dose. The clinical impact of the liver test alterations needs to be evaluated in further, larger studies.

\section{Automatic vs manual beam interruption in ocular proton therapy: a retrospective study}

Type: Clinical

\section{Presenting Author:}

Riccardo Via

Authors:

R. Via ${ }^{1}$, F. Hennings ${ }^{1}$, G. Fattori ${ }^{1}$, A. Pica ${ }^{1}$, A. J. Lomax ${ }^{1}$, D. C. Weber ${ }^{1}$, G. Baroni ${ }^{2}$, J. Hrbacek ${ }^{1}$

\section{Institutions:}

${ }^{1}$ Paul Scherrer Institut, Villigen, ${ }^{2}$ Politecnico di Milano Department:

${ }^{1}$ Center for Proton Therapy, ${ }^{2}$ Dipartimento di Elettronica, Informazione e Bioingegneria

Introduction: An eye tracking system (ETS) for eye localization, based on stereoscopic optical imaging and multiple sources infrared illumination, monitored eye movements during 60 fractions of proton therapy treatments at Paul Scherrer Institut. The device performance as a beam gating system was retrospectively evaluated against the clinical standard based on visual inspection of eye movement and manual beam interruption.

Materials and methods: A threshold for beam gating was defined upon consideration on standard safety margin $(2.5 \mathrm{~mm})$ applied in ocular proton therapy. By factoring penumbra $(1.0 \mathrm{~mm})$, set-up errors $(0.5 \mathrm{~mm})$ and clinical uncertainties in target volume definition $(1.0 \mathrm{~mm})$, the maximum allowed target deviations resulted in $0.71 \mathrm{~mm}$.

The threshold was retrospectively applied to identify True Positive (TP) and False Positive (FP) as manual interrupt triggered when target-beam misalignment was over and below threshold, respectively (with associated delays). Also, an over-threshold target deviation lasting at least $0.5 \mathrm{~s}$ and not concomitant with manual interrupt was labelled as a False Negative (FN).

Results: Average target motion varied between fractions where interrupts occurred ( 9 fractions, $0.44 \pm 0.33 \mathrm{~mm}$ ) or were not triggered (51 fractions, $0.35 \pm 0.26 \mathrm{~mm}$ ). Amongst the seventeen triggered manual interruption, eight were identified as TP and nine as FP. The mean delay associated with TP was $220 \mathrm{~ms}$. Also, 11 visually undetected above-threshold eye movements (FN) were found in the dataset. On average, the duration of FN instances was $0.64 \mathrm{~s}$.

Conclusion: The clinical standard for motion monitoring in OPT is subject to relevant uncertainties and intra-user variability. The results show the benefit of using an ETS for gated irradiation. An automatic, quantitative and reliable measurement of target deviation will avoid unnecessary beam interruption and guarantee a swifter treatment course. 
Hypofractionated volumetric modulated arc therapy (VMAT) in the treatment of advanced oesophageal cancer

Type: Clinical

Presenting Author:

Letizia Deantonio

Authors:

L. Deantonio, S. Cima, A. Richetti, MC Valli

\section{Institutions:}

Istituto Oncologico della Svizzera italiana, Bellinzona

Department:

Radiotherapy

Aim: To evaluate feasibility, safety, toxicity profile, and dosimetric results of VMAT to deliver hypofractionated radiotherapy in locally advanced oesophageal cancer patients, unfit for a multimodality strategy. Methods: From 2010 to 2017, twenty-two patients were treated with hypofractionated VMAT for exclusive setting. Dose prescription of 40 Gy in 16 fractions (EQD2 41.7 Gy considering an alfa/beta of 10, and 44 Gy considering an alfa/beta of 3 ) depended on a clinical scenario of patients unfit for a standard neoadjuvant/definitive treatment.

Results: Eight patients were in stage IVB (36\%), and the other 14 $(64 \%)$ were in stage II-III. Eight patients $(36 \%)$ presented a grade 3 baseline dysphagia. RT was generally well tolerated, no patient interrupted the daily treatment. Acute toxicity was generally mild. According to the RTOG/EORTC toxicity scale, G1 toxicities were observed for weight loss (32\%), fatigue (59\%), and nausea/vomiting (18\%). During treatment, $86 \%$ of patients experienced a stable or an improvement of baseline dysphagia, while only 3 patients reported a worsening of esophagitis. Among the 19 patients suitable for short term follow-up, 15 (79\%) had a complete clinical recovery (G0-1) of the symptomatic moderate/severe dysphagia. Mean overall survival was 8.7 months (range 1-43 months).

Two coplanar arcs were employed for VMAT delivery. Dosimetric results were consistent in term of both target coverage and normal tissue sparing. For setup verification, kilovoltage cone-beam CT image-guidance was employed for all patients.

Conclusion: Hypofractionated VMAT was feasible, safe, and effective to deliver definitive radiation in locally advanced oesophageal cancer patients, non-suitable for a standard treatment.

\section{Leptomeningeal disease following stereotactic radiotherapy for resected brain metastases \\ Type: Clinical}

Presenting Author:

Susanne Rogers

Authors:

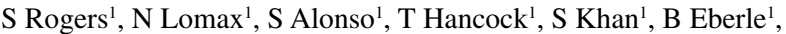
S Gomez ${ }^{1}$, O Riesterer ${ }^{1}$, J Fandino ${ }^{2}$, S Bodis ${ }^{1}$

\section{Institutions:}

${ }^{1,2}$ Kantonsspital Aarau, Kantonsspital Baden

Department:

${ }^{1}$ Radiation Oncology Center, ${ }^{2}$ Neurosurgery

Introduction: Leptomeningeal disease (LMD) can occur after brain metastasectomy due to dissemination of tumour cells into the cerebrospinal fluid. Postoperative whole brain radiotherapy (WBRT) reduces the incidence but neurocognitive impairment and lack of survival benefit underlie the preference for postop radiosurgery (SRS) or hypofractionated stereotactic radiotherapy (hfSRT). We evaluated clinical outcomes, particularly LMD, following hfSRT to the surgical cavity. Materials and methods: 45 consecutive patients (48 cavities) were evaluated for LMD, local control (LC), overall survival (OS) and radionecrosis (RN). Prior to 2015, the prescription was homogeneous with a $5 \mathrm{~mm}$ margin (10 patients) and is now D99\%PTV, Dmax 125\% with a
$2 \mathrm{~mm}$ margin and $5 \mathrm{~mm}$ dural extension. Median dose was $5 \times 6$ Gy according to the constraint V20Gy $\mathrm{y}_{\text {(brain-CTV) }} \leq 20 \mathrm{c}^{\mathrm{m} 3}$. Follow-up MRI was performed every 3 months.

Results: 24/44 patients are alive, of whom 7 were treated prior to 2015 [med. OS 52 mths (29-83 mths)]. Median OS of all patients was 15 months (3-83 mths) and 1 patient was lost to follow-up. 12 month LC rate is $86 \%$ (30/34 cavities). The incidence of LMD at 1 year is $12 \%$ (4/34 cavities, 2/4 patients had neuroendocrine NSCLC) with a neurological cause of death in 3 patients. $4 / 44$ patients received SRT and 10/44 had WBRT for new brain metastases/LMD. There has been 1 case of histolgically confirmed RN after immunotherapy.

Conclusions: Our cohort confirms the safety and efficacy of postop hfSRT. The $12 \%$ incidence of LMD approximates postop SRS (7$28 \%$ ) and surgery (16\%), which suggests cavity hfSRT has little impact on disseminated tumour cells in the CSF. Preop SRS is compelling (delineation of intact metastasis, $20 \%$ dose reduction, resection of irradiated volume, sterilisation of disseminated cells) with promising reports of efficacy with minimal toxicity. Preop SRS will be evaluated in a Swiss prospective trial aiming to further improve outcomes after resection of brain metastases.

\section{Tumour progression to the epidural space after spine SBRT and re-SBRT showing tumour pseudoprogression after salvage surgery: A case report \\ Type: Clinical}

Presenting Author:

Emanuel Stutz

Authors:

E. Stutz ${ }^{1}$, M. Wartenberg 2 , DM. Aebersold ${ }^{1}$, E. Herrmann ${ }^{1}$,

H. Hemmatazad ${ }^{1}$

Institutions:

${ }^{1}$ Inselspital Bern, ${ }^{2}$ University of Bern

Department:

${ }^{1}$ Radio-Onkologie, ${ }^{2}$ Pathology

Aim/background: Distinguishing true tumour progression (TTP) from pseudoprogression (PP) in spine stereotactic body radiotherapy (SBRT) is challenging. Based on the SPIne response assessment in Neuro-Oncology (SPINO) group guidelines, one criterion for TTP is defined as unequivocal increase in tumour volume and/or new tumour progression within the epidural space.

We here report a case of a new tumour progression to the epidural space following primary SBRT and re-SBRT with finally salvage vertebrectomy, which allowed an histopathological evaluation of the surgical specimen and radiographic MRI correlation.

Methods: A 78-year old male patient with an anaplastic thyroid carcinoma with an isolated bony metastasis to T-7 was treated with spine SBRT with 24 Gy in 3 fractions based on common delineation guidelines. The MRI 22 months after the first SBRT revealed a new tumour volume progression to the epidural space resulting in a re-SBRT with $30 \mathrm{~Gy}$ in 5 fractions. The 3 and 6 month MRI after re-SBRT showed again a progressing epidural growth (Bilsky 1c). After T-7 vertebrectomy, obtained tissue specimens were histopathologically evaluated.

Results: Only a small cluster of carcinoma cells of $1 \mathrm{~mm}$ diameter was found within a bony structure not belonging to the radiologically highly suspicious epidural mass.

Conclusion: We report the first case of a new tumour progression to the epidural space following primary SBRT and re-SBRT, which histopathologically revealed as PP. Based on the "epidural progression criterion" from the SPINO-guidelines and other predictive factors, this progression should have been classified as TTP. Due to the challenge in distinguishing TTP from PP, reporting of such cases are essential to share experiences and thereby improve the understanding of PP after spine SBRT. 
Fully automated organs at risk delineation for brain tumor radiation planning in patients with glioblastoma using deep learning

Type: Clinical

Presenting Author:

Evelyn Herrmann

Authors:

E. Rüfenacht ${ }^{2}$, A. Jungo ${ }^{2}$, E. Ermiș ${ }^{1}$ H. Hemmatazad ${ }^{1}$, M. BlattiMoreno $^{1}$, D. M. Aebersold ${ }^{1}$, P. Manser ${ }^{3}$, M. K. Fix ${ }^{3}$, M. Reyes ${ }^{2}$ and E. Herrmann ${ }^{1}$

\section{Institutions:}

${ }^{1,3}$ Inselspital, Bern University Hospital and University of Bern Department:

${ }^{1}$ Department of Radiation Oncology, ${ }^{3}$ Division of Medical Radiation Physics, ${ }^{2}$ Institute for Surgical Technology and Biomechanics, Bern

Purpose/objective(s): To evaluate, if fully deep learning (DL) based automated segmentation of organs at risk (OAR) within the brain in glioblastoma (GBM) patients is non-inferior to human rater segmentation for radiation planning.

Materials/methods: We included post-OP MRI datasets of $23 \mathrm{GBM}$ patients. Manual segmentations of OAR were performed by 3 radiation oncologists on T1c MRI sequence. For fully automatic segmentation, we developed an OAR segmentation method. The MRI data was co-registered for automatic segmentation. Manual delineations were utilized for training the DL system based on the U-Net architecture. We evaluated the segmentation method on the results of a 6-fold cross-validation considering the imaging data of all patients in terms of dice-coefficient (DC) (volumetric overlap) and volume similarity (VS) using the Kruskal-Wallis test.

Results: Large distribution of DC and VS were observed for small OAR between the raters $[(0.78,0.12),(0.43,0.13),(0.49,0.12)$; Kruskal-Wallis test: chi-square $=43.80, p=0.001]$ and the automatic method: $[(0.00,0.23),(0.00,0.26),(0.00,0.18)$; Kruskal-Wallis test: chi-square $=81.29, p=0.001]$. For larger OAR, no statistical difference was detected between volumes among raters (Kruskal-Wallis test: chisquare $=4.6, p=0.098)[(0.91,0.02),(0.91,0.02),(0.89,0.02)]$, and compared to the automatic segmentation: $[(0.91,0.02),(0.91,0.01)$, $(0.90,0.02)]$.

Conclusion: Performance of this system is highly dependent on raters agreement. The higher the agreement, the better predicts the prototype the OAR within the brain. With this proof of concept study, we generate first promising results, and plan next steps to improve results.

\section{Safety and efficacy of local irradiation in patients with metastatic melanoma treated with the CTLA-4 antibody ipilimumab}

Type: Clinical

\section{Presenting Author:}

Samantha Weder

Authors:

Samantha Weder ${ }^{1}$, Anna-Lena Hottinger ${ }^{2}$, Sacha Rothschild ${ }^{3}$

\section{Institutions:}

${ }^{1}$ University Hospital Basel, ${ }^{2,3}$ University Hospital Basel

Department:

${ }^{1}$ Radiation Oncology and Medical Oncology, ${ }^{2}$ Department of Radiation Oncology, ${ }^{3}$ Department of Medical Oncology

Introduction: Potentially beneficial interactions of radiotherapy (RT) and the immune checkpoint inhibitor ipilimumab have been reported in patients with melanoma. Here we report on a retrospective study analysing the safety of concurrent radiotherapy with ipilimumab in patients with metastatic melanoma.
Methods: Thirty-two patients with metastatic melanoma treated with ipilimumab and receiving local RT at the University Hospital Basel were included in the trial. Eight Patients received ipilimumab and radiotherapy with a longer time difference than 3 months, irrespective of order of treatment. Because of the long time difference no additive toxicity of the two therapies were expected and these patients were classified as a control group. The radiation-related adverse events of the treatment group $(n=24)$ and control group $(n=8)$ were compared using Fisher's exact test.

Results: The mean cumulative dose of RT was 32.4 Gy (range, 5-62). Twenty-nine patients $(90.6 \%)$ had acute toxicities in the irradiated area (grade 1/2). The statistical analysis using Fisher's exact test showed a significantly lower toxicity rate for "mucositis" $(p=0.037)$ in the treatment group. The patients were irradiated in different areas, which may have caused this surprising result. While the control group had two patients irradiated in a mucositis-associated area, the treatment group only had one patient with RT in a similar area. All other radiation-related adverse events, such as "skin toxicity", "gastrointestinal", "fatigue", "alopecia", "central nervous system" and "others" did not show any significant difference.

Conclusions: The treatment group did not show an increased radiation-related toxicity rate compared to the control group. The toxicity rates for concurrent radiation therapy were not higher than reported in the literature. We therefore conclude that ipilimumab does not substantially increase local side effects of RT.

\section{MR-guided adaptive SBRT for prostate cancer: stability of adaptive planning analyzed by repetitive imaging Type: Clinical}

\section{Presenting Author:}

Jana Heitmann

Authors:

J. Heitmann, M. Chamberlain, M. Baumgartl, J. Krayenbühl,

M. Zamburlini, M. Mayinger, L. Wilke, N. Andratschke,

S. Tanadini-Lang, M. Guckenberger

Institutions:

University Hospital Zurich

Department:

Radiation Oncology

Aims: Magnetic resonance-guided radiotherapy promises reduced toxicity for prostate cancer patients treated with stereotactic body radiotherapy (SBRT) by means of daily plan adaption. However, creation, adaption and quality assurance of a plan-of-the-day takes up to $60 \mathrm{~min}$. To date, the anatomic changes during re-planning are not considered. We therefore performed repeat MR-imaging in healthy volunteers over $60 \mathrm{~min}$ to assess these changes.

Methods: We recruited 10 healthy male volunteers and created baseline SBRT prostate cancer plans for the MRIdian Linear Accelerator (Viewray Inc) of $36.25 \mathrm{~Gy}$ in five fractions. The prostate and the lower $1 / 3$ of the seminal vesicles were delineated as the CTV with a $5 \mathrm{~mm}$ PTV margin ( $3 \mathrm{~mm}$ posteriorly). On a separate day, the original plan was re-planned online using MRI-guidance followed by repeated MR-scans in 15-minute-intervals: changes in dose to OARs and target coverage over time were analysed to investigate the stability of online re-planning.

Results: Here, we report the interim analysis of the first $5 / 10$ volunteers. Online adaptive re-planning improved coverage of the $\mathrm{D}_{95 \%} \mathrm{PTV}_{-}$ Prostate significantly by median of $8 \%(1-28 \% ; p=0.04)$. Based on the repetitive MR-imaging, the $\mathrm{D}_{95 \%} \mathrm{CTV}$ Prostate coverage after $60 \mathrm{~min}$ was significantly reduced by median $8 \%(0-17 \% p=0.04)$, whereas coverage of the adapted plan was not significantly decreased after 45 $\min \left(0-12 \%\right.$ n.s.) or at earlier time points. $D_{1 c c}$ Rectum did not change significantly over $60 \mathrm{~min}$. $\mathrm{D}_{\text {Mean }}$ Bladder decreased significantly with a 
median decrease of $8.53 \mathrm{~Gy}(0.41-10.57 \mathrm{~Gy} ; p=0.02)$ due to bladder filling.

Conclusion: Preliminary data indicate that online adaptive re-planning in prostate SBRT improves target coverage and the benefit of adaptive re-planning is stable for about $45 \mathrm{~min}$. In patients where adaptive re-planning takes longer, careful re-evaluation of the adapted plan before treatment delivery is recommended.

\section{Renal toxicity of total body irradiation with $6 \times 2$ Gy prior to allogenic stem cell transplantation}

Type: Clinical

\section{Presenting Author:}

Jens Lustenberger

Authors:

Jens Lustenberger ${ }^{1}$, Alexandros Papachristofilou ${ }^{1}$, Frank Zimmermann ${ }^{1}$, Alicia Rovo ${ }^{2,3}$, André Tichelli ${ }^{2}$

\section{Institutions:}

${ }^{1,2}$ University Hospital Basel, ${ }^{3}$ University Hospital Bern

Department:

${ }^{1}$ Clinic of Radiation Oncology, ${ }^{2}$ Clinic of Hematology, ${ }^{3}$ Clinic of Hematology

Aim: Assessment of renal insufficiency (RI) after total body irradiation (TBI) with $6 \times 2$ Gy (treatment $2 \times /$ day).

Materials and methods: Data of patients receiving TBI with $6 \times 2$ Gy with a follow-up time of at least 24 months treated until end of 2010 were analyzed, excluding patients with preexisting renal insufficiency. Age, sex and risk factors for RI were assessed (arterial hypertension, hyperlipidemia, diabetes, smoking, obesity, antimycotic drugs, graft versus host disease (GvHD), Ciclosporin A). The effect of TBI on RI was assessed with a case-control-analysis, (TBI vs. chemotherapy-only-conditioning).

Results: 172 patients met all inclusion criteria. RI at 2 years was $14.5 \%$ and $11.6 \%$ at last follow up. In the univariate analysis at last follow-up extensive chronic GvHD, dyslipidemia, arterial hypertension, age over $30 \& 40$ years were significant factors for RI. In the case-control-analysis ( $n=33$ per group) 5 patients in the TBI group $(15.15 \%)$ and 2 patients $(6.06 \%)$ in the non-TBI group had a renal insufficiency after 2 years. At last follow-up, 5 patients $(15.15 \%)$ and 1 patient $(3.03 \%)$ had a renal insufficiency. Statistical analysis did not show any significant results (Kaplan Meier analysis or correlation coefficient).

Discussion: In our retrospective study univariate analysis showed several known conditions as potential factors for RI, but none is found to be leading in multivariate analysis. In the case control analysis TBI was not shown to lead to RI more frequent than chemotherapy-only-conditioning.

Conclusion: Our results match the literature. TBI with $6 \times 2$ Gy does not appear to increase the risk of RI. No individual factor could be identified putting patients at higher risk for RI.

\section{The challenges of radiotherapy in Dakar, Senegal Type: Clinical}

\section{Presenting Author:}

Conny Vrieling

Authors:

Vrieling C, Thengumpallil S, Bezard J.

\section{Institutions:}

Clinique des Grangettes, Geneva

Department:

Radiation Oncology

Aims: Assist the radiotherapy team in Dakar, Senegal, in the transition from $2 \mathrm{D}$ to $3 \mathrm{D}$.
Methods: Early 2018, the radiotherapy department of Hospital le Dantec, Dakar, Senegal installed a linac. In May 2019, a team of a radiation oncologist, medical physicist and dosimetrist from Clinique des Grangettes, Geneva, spent a week in the department to assess the current situation and the training needed in order to optimize patient care. The contact was established through Radiating Hope, www.radiatinghope.org, a non-profit organisation focused on improving radiation oncology care around the globe.

Results: At the end of 2017, the Cobalt machine was replaced by a Varian Unique, a linac with only a $6 \mathrm{MV}$ beam and a MV imager. The team consists of a radiation oncologist and 2 residents, a medical physicist and 2 medical physicist trainees and 6 technicians.

The first patient was treated on 5 March 2018. Overall, 281 patients have been treated in 2018. The average patient age was around 40 years and the majority of tumors were advanced. The most frequent localisation was cervical cancer, followed by ENT and breast cancer.

The installation of the Unique entailed the change from 2D to 3D, which had a great impact on the workflow: CT-scans needed to be contoured followed by dosimetry and during treatment weekly online imaging was performed. The team, while very motivated, was confronted with several challenges. On the medical level, we identified a need for training in contouring. The physicist had acquired equipment for MLC and patient QA, however, missed the support and time to commission. Also, hands-on support in the optimal 3D planning was needed. For the technicians, a standardisation of the procedures at the scanner as well as the machine was requested. We were able to make a start with identified training objectives.

Conclusion: Together with the radiotherapy team in Dakar, Senegal, we were able to identify training needs in the change from $2 \mathrm{D}$ to $3 \mathrm{D}$. We made a first start with and will continue the collaboration with this motivated team that is looking for training.

\section{Systematic review of adverse effect reporting in stage III non- small-cell lung cancer (NSCLC) phase III trials investigating surgery and radiotherapy \\ Type: Clinical}

Presenting Author:

Thomas Iseli

Authors:

Iseli $\mathrm{T}^{1}$, Glatzer $\mathrm{M}^{1}$, Rittmeyer $\mathrm{A}^{2}$, Berghmans $\mathrm{T}^{3}$, Massard $\mathrm{G}^{4}$, Putora $\mathrm{PM}^{1}$

\section{Institutions:}

${ }^{1}$ Kantonsspital St. Gallen, ${ }^{2}$ Lungenfachklinik Immenhausen, ${ }^{3}$ Institut Jules Bordet, Brussels, ${ }^{4}$ University Hospital Strasbourg

Department:

${ }^{1}$ Radio-Onkologie, ${ }^{2}$ Department of Thoracic Oncology, ${ }^{3}$ Department of Intensive Care and Oncological Emergencies \& Thoracic Oncology, ${ }^{4}$ Lung Transplantation Group

Background: When multiple treatments options are available for stage III non-small cell lung cancer patients (NSCLC), risk of adverse events is essential for decision-making.

Objective: We analysed reporting of AEs in phase III radiotherapy or surgery trials for stage III NSCLC.

Methods: Prospective phase III trials for stage III NSCLC (pubmed, 01/2009-04/2019). AE reporting and grading (according CTCAE) were analysed with Chi square and smooth kernel distribution (with bootstrapping).

Results: 5 of 23 trials identified included a surgery. Concurrent radio-chemotherapy was used in 20 trials, 3 used sequential radio-chemotherapy. 18 included only inoperable patients. We identified 159 unique AE items (107 acute, 17 late AEs, 13 surgical, 14 acute and 8 late organ related AEs). Only 28 were included in at least $20 \%$ of the analysed studies (or in more than two for surgical studies). Grade 3 and 4 were reported in $95.65 \%$. Grade 1 and 2 were reported in $34.78 \%$ and 
Table 1 Mean AE/trial

\begin{tabular}{ll}
\hline Toxicity as EP (13) & $15.15 \pm 7.77, p=0.086$ Toxicity not as EP (10): \\
& $25.40 \pm 14.54, p=0.089$ \\
\hline$N<200(11)$ & $13.00 \pm 4.98, p=0.070$ \\
$N \geq 200(12)$ & $25.67 \pm 13.65, p=0.076$ \\
\hline
\end{tabular}

$39.13 \%$ respectively. One study did not grade AEs. 3 out of 4 studies including surgery reported among other AEs surgery-related AEs, but only one graded them. Size and toxicity defined as endpoint (EP) tended to report better, sponsorship had no impact (Table 1)

Conclusion: Overall, the majority reported AE grading and serious AEs. Deaths were reported reliably. Lower grades however, were underreported. Toxicity as an endpoint nor funding source affected reporting frequencies. Under-reporting prohibits the extraction of patient-relevant information for decision making and represents a suboptimal use of invested resources.

\section{Skeletal muscle mass correlates with increased toxicity during neoadjuvant radiochemotherapy in locally advanced esophageal cancer. A SAKK 75/08 substudy \\ Type: Clinical}

Presenting Author:

Dr Panje

Authors:

C. Panje ${ }^{1}$, L. Höng ${ }^{2}$, S. Hayoz ${ }^{3}$, E. Herrmann ${ }^{4}$, H. Garcia Schüler ${ }^{5}$,

U.R. Meier ${ }^{6}$, G. Henke ${ }^{1}$, S. Schacher ${ }^{7}$, M.-A. Gérard ${ }^{3}$, T. Ruhstaller ${ }^{8}$, L. Plasswilm ${ }^{1,9}$ for the Swiss Group for Clinical Cancer Research (SAKK).

\section{Institutions:}

${ }^{1}$ Kantonsspital St. Gallen, ${ }^{2}$ LUKS, ${ }^{3}$ Coordinating Center, Bern, ${ }^{4}$ Inselspital Bern, ${ }^{5}$ University Hospital Zurich, ${ }^{6,7} \mathrm{KSW},{ }^{8} \mathrm{KSSG}$ and

University of Basel, ${ }^{9}$ University of Bern

Department:

${ }^{1}$ Radiation Oncology, ${ }^{2}$ Radiation Oncology, ${ }^{3}$ Swiss Group for Clinical Cancer Research (SAKK), ${ }^{4}$ Radiation Oncology, ${ }^{5}$ Radiation Oncology, ${ }^{6}$ Radiation Oncology, ${ }^{7}$ Medical Oncology, ${ }^{8}$ Medical Oncology and Hematology

Background: The aim of our study was to determine the impact of sarcopenia on prospectively collected specific outcomes in a subgroup of the phase III trial SAKK 75/08 treated with trimodality therapy consisting of induction chemotherapy, neoadjuvant radiochemotherapy and surgery for locally advanced esophageal cancer.

Methods: Sarcopenia was assessed by skeletal muscle index at the third lumbar vertebra (L3) in cross-sectional computed tomography scans before induction chemotherapy, before radiochemotherapy and after neoadjuvant therapy in 61 patients from four centers in Switzerland and correlated with prospectively collected data on toxicity and survival.

Results: The prevalence of sarcopenia defined according to established cut-off values significantly increased from $50.8 \%$ before treatment to $77 \%$ after neoadjuvant therapy. There were non-significant trends towards increased grade $\geq 3$ toxicity during radiochemotherapy ( $71 \%$ vs $51 \%$ ) and postoperative complications in patients with sarcopenia at baseline (58\% vs $41 \%$ ), but trimodality therapy was feasible in $97 \%$ of sarcopenic patients. Using sarcopenia cut-off values specifically for our study population, toxicity differences during neoadjuvant radiochemotherapy became significant ( $78 \%$ vs. $39 \%)$. No difference in survival could be observed.

Conclusion: Trimodality therapy is feasible in selected patients with sarcopenia, but sarcopenic patients are at higher risk for increased toxicity during neoadjuvant radiochemotherapy.
Disclosures: The SAKK 75/08 trial was funded by Merck KGaA, Darmstadt, Germany.

\section{Thermochemoradiotherapy for locally advanced pancreatic cancers: A pilot study \\ Type: Clinical}

Presenting Author:

Dr. med. Emsad Puric

Authors:

Puric E. ${ }^{1}$, Datta N. R. ${ }^{1}$, Timm O. ${ }^{1}$, Marder D. ${ }^{1}$, Mingrone W. ${ }^{2}$, Kralidis E. ${ }^{3}$, Knuchel J. ${ }^{4}$, Hartel M. ${ }^{5}$, Steinauer K. ${ }^{1}$, Riesterer O. ${ }^{1}$, Bodis S. ${ }^{1}$

Institutions:

${ }^{1,3,4,5}$ Kantonsspital Aarau, ${ }^{1}$ Kantonsspital Baden, ${ }^{2}$ Kantonsspital Olten Department:

${ }^{1}$ Centre for Radiation Oncology, ${ }^{2}$ Department of Hematology and Oncology, ${ }^{3}$ Department of Hematology and Oncology, ${ }^{4}$ Department of Gastroenterology and Hepatology, ${ }^{5}$ Department of General and Viszeral Surgery

Introduction/aim: Thus a pilot study was initiated to explore the safety and efficacy of concurrent thermochemoradiotherapy (HTCTRT) in LAPC with neoadjuvant and post-HTCTRT FOLFIRINOX.

Methods: A total of 8 patients (mean age: 65 years) were evaluated in this study. In 7, the T stage was T3 or T4/N1 disease, 2 patients were M1. All received 4 cycles of neoadjuvant chemotherapy (NACT) with FOLFIRINOX followed by HTCTRT. The HTCTRT consisted of concurrent gemcitabine $\left(400 \mathrm{mg} / \mathrm{m}^{2}\right.$ on D1, weekly) followed by simultaneous boost intensity modulated radiotherapy (SIB-IMRT) delivering $56 \mathrm{~Gy} / 28$ fractions to the gross tumor and lymph nodes over 5.5 weeks. Deep regional hyperthermia (HT, total 4 to 6 sessions) was administered weekly on D2 prior to SIB-IMRT. It was monitored with real-time intraduodenal multisensor thermometry at each session (max. temperature of $40.8{ }^{\circ} \mathrm{C} / \max$. power of $750 \mathrm{~W}$ ). Following HTCTRT, a median of 8 cycles of FOLFIRINOX was delivered.

Results: Follow-up ranged from 3 to 19 months (median: 10 months). Three patients have a complete response on PET-CT while in 2, the disease was stable. Six patients are alive to date. The median progression free (PFS) and overall survival (OS) were 19 and 15 months respectively. Correspondingly, 1 year PFS and OS was $60 \%$ and $80 \%$. None of the patients had any grade III/IV acute or late toxicities, overall compliance was satisfactory.

Conclusion: HTCTRT in LAPC appears to be a viable therapeutic option. It is well tolerated, safe with encouraging outcomes. This merits further evaluation in a open multi-centric clinical study (HEATPAC).

\section{NRG international membership - a launchpad for international randomised trials}

Type: Clinical

Presenting Author:

Susanne Rogers

Authors:

Rogers $\mathrm{S}^{1}$, Khan $\mathrm{S}^{1}$, Corn $\mathrm{B}^{2}$, Bodis $\mathrm{S}^{1}$

Institutions:

${ }^{1}$ Kantonsspital Aarau, Kantonsspital Baden, ${ }^{2} \mathrm{Tel}$ Aviv University

Department:

${ }^{1}$ Radiation Oncology Center, ${ }^{2}$ Radiation Oncology

Aim: In response to a call for initiatives from NRG Oncology (NSABP, RTOG and GOG) international members, Kantonsspital Aarau has proposed a trial to determine the optimal radiation therapy for patients with 5 or more melanoma brain metastases (BM). We describe the process of developing an international research protocol within this framework. 
Method: In the absence of patients with melanoma in the observational study by Yamamoto et al. [1]-where non-inferior overall survival was reported following SRS for 5-10 BM as compared with 1-4 BM-we conceived a trial randomizing between SRS and neurocognitive-sparing WBRT in patients with 5 or more melanoma BMs.

Results: An overview was presented at the NRG Semiannual Meeting in February 2019. Feedback was positive and at a follow-up conference call with a US radiation oncologist interested in being the required "study champion" and undertaking the translational research, a decision was taken to submit a 10 page study concept to the NRG Brain Committee. The latter has accepted the protocol for discussion at the NRG Research Meeting in July 2019.

Conclusions: The further progress of the trial depends on a possible juxtaposition with a competing study concept, however in 3 months, our "back of the envelope" idea has acquired the potential to be adopted as an NRG phase III trial protocol open to recruitment worldwide.

\section{References}

1. Yamamoto M, Serizawa T, Shuto T et al (2014) ,Stererotactic Radiotherapy for patients with multiple brain metastases (JLGK0901) a multi-institutional prospective observational study ', Lancet Oncology 15(4):387-95

\section{Extremely hypofractionated salvage radiotherapy for isolated local recurrent prostate cancer: toxicity and biochemical control \\ Type: Clinical}

\section{Presenting Author:}

Nicolas Bachmann

Authors:

Nicolas Bachmann, Elena Riggenbach, Olgun Elicin, Mohamed

Shelan

\section{Institutions:}

Inselspital Bern, Bern University Hospital and University of Bern

Department:

Department of Radiation Oncology

Aims: Biochemical failure due to isolated local recurrence (LR) after primary treatment is a common problem in prostate cancer (PC). Extremely hypofractionated salvage Radiotherapy (ehSRT) could be a valuable therapy option in this setting. We aimed to evaluate the safety and efficacy of (ehSRT) in patients with isolated LR after definitive radiotherapy (RT) or radical prostatectomy (RP).

Methods: Between 2015 and 2019, 12 patients received ehSRT due to isolated LR after primary treatment. Isolated LRs initially and after ehSRT were diagnosed through pelvic magnetic resonance imaging (MRI) and positron emission tomography (PET). A total dose of 35 Gy in five fractions was delivered every second day. No androgen deprivation therapy was given. Baseline, acute and chronic gastrointestinal (GI) and genitourinary $(\mathrm{GU})$ toxicities were assessed according to CTCAE v.4.

Results: Median time from primary treatment to ehSRT was 74 months (19.5-116.8 months). Median PSA value before ehSRT was $4.7 \mathrm{ng} / \mathrm{ml}$ $(0.2-14.6 \mathrm{ng} / \mathrm{ml})$. On a median follow-up time of 16.6 months $(4-41.8$ months), 7 (58\%) patients had evidence of biochemical progression. Out of these patients, 3 (25\%) experienced LR, 1 (8\%) distant metastasis (DM) and $3(25 \%)$ both LR and DM. Prior to ehSRT, 4 (33\%) patients suffered from grade 1 chronic urinary toxicity. Up to 3 months after ehSRT, $1(8 \%)$ patient developed grade 1 and $2(17 \%)$ patients developed grade 2 acute GU toxicity. On a median follow up of 16.6 months, only $1(8 \%)$ patient suffered from grade 1 chronic GU disorder and another patient developed grade 1 chronic GI disorder. No grade 3 or 4 acute or chronic GU or GI toxicities were observed.

Conclusion: In this cohort, no high-grade relevant acute or chronic toxicity was observed after ehSRT to isolated LR. Reasonable biochemical control rates were achieved. Long-term follow-up is needed.
Oldest old: Challenges and limitations in radiotherapy for the elderly

Type: Clinical

Presenting Author:

Eva-Maria Kretschmer

Institutions:

University Hospital Zurich

Department:

Department of Radiation Oncology

Purpose: Older patients frequently suffer from multiple chronic diseases and have a high risk of being categorized as frail. Little is known about rational radiation concepts for this particular patient group. The aim of this study was to investigate outcome and clinical efficacy of radiation therapy in a group of oldest old cancer patients ( $\geq 85$ years). Materials and methods: We performed a retrospective analysis of data from patients aged $\geq 85$ years treated for any primary tumor site at the University Hospital Zurich from 2010 to 2015. We assessed overall survival (OS), progression-free survival (PFS), performance status (ECOG), Charlson-comorbidity index (CCI) and, treatment tolerance analog common terminology criteria for adverse events (CTCAE).

Results: Our study included 102 patients with a mean age of 87 years (range: 85-102 y). Most patients received a curative intended treatment $(n=66[64.7 \%])$ with a pre-radiotherapy ECOG of $0-1$ in $84 \%$ of the cases, followed by palliative radiation therapy for metastasis $(n=36[35.3 \%])$, with a pre-radiotherapy ECOG $0-1$ in $58,3 \%$ and $2-3$ in $52,7 \%$. Head and neck cancer $(n=18$ [28.6\%]), breast cancer $(n=13$ [20.6\%]), and skin cancer $(n=12[19.0 \%])$ were the most common cancer types in curative treatment. Four patients $(2.5 \%)$ did not complete radiotherapy, and 10 treatment courses $(6.3 \%)$ were interrupted. $\mathrm{Cu}-$ rative treatment was tolerated with no severe or high-grade toxicities (4+). Median OS was 23 months (range 0-89 m), PFS was 19 months (range $0-89 \mathrm{~m}$ ).

Discussion: In an ageing population, the number of old and oldest old cancer patients will increase considerably in the next decades. So far, there have been no specific treatment guidelines for the management of this specific population. It is crucial to consider co-morbidities and functional as well as social status in the treatment concept. Our analysis supports the notion that radiation therapy in oldest old cancer patients is feasible and well tolerated. It should therefore be validated in prospective studies.

\section{First MRI guided cardiac radioablation of sustained ventricular} tachycardia

Type: Clinical

\section{Presenting Author:}

Michael Mayinger

Authors:

Mayinger $\mathrm{M}^{* 1}$, Kovacs $\mathrm{B}^{* 2}$, Tanadini-Lang $\mathrm{S}^{1}$, Ehrbar $\mathrm{S}^{1}$,

Wilke $\mathrm{L}^{1}$, Brunckhorst $\mathrm{C}^{2}$, Duru $\mathrm{F}^{2}$, Steffel $\mathrm{J}^{2}$, Breitenstein $\mathrm{A}^{2}$,

Alkadhi $\mathrm{H}^{3}$, Garcia Schueler $\mathrm{HI}^{1}$, Ruschitzka $\mathrm{F}^{2}$, Guckenberger $\mathrm{M}^{1}$, Saguner $\mathrm{AM}^{* 2}$, Andratschke N*1

*Shared first/last authors

Institutions:

University Hospital Zurich

Department:

${ }^{1}$ Department of Radiation Oncology, ${ }^{2}$ University Heart Center Zurich, ${ }^{3}$ Institute of Diagnostic and Interventional Radiology

Aim: We report the first magnetic resonance image guided radioablation (MR-IGRA) in a patient with dilated cardiomyopathy and recurrent sustained ventricular tachycardia (VT) associated with several electrical storms. 
Methods: A 71-year-old male patient with non-ischemic dilated cardiomyopathy continued suffering from recurrent life-threatening VT and electrical storms with recurrent antitachycardia pacing and ICD shocks, despite optimized guideline-directed heart failure treatment, maximal antiarrhythmic medication, two endocardial radiofrequency catheter and one epicardial surgical ablation.

Results: Phantom measurements showed a distortion of less than $1.5 \mathrm{~mm}$ due to the ICD. Areas of anatomical scarring and critical substrate in the basal LV were identified to build a volumetric target using invasive three-dimensional electroanatomical mapping of the VT during previous electrophysiologic studies as well as cardiac MRI/CT. The patient was treated with a single fraction of 25 Gy @ 85\% isodose. Cine tracking time was $46 \mathrm{~min}$, beam on time $24 \mathrm{~min}$. $24 \mathrm{~h}$ post intervention the patients developed aggravation of $\mathrm{VT}$ and a prolonged electrical storm. VT ceased following high-dose dexamethasone administration at day 2 after MR-IGRA. In the first month of follow-up no episodes of VT and complications were detected. Left ventricular ejection fraction remained stable at $25 \%$.

Conclusion: In this first MR-IGRA, real-time tracking and beam gating was successfully applied to treat a patient with dilated cardiomyopathy and recurrent therapy-refractory sustained VT with electrical storms. As of two days post treatment the patient has been without episodes of sustained tachyarrhythmia.

\section{Head and neck cancer of the elderly: tumor, patient characteristics and patterns of care in a tertiary cancer center Type: Clinical}

Presenting Author:

Jean-Jacques Stelmes

Authors:

Stelmes J-J, Schanne D, Sandner U, Aleksandrowicz A, Broglie Däppen M.A, Schumann P, Stahel R, Guckenberger M, Balermpas P

\section{Institutions:}

University Hospital Zurich

Department:

Radiation-Oncology

Aims: With the continuous increase of the ageing population and the rising incidence of head and neck cancer (HNC) the need to generate real-world evidence for elderly patients (pts) becomes more urgent than ever. Here we present data regarding epidemiology and treatment of elderly ( $65 \mathrm{y})$ pts with HNC.

Methods: We retrospectively analyzed the OnkoStar ${ }^{\circledR}$ database of the Comprehensive Cancer Center Zurich (CCCZ). Between 2013 and 2018, 792 pts were divided into two major groups: 1. Pts with $65 \mathrm{y}$ (elderly) and 2. Pts $<65 \mathrm{y}$. Elderly were than divided according to the WHO in 3 subgroups (old, older old, oldest old). We performed descriptive and comparative analyses of clinicopathological characteristics using pearson's chi-squared test and finally also tumor-control (TC) and survival analyses (OS) implementing Kaplan-meier curves and cox regression models.

Results: $52 \%(n=410)$ pts. with HNC were classified as "elderly". The most common diagnosis in younger pts was cancer of the oral cavity $(35.6 \% ; 32.9 \%$ for young vs. elderly) and in elderly oropharynx $(31.4 \%$ vs. $36.6 \%)$. Tumor stage was similar $\left(\chi^{2}=0.864 ; p=0.834\right)$. Elderly received just as many curative intent regimens like combined surgery and irradiation $(12.2 \%$ vs $10.6 \%, p=0.489)$ but less concomitant radio-chemotherapy (RCT) $(24.7 \%$ vs. $17.9 \%, p=0.021)$ and this was also the case with rising age $(p=0.002)$. The most common treatment modality used for elderly was surgery alone $(55.0 \%)$. The frequency of Cetuximab did not differ $(p=0.332)$ as $3 y$ TC $(77.4 \%$ vs. $77.9 \%, p=0.556$ ) but elderly showed lower rates of $2 \mathrm{y}$ OS: $72.5 \%$ vs $59.4 \%, p<0.001$. In UICC III-IV stages treated with primary $\mathrm{R}(\mathrm{CT})$ compared to other treatment strategies, OS was identical in both groups $(p=0.352)$.
Conclusion: There exist some important differences in treatment strategies and outcome between younger and elderly (65 y) with HNC, although high TC rates were achievable.

\section{Chabner XRT CIVCO Bra: clinical application at KSGR Type: Clinical}

Presenting Author:

Sandro Taccogna

Institutions:

Kantonsspital Graubünden

Department:

Radio-Onkologie

The Chabner RT Bra is a radiotherapy positioning aid specifically targeted to patients that present with pendulous breasts. The aim of this bra is to reduce skin reactions inferior to the mammory fold, reduce lateral breast sag and in some ways provide an alternate to prone breast radiotherapy. In addition, the Chabner Bra provides dignity to the patient, increases stablitiy to breast positioning and aids in reproducibility during treatment. This presentation outlines our clinical findings at Kantonsspital Gräubunden (KSGR) Radio-Onkologie. This includes documentation of the process from CT/RT Planning/Treatment, as well as skin reactions and feedback from the KSGR staff and patients.

\section{Stereotactic body radiotherapy in early stage non-small cell} lung cancer: mono-institutional experience

Type: Clinical

Presenting Author:

Borgonovo Giulia

Authors:

Borgonovo G, Martucci F, Cima S, Richetti A.

Institutions:

Oncology Institute of Southern Switzerland, Bellinzona-Lugano

Department:

Radiation Oncology Clinic

Aim: Stereotactic body radiotherapy (SBRT) offers local control and cancer outcomes approaching surgical resection with lower risk of treatment-related morbidity, making SBRT the treatment of choice for medically inoperable patients with early-stage non-small-cell lung cancer (NSCLC). The aim of this study was to report the clinical outcomes in our institutional experience of patients treated with SBRT in the definitive management of NSCLC.

Methods: We analysed 35 patients with medically inoperable stage I NSCLC (squamous cell carcinoma and adenocarcinoma) who were treated in our institution with curative intent from September 2011 to February 2018. All patients were treated with SBRT with VMAT/RapidArc technique, up to a curative dose. The SBRT schedules included 50 Gy in 5 fractions $(\mathrm{BED}=100$ considering $\alpha / \beta 10)$, or 54 Gy in 3 fractions ( $B E D=151$ considering $\alpha / \beta 10)$, at the $95 \%$ isodose of the planning target volume. Response was assessed in a 3 monthly follow up. Overall survival (OS) was defined as the time from the end of radiation therapy to death from any cause. Progression-free survival (PFS) was defined as the time from the end of radiation therapy to the first loco regional recurrence and/or metastases. Survival curves/outcomes were calculated using Kaplan-Meier method.

Results: The median age of the patients was 75 years (range $60-90$ years). The mean follow-up was 18 months. The 1-year and 2-year PFS was $87.2 \%$ and $67.2 \%$, respectively. Overall survival at 1 and 2 year was $83.5 \%$ and $64.1 \%$, respectively.

Conclusion: In this study of our early-stage NSCLC patients, we highlight the extremely high efficacy of definitive intent SBRT with a oneyear PFS rate of $87.2 \%$. 